\title{
THE THEORY OF CMB ANISOTROPIES
}

\author{
R. Durrer \\ Université de Genève, Département de Physique Théorique, Quai E. Ansermet 24, 1211 Genève 4, Switzerland; \\ School of Natural Sciences, Institute for Advanced Study Einstein Drive, Princeton, NJ 08540, USA
}

(Received September 24, 2001)

This is a review of the theory of cosmic microwave background (CMB) anisotropies. It is an updated version of a course given in spring 1999 at the 'troisieme cycle de la Suisse Romande'. An introduction to gauge invariant cosmological perturbation theory is given and CMB anisotropies are treated in this context. Simple analytical approximations for the acoustic peak positions for adiabatic and isocurvature perturbations are derived. Silk damping is discussed by an analytic approximation. A short description of the present status of observations and parameter estimation followed by a critical discussion terminated the review. The full system of differential equations for CMB anisotropies and polarization needed in a numerical treatment is also developed and given in an appendix.

Key words: cosmic microwave background, anisotropies, Sachs Wolfe effect, acoustic peaks, Silk damping.

PACS number(s): 98.80 98.80.Es $98.80 . \mathrm{Hw} 98.80 . \mathrm{Cq}$

\section{CONTENTS}

I Introduction

177

A Friedmann-Lemaître universes . . . . . 178

B Recombination and the cosmic microwave background (CMB) . . . . 180

II Perturbation Theory

181

A Gauge transformation, gauge invariance 182

B Gauge invariant perturbation variables 183

1 Metric perturbations . . . . . . . . 183

2 Perturbations of the energy momentum tensor . . . . . . . . . 184

C Basic perturbation equations . . . . . 185

1 Constraint equations . . . . . . . 185

2 Dynamical equations . . . . . . . . 185

3 Conservation equations . . . . . . 186

III Simple applications

186

A The pure dust fluid at $\kappa=0, \Lambda=0$. 186

B The pure radiation fluid, $\kappa=0, \Lambda=0.187$

$\mathrm{C}$ Adiabatic and isocurvature initial conditions for a matter \& radiation fluid . 187

1 Adiabatic initial conditions . . . . . 188

2 Isocurvature initial conditions . . . 188

3 Vector perturbations of perfect fluids 190

4 Tensor perturbations ....... 190

IV CMB anisotropies

A Light-like geodesics . . . . . . . . . 190

B Power spectra . . . . . . . . . . . 192

C The Boltzmann equation ....... 196

1 Elements of the derivation . . . . . 196

2 The tight coupling limit . . . . . . . 198

3 Damping by photon diffusion . . . . 199

D Polarization and moment expansion . 200

E Parameter estimation . . . . . . . . 202

1 Physical processes . . . . . . . . 202
2 Scale dependence . . . . . . . . . 202

3 The main influence of cosmological parameters ... . . . . . . . 202

4 Degeneracy ........... . . 203

V Observations and Results

\section{Appendix A: The $C_{\ell}$ 's from gravitatio-} nal waves

\section{INTRODUCTION}

In this review I would like to show the importance and the power of measurements of anisotropies in the CMB.

CMB anisotropies are so useful mainly because they are small: For a given model, they can be calculated within linear perturbation theory, to very good approximation. They are influenced only little by the non-linear processes of galaxy formation. This allows us to compute them very precisely (to about $1 \%$, which is high precision for present cosmological standards). For given initial fluctuations, the result depends only on the cosmological parameters. If we can measure CMB anisotropies to a precision of, say $1 \%$, this allows us therefore to determine cosmological parameters to about $1 \%$. An unprecedented possibility! Consider that at present, after the work of two generations, e.g., the Hubble parameter is known only to about $25 \%$, the baryon density is known to about $10 \%$ and the uncertainties in the dark matter density, the cosmological constant and the space curvature are even larger. 


\section{R. DURRER}

This somewhat too optimistic conclusion has however three caveats which we want to mention before entering the subject of this review.

1. Initial conditions: The result depends on the model for the initial fluctuations. The simplest inflationary scenarios which lead to adiabatic perturbations, contain in general three to four free parameters, like the ratio of tensor to scalar perturbations $(r)$ and the spectral index of the scalar and tensor perturbations ( $n_{S}$ and $n_{T}$ ), so a few more parameters need to be fitted additionally to the data.

More generic initial conditions allow for at least four additional isocurvature modes with arbitrary (anti-)correlations. The initial conditions are then given by a $5 \times 5$ positive semi-definite matrix, and, in principle, several spectral indices $[1,2]$. In most of this review we shall ignore this possibility and assume that initial perturbations are purely adiabatic. Even if isocurvature constributions cannot be excluded, this most simple model is in good agreement with the present data.

If the perturbations are generated by active sources like, e.g., topological defects, then the modeling is far more complicated, and the analysis is too different to be included in this review.

2. Degeneracy: Even though we can measure over 1000 independent modes ( $C_{\ell}$ 's) of the CMB anisotropy spectrum, there are certain combinations of the cosmological parameters that lead to degeneracies in the CMB spectrum. The result is, e.g., very sensitive to the sum $\Omega_{\text {matter }}+\Omega_{\Lambda}$, but not to the difference ("cosmic confusion").

3. Cosmic variance: Since the fluctuations are created by random processes, we can only calculate expectation values. Yet we have only one universe to take measurements ("cosmic variance"). For smallscale fluctuations we can in general assume that the expectation value over ensembles of universes is the same as a spatial average (a kind of ergodic hypothesis), but for large scales we cannot escape large statistical errors.

\section{A. Friedmann-Lemaître universes}

Friedmann-Lemaitre universes are homogeneous and isotropic solutions of Einstein's equations. The hypersurfaces of constant time are homogeneous and isotropic, i.e., spaces of constant curvature with metric $a^{2}(\eta) \gamma_{i j} d x^{i} d x^{j}$, where $\gamma_{i j}$ is the metric of a space with constant curvature $\kappa$. This metric can be expressed in the form

$$
\begin{gathered}
\gamma_{i j} d x^{i} d x^{j}=d r^{2}+\chi^{2}(r)\left(d \vartheta^{2}+\sin ^{2} \vartheta d \varphi^{2}\right), \\
\chi^{2}(r)= \begin{cases}r^{2} & , \kappa=0 \\
\sin ^{2} r & , \kappa=1 \\
\sinh ^{2} r & , \kappa=-1,\end{cases}
\end{gathered}
$$

where we have rescaled $a(\eta)$ such that $\kappa= \pm 1$ or 0 . (With this normalization the scale factor $a$ has the dimension of a length and $\eta$ and $r$ are dimensionless for $\kappa \neq 0$.) The four-dimensional metric is then of the form

$$
g_{\mu \nu} d x^{\mu} d x^{\nu}=-a^{2}(\eta) d \eta^{2}+a^{2}(\eta) \gamma_{i j} d x^{i} d x^{j} .
$$

Here $\eta$ is called the conformal time.

Einstein's equations reduce to ordinary differential equations for the function $a(\eta)$ (with $\equiv d / d \eta$ ):

$$
\begin{aligned}
\left(\frac{\dot{a}}{a}\right)^{2} & +\kappa=\frac{8 \pi G}{3} a^{2} \rho+\frac{1}{3} \Lambda a^{2} \\
\left(\frac{\dot{a}}{a}\right)^{\prime} & =-\frac{4 \pi G}{3} a^{2}(\rho+3 p)+\frac{1}{3} \Lambda a^{2} \\
& =\left(\frac{\ddot{a}}{a}\right)-\left(\frac{\dot{a}}{a}\right)^{2},
\end{aligned}
$$

where $\rho=-T_{0}^{0}, p=T_{i}^{i}$ (no sum!) and all other components of the energy momentum tensor have to vanish by the requirement of isotropy and homogeneity. $\Lambda$ is the cosmological constant.

Energy momentum "conservation" (which is also a consequence of (1.4) and (1.5) due to the contracted Bianchi identity) reads

$$
\dot{\rho}=-3\left(\frac{\dot{a}}{a}\right)(\rho+p) .
$$

After these preliminaries (which we suppose to be known to the audience) let us answer the following question: Given an object with comoving diameter $\lambda$ (or physical size $a(\eta) \lambda=d)$ at a redshift $z(\eta)=\left(a_{0} / a\right)-1$. Under which angle $\vartheta(\lambda, z)$ do we see this object today and how does this angle depend on $\Omega_{\Lambda}$ and $\Omega_{\kappa}$ ?

We define

$$
\begin{aligned}
& \Omega_{m}=\left(\frac{8 \pi G \rho a^{2}}{3\left(\frac{\dot{a}}{a}\right)^{2}}\right)_{\eta=\eta_{0}}, \\
& \Omega_{\Lambda}=\left.\frac{\Lambda a^{2}}{3\left(\frac{\dot{a}}{a}\right)^{2}}\right|_{\eta=\eta_{0}}, \\
& \Omega_{\kappa}=\left.\frac{-\kappa}{\left(\frac{\dot{a}}{a}\right)^{2}}\right|_{\eta=\eta_{0}},
\end{aligned}
$$

where the index 0 indicates the value of a given variable today. Friedmann's equation (1.4) then requires

$$
1=\Omega_{m}+\Omega_{\Lambda}+\Omega_{\kappa} \text {. }
$$

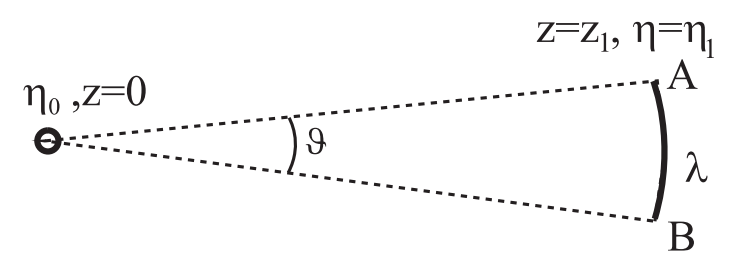

Fig. 1. The two ends of the object emit a flash simultaneously from $A$ and $B$ at $z_{1}$ which reaches us today. 
Back to our problem: Without loss of generality we set $r=0$ at our position and thus $r=r_{1}=\eta_{0}-\eta_{1}$ at the position of the flashes, $A$ and $B$ at redshift $z_{1}$. If $\lambda$ denotes the comoving arc length between $A$ and $B$ we have $\lambda=\chi\left(r_{1}\right) \vartheta=\chi\left(\eta_{0}-\eta_{1}\right) \vartheta$, i.e.,

$$
\vartheta=\frac{\lambda}{\chi\left(\eta_{0}-\eta_{1}\right)} \text {. }
$$

It remains to calculate $\left(\eta_{0}-\eta_{1}\right)\left(z_{1}\right)$.

Note that in the case $\kappa=0$ we can still normalize the scale factor $a$ as we want, and it is convenient to choose $a_{0}=1$, so that comoving scales today become physical scales. However, for $\kappa \neq 0$, we have already normalized $a$ such that $\kappa= \pm 1$ and $\chi=\sin r$ or $\sinh r$. We have in principle no normalization constant left.

From the Friedmann equation we have

$$
\dot{a}^{2}=\frac{8 \pi G}{3} a^{4} \rho+\frac{1}{3} \Lambda a^{4}-\kappa a^{2} .
$$

We assume that $\rho$ is a combination of "dust" (cold, non-relativistic matter) with $p_{d}=0$ and radiation with $p_{\mathrm{rad}}=1 / 3 \rho_{\mathrm{rad}}$.

From (1.6) we find that $\rho_{\text {rad }} \propto a^{-4}$ and $\rho_{d} \propto a^{-3}$. Therefore, with $H_{0}=\left(\frac{\dot{a}}{a^{2}}\right)\left(\eta_{0}\right)$, we define

$$
\begin{aligned}
& \frac{8 \pi G}{3} a^{4} \rho=H_{0}^{2}\left(a_{0}^{4} \Omega_{\mathrm{rad}}+\Omega_{d} a a_{0}^{3}\right) \\
& \frac{1}{3} \Lambda a^{4}=H_{0}^{2} \Omega_{\Lambda} a^{4} \\
& -\kappa a^{2}=H_{0}^{2} \Omega_{\kappa} a^{2} a_{0}^{2} .
\end{aligned}
$$

The Friedmann equation then implies

$$
\frac{d a}{d \eta}=H_{0} a_{0}^{2}\left(\Omega_{\mathrm{rad}}+\frac{a}{a_{0}} \Omega_{d}+\frac{a^{4}}{a_{0}^{4}} \Omega_{\Lambda}+\frac{a^{2}}{a_{0}^{2}} \Omega_{\kappa}\right)^{\frac{1}{2}}
$$

so that

$$
\begin{aligned}
& \eta_{0}-\eta_{1}=\frac{1}{H_{0} a_{0}} \\
& \times \int_{0}^{z_{1}} \frac{d z}{\left[\Omega_{\mathrm{rad}}(z+1)^{4}+\Omega_{d}(z+1)^{3}+\Omega_{\Lambda}+\Omega_{\kappa}(z+1)^{2}\right]^{\frac{1}{2}}} .
\end{aligned}
$$

Here we have introduced the cosmological redshift $z+1=$ $a_{0} / a$. (In principle we could of course also add other matter components like, e.g., "quintessence" [9], which would lead to a somewhat different form of the integral (1.15), but for definiteness, we remain with dust, radiation and a cosmological constant.)

In general, this integral has to be solved numerically. It determines the angle $\vartheta\left(\lambda, z_{1}\right)$ under which an object with comoving size $\lambda$ at $z_{1}$ is seen.

On the other hand, the angular diameter distance to an object of physical size $d$ seen under angle $\vartheta$ is given by $\eta_{0}-\eta_{1}=r_{1}=\chi^{-1}\left(\frac{d}{a_{1} \vartheta}\right)$. If we are able to measure the redshift and the comoving angular diameter distance of a certain class of objects comparing with Eq. (1.15) allows in principle to determine the parameters $\Omega_{m}, \Omega_{\Lambda}$, $\Omega_{\kappa}$ and $H_{0}$.
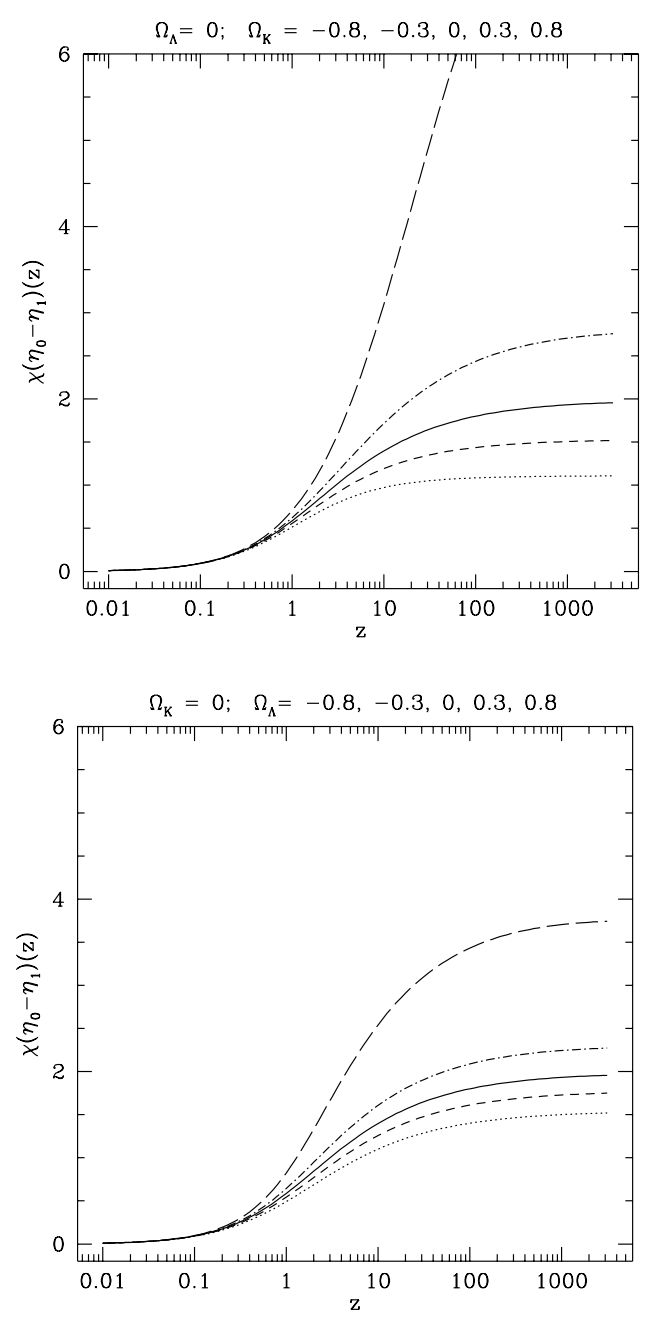

Fig. 2. The function $\chi\left(\eta_{0}-\eta_{1}\right)$ as a function of the redshift $z$ for different values of the cosmological parameters $\Omega_{\kappa}$ (top, with $\Omega_{\Lambda}=0$ ) and $\Omega_{\Lambda}$ (bottom, with $\Omega_{\kappa}=0$ ), namely -0.8 [dotted], -0.3 [short-dashed], 0 [solid], 0.3 [dot-dashed], 0.8 [long-dashed].

We have $\frac{-\kappa}{H_{0}^{2} a_{0}^{2}}=\Omega_{\kappa} \Rightarrow H_{0} a_{0}=\frac{1}{\sqrt{\left|\Omega_{\kappa}\right|}}$ for $\Omega_{\kappa} \neq 0$.

Observationally we know $10^{-5}<\Omega_{\mathrm{rad}} \leq 10^{-4}$ as well as $0.1 \leq \Omega_{d} \lesssim 1,\left|\Omega_{\Lambda}\right| \lesssim 1$ and $\left|\Omega_{\kappa}\right| \lesssim 1$.

If we are interested in small redshifts, $z_{1} \lesssim 10$, we may safely neglect $\Omega_{\mathrm{rad}}$. In this region, Eq. (1.15) is very sensitive to $\Omega_{\Lambda}$ and provides an excellent mean to constrain the cosmological constant.

At high redshift, $z_{1} \gtrsim 1000$, neglecting radiation is no longer a good approximation.

We shall later need the opening angle of the horizon distance,

$$
\begin{aligned}
& \vartheta_{H}\left(z_{1}\right)=\frac{\eta_{1}}{\chi\left(\eta_{0}-\eta_{1}\right)}, \\
& \eta_{1}=\frac{1}{H_{0} a_{0}} \\
& \times \int_{z_{1}}^{\infty} \frac{d z}{\left[\Omega_{\mathrm{rad}}(z+1)^{4}+\Omega_{d}(z+1)^{3}+\Omega_{\Lambda}+\Omega_{\kappa}(z+1)^{2}\right]^{\frac{1}{2}}} .
\end{aligned}
$$




\section{R. DURRER}

(Clearly this integral diverges if $\Omega_{\mathrm{rad}}=\Omega_{d}=0$. This is exactly what happens during an inflationary period and leads there to the solution of the horizon problem.)
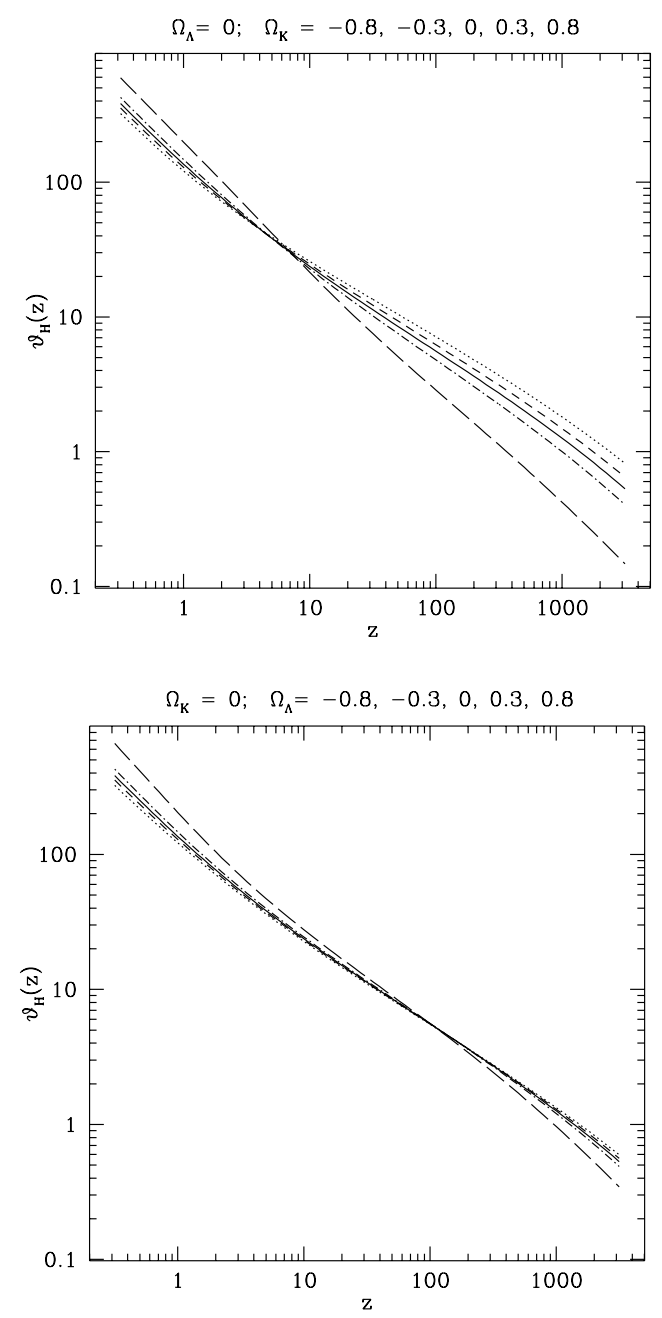

Fig. 3. $\vartheta_{H}\left(z_{1}\right)$ (in degrees) for different values of the cosmological parameters $\Omega_{\kappa}$ and $\Omega_{\Lambda}$ the line styles are as in Fig. 2.

The value of the radiation density is well known. For photons plus three sorts of massless neutrinos we have

$$
\rho_{\mathrm{rad}}=7.94 \times 10^{-34}\left(T_{0} / 2.737 \mathrm{~K}\right)^{4} \mathrm{~g} / \mathrm{cm}^{3} .
$$

This gives

$$
\begin{aligned}
& \Omega_{\mathrm{rad}} h^{2}=4.2 \cdot 10^{-5}\left(T_{0} / 2.737 \mathrm{~K}\right)^{4} \\
& H_{0}=100 h \frac{\mathrm{km}}{\mathrm{s} \cdot \mathrm{Mpc}}
\end{aligned}
$$

Neglecting $\Omega_{\mathrm{rad}}$, for $\Omega_{\Lambda}=0$ and small curvature, $0<\left|\Omega_{\kappa}\right| \ll \Omega_{d}$ at high enough redshift, $z_{1} \geq 10$, one has $\eta_{0}-\eta_{1} \simeq 2 \sqrt{\left|\Omega_{\kappa}\right| / \Omega_{d}}=2 /\left(H_{0} a_{0} \sqrt{\Omega_{d}}\right)$. This yields $\vartheta\left(\lambda, z_{1}\right) \simeq \sqrt{\Omega_{d}} H_{0} a_{0} \lambda / 2=\frac{1}{2} \sqrt{\Omega_{d}} H_{0} \lambda_{\text {phys }} /\left(z_{1}+1\right)$, where $\lambda_{\text {phys }}=a_{1} \lambda$ is the physical scale corresponding to comoving size $\lambda$.

\section{B. Recombination and the cosmic microwave background (CMB)}

During its expansion, the universe cools adiabatically. At early times, it is dominated by a thermal radiation background with $\rho=C / a^{4}=g_{\text {eff }} a_{\mathrm{SB}} T^{4}[10]$, and we find that $T \propto a^{-1}$. Here $g_{\text {eff }}=n_{b}+7 / 8 n_{F}$ is the effective number of degrees of freedom, bosons counting as 1 and fermions counting as $7 / 8$ (see, e.g., [14]). At temperatures below $0.5 \mathrm{MeV}$ only neutrinos and photons are still relativistic leading to the density parameter given in Eq. (1.18). (Neutrinos have a somewhat lower temperature than photons, $T_{\nu}=(4 / 11)^{1 / 3} T$, since they have already dropped out of thermal equilibrium at $T \simeq 1 \mathrm{MeV}$, before $e^{ \pm}$annihilation which therefore reheats the photons but not the neutrinos, see, e.g., $[14,15]$.)

The photons obey a Planck distribution,

$$
f(\omega)=\frac{1}{e^{\omega / T}-1} .
$$

At a temperature of about $T \sim 4000 \mathrm{~K} \sim 0.4 \mathrm{eV}$, the number density of photons with energies above the hydrogen ionization energy drops below the baryon density of the universe, and the protons begin to (re-)combine to neutral hydrogen. (Helium has already recombined earlier.) Photons and baryons are tightly coupled before (re-) combination by non-relativistic Thomson scattering of electrons. During recombination the free electron density drops sharply and the mean free path of the photons grows larger than the Hubble scale. At the temperature $T_{\mathrm{dec}} \sim 3000 \mathrm{~K}$ (corresponding to the redshift $z_{\mathrm{dec}} \simeq 1100$ and the physical time $t_{\mathrm{dec}}=a_{0} \eta_{\mathrm{dec}} \simeq 10^{5}$ years) photons become free and the universe becomes transparent.

After recombination, the photon distribution evolves according to Liouville's equation (geodesic spray):

$$
p^{\mu} \partial_{\mu} f-\Gamma_{\mu \nu}^{i} p^{\mu} p^{\nu} \frac{\partial f}{\partial p^{i}} \equiv L_{X_{g}} f=0
$$

where $i=1,2,3$. Since the photons are massless, $|\mathbf{p}|^{2}=$ $\sum_{i=1}^{3} p_{i} p^{i}=\omega^{2} \quad\left(\omega=a p^{0}\right)$. Isotropy of the distribution implies that $f$ depends on $p^{i}$ only via $|\mathbf{p}|=\omega$, and so

$$
\frac{\partial f}{\partial p^{i}}=\frac{\partial \omega}{\partial p^{i}} \frac{\partial f}{\partial \omega}=\frac{p^{i}}{\omega} \frac{\partial f}{\partial \omega} .
$$

In a Friedmann universe (also if $\kappa \neq 0$ !) we find for $p^{\mu} p_{\mu}=-\omega^{2}+\mathbf{p}^{2}=0$ [exercise!]

$$
p^{i} \partial_{i} f-\Gamma_{\mu \nu}^{i} p^{\mu} p^{\nu} p_{i} \frac{1}{\omega} \frac{\partial f}{\partial \omega}=-\omega^{2}\left(\frac{\dot{a}}{a^{2}}\right) \frac{\partial f}{\partial \omega} .
$$

Inserting this result into (1.21) leads to

$$
\partial_{\eta} f+\omega\left(\frac{\dot{a}}{a}\right) \frac{\partial f}{\partial \omega}=0
$$

which is satisfied by an arbitrary function $f=f(\omega a)$. Hence the distribution of free-streaming photons changes 
just by redshifting the momenta. Therefore, setting $T \propto$ $a^{-1}$ even after recombination, the blackbody shape of the photon distribution remains unchanged.

Note however that after recombination the photons are no longer in thermal equilibrium and the $T$ in the Planck distribution is not a temperature in the thermodynamical sense but merely a parameter in the photon distribution function.
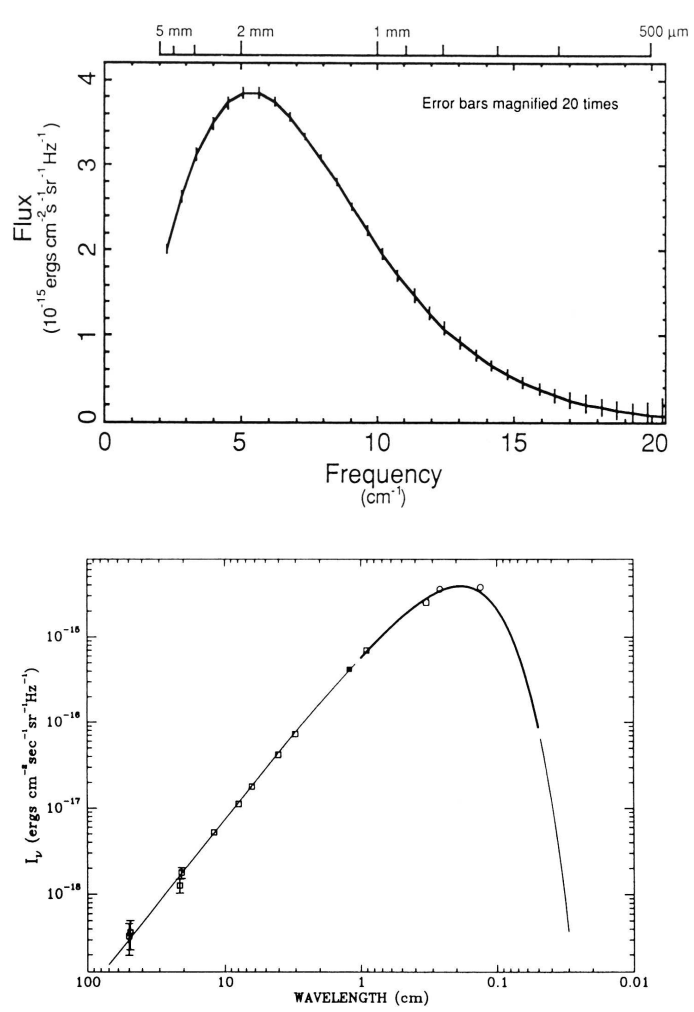

Fig. 4. Spectrum of the cosmic background radiation. The graph on the top shows the measurements of the FIRAS experiment on COBE (the vertical bars), overlaid by a blackbody spectrum at a temperature of $2.73 \mathrm{~K}$. The error bars are 20 times magnified! The image on the bottom shows a larger number of measurements. The FIRAS data is represented by the fat line around the peak of the spectrum (from Peebles [15]).

The blackbody spectrum of these cosmic photons which are called the "cosmic microwave background" (CMB) is extremely well verified observationally (see Fig. 4). The limits on deviations are often parameterized in terms of three parameters: The chemical potential $\mu$, the Compton $y$ parameter (which quantifies a well defined change in the spectrum arising from interactions with a non-relativistic electron gas at a different temperature, see, e.g. [15]) and $Y_{\mathrm{ff}}$ (describing a contamination by free-free emission).

The present limits on these parameters are (at $95 \%$ CL, [7])

$$
|\mu|<9 \cdot 10^{-5}, \quad|y|<1.2 \cdot 10^{-5}, \quad\left|Y_{\mathrm{ff}}\right|<1.9 \cdot 10^{-5} .
$$

The CMB photons have not only a very thermal spectrum, but they are also distributed very isotropically, apart from a dipole which is (most probably) simply due to our motion relative to the surface of last scattering:

An observer moving with velocity $\mathbf{v}$ relative to a source emitting a photon with proper momentum $\mathbf{p}=-\omega \mathbf{n}$ sees this photon redshifted with frequency

$$
\omega^{\prime}=\gamma \omega(1-\mathbf{n v})
$$

where $\gamma=\frac{1}{\sqrt{1-v^{2}}}$ is the relativistic $\gamma$-factor. For an isotropic emission of photons coming from all directions $\mathbf{n}$ this leads to a dipole anisotropy in first order in $\mathbf{v}$. This dipole anisotropy, which is of the order of

$$
\left(\frac{\Delta T}{T}\right)_{\text {dipole }} \simeq 10^{-3}
$$

has already been discovered in the 70ties [16,17]. Interpreting it as due to our motion with respect to the last scattering surface implies a velocity for the solar-system bary-center of $v=371 \pm 0.5 \mathrm{~km} / \mathrm{s}$ at $68 \% \mathrm{CL}([7])$.

The COBE [11] DMR experiment (Differential Microwave Radiometer) has found fluctuations of

$$
\sqrt{\left\langle\left(\frac{\Delta T}{T}\right)^{2}\right\rangle} \sim 10^{-5}
$$

on all angular scales $\theta \geq 7^{\circ}[8]$. On smaller angular scales many experiments have found fluctuations (we shall describe the experimental results in more detail later), but all of them are $\lesssim 10^{-4}$.

As we shall see later, the CMB fluctuations on large scales provide a measure for the deviation of the geometry from the Friedmann-Lemaitre one. The geometry perturbations are thus small and we may calculate their effects by linear perturbation theory. On smaller scales, $\Delta T / T$ reflects the fluctuations in the energy density in the baryon/radiation plasma prior to recombination. Their amplitude is just about right to allow the formation of the presently observed non-linear structures (like galaxies, clusters, etc.) out of small initial fluctuations by gravitational instability.

These findings strongly support the hypothesis which we assume here, namely that the large scale structure (i.e., galaxy distribution) observed in the universe formed by gravitational instability from relatively small $\left(\sim 10^{-4}-10^{-5}\right)$ initial fluctuations. As we shall see, such initial fluctuations leave an interesting "fingerprint" on the cosmic microwave background.

\section{PERTURBATION THEORY}

The tool for the analysis of CMB anisotropies is cosmological perturbation theory. We spend therefore some time on this subject, especially on the fundamental level. 


\section{R. DURRER}

Once all the variables are defined, we will be rather brief in the derivation of the basic perturbation equations. First of all, because these derivations are in general not very illuminating and secondly because nowadays all of you can obtain them very easily by setting

$$
g_{\mu \nu}=\bar{g}_{\mu \nu}+\varepsilon a^{2} h_{\mu \nu}
$$

$\left(\bar{g}_{\mu \nu}\right.$ being the unperturbed Friedmann metric) and asking Mathematica or Maple to calculate the Einstein Tensor using the condition $\varepsilon^{2}=0$. We conventionally set (absorbing the "smallness" parameter $\varepsilon$ into $h_{\mu \nu}$ )

$$
\begin{aligned}
& g_{\mu \nu}=\bar{g}_{\mu \nu}+a^{2} h_{\mu \nu}, \\
& \bar{g}_{00}=-a^{2}, \\
& \bar{g}_{i j}=a^{2} \gamma_{i j}, \\
& \left|h_{\mu \nu}\right| \ll 1 ; \\
& T_{\nu}^{\mu}=\bar{T}_{\nu}^{\mu}+\theta_{\nu}^{\mu}, \\
& \bar{T}_{0}^{0}=-\bar{\rho}, \\
& \bar{T}_{j}^{i}=\bar{p} \delta_{j}^{i}, \\
& \left|\theta_{\nu}^{\mu}\right| / \bar{\rho} \ll 1 .
\end{aligned}
$$

\section{A. Gauge transformation, gauge invariance}

The first fundamental problem we want to discuss is the problem of 'choice of gauge' in cosmological perturbation theory:

For linear perturbation theory to apply, the spacetime manifold $\mathcal{M}$ with metric $g$ and the energy momentum tensor $T$ of the real, observable universe must be in some sense close to a Friedmann universe, i.e., the manifold $\mathcal{M}$ with a Robertson-Walker metric $\bar{g}$ and a homogeneous and isotropic energy momentum tensor $\bar{T}$. It is an interesting, non-trivial unsolved problem how to construct $\bar{g}$ and $\bar{T}$ from the physical fields $g$ and $T$ in practice. There are two main difficulties: Spatial averaging procedures depend on the choice of a hyper-surface of constant time and do not commute with derivatives, so that averaged fields $\bar{g}$ and $\bar{T}$ will in general not satisfy Einstein's equations. Secondly, averaging is in practice impossible over super-horizon scales.

Even though we cannot give a constructive prescription, we now assume that there exists an averaging procedure which leads to a Friedmann universe with spatially averaged tensor fields $\bar{Q}$, such that the deviations $\left(T_{\mu \nu}-\right.$ $\left.\bar{T}_{\mu \nu}\right) / \max _{\{\alpha \beta\}}\left\{\left|\bar{T}_{\alpha \beta}\right|\right\}$ and $\left(g_{\mu \nu}-\bar{g}_{\mu \nu}\right) / \max _{\{\alpha \beta\}}\left\{\bar{g}_{\alpha \beta}\right\}$ are small, and $\bar{g}$ and $\bar{T}$ satisfy Friedmann's equations. Let us call such an averaging procedure 'admissible'. There may be many different admissible averaging procedures (e.g. over a different hyper-surface) leading to slightly different Friedmann backgrounds. But since $|g-\bar{g}|$ is small of order $\epsilon$, the difference of the two Friedmann backgrounds must also be small of order $\epsilon$ and we can regard it as part of the perturbation.
We consider now a fixed admissible Friedmann background $(\bar{g}, \bar{T})$ as chosen. Since the theory is invariant under diffeomorphisms (coordinate transformations), the perturbations are not unique. For an arbitrary diffeomorphism $\phi$ and its pullback $\phi^{*}$, the two metrics $g$ and $\phi^{*}(g)$ describe the same geometry. Since we have chosen the background metric $\bar{g}$ we only allow diffeomorphisms which leave $\bar{g}$ invariant, i.e., which deviate only in first order form the identity. Such an 'infinitesimal' isomorphism can be represented as the infinitesimal flow of a vector field $X, \phi=\phi_{\epsilon}^{X}$. Remember the definition of the flow: For the integral curve $\gamma_{x}(s)$ of $X$ with starting point $x$, i.e., $\gamma_{x}(s=0)=x$ we have $\phi_{s}^{X}(x)=\gamma_{x}(s)$. In terms of the vector field $X$, to first order in $\epsilon$, its pullback is then of the form

$$
\phi^{*}=i d+\epsilon L_{X}
$$

( $L_{X}$ denotes the Lie derivative in direction $\left.X\right)$. The transformation $g \rightarrow \phi^{*}(g)$ is equivalent to $\bar{g}+\epsilon a^{2} h \rightarrow$ $\bar{g}+\epsilon\left(a^{2} h+L_{X} \bar{g}\right)$, i.e. under an infinitesimal coordinate transformation' the metric perturbation $h$ transforms as

$$
h \rightarrow h+a^{-2} L_{X} \bar{g} .
$$

In the context of cosmological perturbation theory, infinitesimal coordinate transformations are called 'gauge transformation'. The perturbation of a arbitrary tensor field $Q=\bar{Q}+\epsilon Q^{(1)}$ obeys the gauge transformations law

$$
Q^{(1)} \rightarrow Q^{(1)}+L_{X} \bar{Q}
$$

Since every vector field $X$ generates a gauge transformation $\phi=\phi_{\epsilon}^{X}$, we can conclude that only perturbations of tensor fields with $L_{X} \bar{Q}=0$ for all vector fields $X$, i.e., with vanishing (or constant) 'background contribution' are gauge invariant. This simple result is sometimes referred to as the 'Stewart-Walker Lemma' [3].

The gauge dependence of perturbations has caused many controversies in the literature, since it is often difficult to extract the physical meaning of gauge dependent perturbations, especially on super-horizon scales. This has led to the development of gauge invariant perturbation theory which we are going to use throughout this review. The advantage of the gauge-invariant formalism is that the variables used have simple geometric and physical meanings and are not plagued by gauge modes. Although the derivation requires somewhat more work, the final system of perturbation equations is usually simple and well suited for numerical treatment. We shall also see, that on sub-horizon scales, the gauge invariant matter perturbations variables approach the usual, gauge dependent ones. Since one of the gauge invariant geometrical perturbation variables corresponds to the Newtonian potential, the Newtonian limit can be performed easily.

First we note that since all relativistic equations are covariant (i.e. can be written in the form $Q=0$ for some tensor field $Q$ ), it is always possible to express the corresponding perturbation equations in terms of gauge invariant variables $[4-6]$. 


\section{B. Gauge invariant perturbation variables}

Since the $\{\eta=$ const $\}$ hyper-surfaces are homogeneous and isotropic, it is sensible to perform a harmonic analysis: A (spatial) tensor field $Q$ on these hyper-surfaces can be decomposed into components which transform irreducibly under translations and rotations. All such components evolve independently. For a scalar quantity $f$ in the case $\kappa=0$ this is nothing else than its Fourier decomposition:

$$
f(\mathbf{x}, \eta)=\int d^{3} k \hat{f}(\mathbf{k}) e^{i \mathbf{k x}} .
$$

(The exponentials $Y_{\mathbf{k}}(\mathbf{x})=e^{i \mathbf{k x}}$ are the unitary irreducible representations of the Euclidean translation group.) For $\kappa=1$ such a decomposition also exists, but the values $k$ are discrete, $k^{2}=\ell(\ell+2)$ and for $\kappa=-1$, they are bounded from below, $k^{2}>1$. Of course, the functions $Y_{\mathbf{k}}$ are different for $\kappa \neq 0$.

They are always the complete orthogonal set of eigenfunctions of the Laplacian.

$$
\Delta Y^{(S)}=-k^{2} Y^{(S)} .
$$

In addition, a tensorial variable (at fixed position $\mathbf{x}$ ) can be decomposed into irreducible components under the rotation group $S O(3)$.

For a vector field, this is its decomposition into a gradient and a rotation,

$$
V_{i}=\nabla_{i} \varphi+B_{i}
$$

where

$$
B_{\mid i}^{i}=0,
$$

where we used $X_{\mid i}$ to denote the three-dimensional covariant derivative of $X . \varphi$ is the spin 0 and $\mathbf{B}$ is the spin 1 component of $V$.

For a symmetric tensor field we have

$$
\begin{aligned}
H_{i j} & =H_{L} \gamma_{i j}+\left(\nabla_{i} \nabla_{j}-\frac{1}{3} \Delta \gamma_{i j}\right) H_{T} \\
& +\frac{1}{2}\left(H_{i \mid j}^{(V)}+H_{j \mid i}^{(V)}\right)+H_{i j}^{(T)},
\end{aligned}
$$

where

$$
H_{i}^{(V) \mid i}=H_{i}^{(T)^{i}}=H_{i \mid j}^{(T)^{j}}=0 .
$$

Here $H_{L}$ and $H_{T}$ are spin 0 components, $H_{i}^{(V)}$ is a spin 1 component and $H_{i j}^{(T)}$ is a spin 2 component.

We shall not need higher tensors (or spinors). As a basis for vector and tensor modes we use the vector and tensor type eigenfunctions to the Laplacian,

$$
\Delta Y_{j}^{(V)}=-k^{2} Y_{j}^{(V)},
$$

and

$$
\Delta Y_{j i}^{(T)}=-k^{2} Y_{j i}^{(T)}
$$

where $Y_{j}^{(V)}$ is a transverse vector, $Y_{j}^{(V) \mid j}=0$ and $Y_{j i}^{(T)}$ is a symmetric transverse traceless tensor, $Y_{j}^{(T) j}=$ $Y_{j i}^{(T) \mid i}=0$.

According to Eqs. (2.7) and (2.9) we can construct scalar type vectors and tensors and vector type tensors. To this goal we define

$$
\begin{aligned}
Y_{j}^{(S)} & \equiv-k^{-1} Y_{\mid j}^{(S)} \\
Y_{i j}^{(S)} & \equiv k^{-2} Y_{\mid i j}^{(S)}+\frac{1}{3} \gamma_{i j} Y^{(S)}, \\
Y_{i j}^{(V)} & \equiv-\frac{1}{2 k}\left(Y_{i \mid j}^{(V)}+Y_{j \mid i}^{(V)}\right) .
\end{aligned}
$$

In the following we shall extensively use this decomposition and write down the perturbation equations for a given mode $k$.

The decomposition of a vector field is then of the form

$$
B_{i}=B Y_{i}^{(S)}+B^{(V)} Y_{i}^{(V)} .
$$

The decomposition of a tensor field is given by (compare (2.9))

$$
H_{i j}=H_{L} Y^{(S)} \gamma_{i j}+H_{T} Y_{i j}^{(S)}+H^{(V)} Y_{i j}^{(V)}+H^{(T)} Y_{i j}^{(T)},
$$

where $B, B_{i}^{(V)}, H_{L}, H_{T}, H_{i}^{(V)}$ and $H_{i j}^{(T)}$ are functions of $\eta$ and $\mathbf{k}$

\section{Metric perturbations}

Perturbations of the metric are of the form

$$
g_{\mu \nu}=\bar{g}_{\mu \nu}+a^{2} h_{\mu \nu} .
$$

We parameterize them as

$$
h_{\mu \nu} d x^{\mu} d x^{\nu}=-2 A d \eta^{2}-2 B_{i} d \eta d x^{i}+2 H_{i j} d x^{i} d x^{j},
$$

and we decompose the perturbation variables $B_{i}$ and $H_{i j}$ according to (2.16) and (2.17).

Let us consider the behavior of $h_{\mu \nu}$ under gauge transformations. We set the vector field defining the gauge transformation to

$$
\mathbf{X}=T \partial_{\eta}+L^{i} \partial_{i}
$$

Using simple identities from differential geometry like

$$
L_{\mathbf{X}}(d f)=d\left(L_{\mathbf{X}} f\right)
$$

and

$$
\left(L_{\mathrm{X}} \gamma\right)_{i j}=X_{i \mid j}+X_{j \mid i}
$$

we obtain 


\section{R. DURRER}

$$
\begin{aligned}
L_{\mathbf{X}} \bar{g}= & a^{2}\left[-2\left(\frac{\dot{a}}{a} T+\dot{T}\right) d \eta^{2}+2\left(\dot{L}_{i}-T_{, i}\right) d \eta d x^{i}\right. \\
& \left.+\left(2 \frac{\dot{a}}{a} T \gamma_{i j}+L_{i \mid j}+L_{j \mid i}\right) d x^{i} d x^{j}\right]
\end{aligned}
$$

Comparing this with (2.19) and using (2.3) we obtain the following behavior of our perturbation variables under gauge transformations (decomposing $L_{i}=$ $\left.L Y_{i}^{(S)}+L^{(V)} Y_{i}^{(V)}\right)$ :

$$
\begin{aligned}
& A \rightarrow A+\frac{\dot{a}}{a} T+\dot{T}, \\
& B \rightarrow B-\dot{L}-k T, \\
& B^{(V)} \rightarrow B^{(V)}-\dot{L}^{(V)}, \\
& H_{L} \rightarrow H_{L}+\frac{\dot{a}}{a} T+\frac{k}{3} L, \\
& H_{T} \rightarrow H_{T}-k L, \\
& H^{(V)} \rightarrow H^{(V)}-k L^{(V)}, \\
& H^{(T)} \rightarrow H^{(T)} .
\end{aligned}
$$

Two scalar and one vector variable can be brought to disappear by gauge transformations.

One often chooses $k L=H_{T}$ and $T=B+\dot{L}$, so that the variables $H_{T}$ and $B$ vanish. In this gauge (longitudinal gauge), scalar perturbations of the metric are of the form $\left(H_{T}=B=0\right)$ :

$$
h_{\mu \nu}^{(S)}=-2 \Psi d \eta^{2}+2 \Phi \gamma_{i j} d x^{i} d x^{j} .
$$

$\Psi$ and $\Phi$ are the so called Bardeen potentials. In general they are defined by

$$
\begin{aligned}
& \Psi=A-\frac{\dot{a}}{a} k^{-1} \sigma-k^{-1} \dot{\sigma}, \\
& \Phi=H_{L}+\frac{1}{3} H_{T}-\frac{\dot{a}}{a} k^{-1} \sigma
\end{aligned}
$$

with $\sigma=k^{-1} \dot{H}_{T}-B$. A short calculation using Eqs. $(2.22)$ to $(2.26)$ shows that they are gauge invariant.

For vector perturbations it is convenient to set $k L^{(V)}=H^{(V)}$ so that $H^{(V)}$ vanishes and we have

$$
h_{\mu \nu}^{(V)} d x^{\mu} d x^{\nu}=2 \sigma^{(V)} Y_{i}^{(V)} d \eta d x^{i} .
$$

We shall call this gauge the "vector gauge". In general $\sigma^{(V)}=k^{-1} \dot{H}^{(V)}-B^{(V)}$ is gauge invariant [12]

Clearly there are no tensorial (spin 2) gauge transformation and hence $H_{i j}^{(T)}$ is gauge invariant.

\section{Perturbations of the energy momentum tensor}

Let $T_{\nu}^{\mu}=\bar{T}_{\nu}^{\mu}+\Theta_{\nu}^{\mu}$ be the full energy momentum tensor. We define its energy density $\rho$ and its energy flow 4 -vector $u$ as the time-like eigenvalue and eigenvector of $T_{\nu}^{\mu}$ :

$$
T_{\nu}^{\mu} u^{\nu}=-\rho u^{\mu}, \quad u^{2}=-1 .
$$

We then define their perturbations by

$$
\rho=\bar{\rho}(1+\delta), \quad u=u^{0} \partial_{t}+u^{i} \partial_{i} .
$$

$u^{0}$ is fixed by the normalization condition,

$$
u^{0}=\frac{1}{a}(1-A) .
$$

We further set

$$
u^{i}=\frac{1}{a} v^{i}=v Y^{(S) i}+v^{(V)} Y^{(V) i} .
$$

We define $P_{\nu}^{\mu} \equiv u^{\mu} u_{\nu}+\delta_{\nu}^{\mu}$, the projection tensor onto the part of tangent space normal to $u$ and set the stress tensor

$$
\tau^{\mu \nu}=P_{\alpha}^{\mu} P_{\beta}^{\nu} T^{\alpha \beta}
$$

In the unperturbed case we have $\tau_{0}^{0}=0, \tau_{j}^{i}=\bar{p} \delta_{j}^{i}$. Including perturbations, to first order we still obtain

$$
\tau_{0}^{0}=\tau_{i}^{0}=\tau_{0}^{i}=0 .
$$

But $\tau_{j}^{i}$ contains in general perturbations. We set

$$
\tau_{j}^{i}=\bar{p}\left[\left(1+\Pi_{L}\right) \delta_{j}^{i}+\Pi_{j}^{i}\right], \quad \text { with } \quad \Pi_{i}^{i}=0 .
$$

We decompose $\Pi_{j}^{i}$ as

$$
\Pi_{j}^{i}=\Pi^{(S)} Y_{j}^{(S) i}+\Pi^{(V)} Y_{j}^{(V) i}+\Pi^{(T)} Y_{j}^{(T) i} .
$$

We shall not derive the gauge transformation properties in detail, but just state some results which can be obtained as an exercise (see also [5]):

- Of the variables defined above only the $\Pi^{(S, V, T)}$ are gauge invariant; they describe the anisotropic stress tensor, $\Pi_{\nu}^{\mu}=\tau_{\nu}^{\mu}-1 / 3 \tau_{\alpha}^{\alpha} \delta_{\nu}^{\mu}$. They are gauge invariant due to the Stewart-Walker lemma, since $\bar{\Pi}=0$. For perfect fluids $\Pi_{\nu}^{\mu}=0$.

- A second gauge invariant variable is

$$
\Gamma=\pi_{L}-\frac{c_{s}^{2}}{w} \delta
$$

where $c_{s}^{2} \equiv \dot{p} / \dot{\rho}$ is the adiabatic sound speed and $w \equiv p / \rho$ is the enthalpy. One can show that $\Gamma$ is proportional to the divergence of the entropy flux of the perturbations. Adiabatic perturbations are characterized by $\Gamma=0$.

- Gauge invariant density and velocity perturbations can be found by combining $\delta, v$ and $v_{i}^{(V)}$ with metric perturbations. 
We shall use

$$
\begin{aligned}
V & \equiv v-\frac{1}{k} \dot{H}_{T}=v^{(\text {long })} \\
D_{g} & \equiv \delta+3(1+w)\left(H_{L}+\frac{1}{3} H_{T}\right)=\delta^{(\text {long })} \\
& +3(1+w) \Phi \\
D & \equiv \delta^{(\text {long })}+3(1+w)\left(\frac{\dot{a}}{a}\right) \frac{V}{k} \\
V^{(V)} & \equiv v^{(V)}-\frac{1}{k} \dot{H}^{(V)}=v^{(\text {vec })} \\
\Omega & \equiv v^{(V)}-B^{(V)}=v^{(\text {vec })}-B^{(V)} \\
\Omega & -V^{(V)}=\sigma^{(V)}
\end{aligned}
$$

Here $v^{\text {(long) }}, \delta^{\text {(long) }}$ and $v_{i}^{\text {(vec) }}$ are the velocity (and density) perturbations in the longitudinal and vector gauge respectively and $\sigma^{(V)}$ is the metric perturbation in vector gauge (see Eq. (2.32)). These variables can be interpreted nicely in terms of gradients of the energy density and the shear and vorticity of the velocity field [18].

But we just want to show that on scales much smaller than the Hubble scale, $k \eta \gg 1$, the metric perturbations are much smaller than $\delta$ and $v$ and we can thus "forget them" (which will be important when comparing experimental results with calculations in this formalism):

The perturbations of the Einstein tensor are given by second derivatives of the metric perturbations. Einstein's equations yield the following order of magnitude estimate:

$$
\begin{aligned}
& \mathcal{O}\left(\frac{\delta T}{T}\right) \underbrace{\mathcal{O}(8 \pi G T)}_{\mathcal{O}\left(\frac{\dot{a}}{a}\right)^{2}=\mathcal{O}\left(\eta^{-2}\right)}=\mathcal{O}\left(\frac{1}{\eta^{2}} h+\frac{k}{\eta} h+k^{2} h\right), \\
& \mathcal{O}\left(\frac{\delta T}{T}\right)=\mathcal{O}\left(h+k \eta h+(k \eta)^{2} h\right)
\end{aligned}
$$

For $k \eta \gg 1$ this gives $\mathcal{O}(\delta, v)=\mathcal{O}\left(\frac{\delta T}{T}\right) \gg \mathcal{O}(h)$. On sub-horizon scales the difference between $\delta, \delta^{\text {(long) }}, D_{g}$ and $D$ is negligible as well as the difference between $v$ and $V$ or $v^{(V)}, V^{(V)}$ and $\Omega^{(V)}$.

Later we shall also need other perturbation variables like the perturbation of the photon brightness (energyintegrated photon distribution function), but we shall introduce them as we get there and discuss some applications first.

\section{Basic perturbation equations}

As already announced, we do not derive Einstein's equations but just write down those which we shall need later:

\section{Constraint equations}

$$
\begin{aligned}
& 4 \pi G a^{2} \rho D=\left(k^{2}-3 \kappa\right) \Phi \\
& 4 \pi G a^{2}(\rho+p) V=k\left(\left(\frac{\dot{a}}{a}\right) \Psi-\dot{\Phi}\right) \\
& 8 \pi G a^{2}(\rho+p) \Omega=\frac{1}{2}\left(2 \kappa-k^{2}\right) \sigma^{(V)}
\end{aligned}
$$

\section{Dynamical equations}

$$
\begin{array}{ll}
-k^{2}(\Phi+\Psi)=8 \pi G a^{2} p \Pi^{(S)}, & \text { (scalar) } \\
k\left(\dot{\sigma}^{(V)}+2\left(\frac{\dot{a}}{a}\right) \sigma^{(V)}\right)=8 \pi G a^{2} p \Pi^{(V)}, & \text { (vector) } \\
\ddot{H}^{(T)}+2\left(\frac{\dot{a}}{a}\right) \dot{H}^{(T)}+\left(2 \kappa+k^{2}\right) H^{(T)}=8 \pi G a^{2} p \Pi_{i j}^{(T)} . & \text { (tensor) }
\end{array}
$$

There is a second dynamical scalar equations, which is however complicated and not needed, since we may instead use one of the conservation equations below. Note that for perfect fluids, where $\Pi_{j}^{i} \equiv 0$, we have $\Phi=-\Psi, \sigma^{(V)} \propto 1 / a^{2}$ and $H$ obeys a damped wave equation. The damping term can be neglected on small scales (over short time periods) when $\eta^{-2} \lesssim 2 \kappa+k^{2}$, and $H_{i j}$ represents propagating gravitational waves. For vanishing curvature, these are just the sub-horizon scales, $k \eta \gtrsim 1$. For $\kappa<0$, waves oscillate with a somewhat smaller frequency, $\omega=\sqrt{2 \kappa+k^{2}}$, while for $\kappa>0$ the frequency is somewhat larger. 


\section{R. DURRER}

\section{Conservation equations}

The conservation equations, $T_{; \nu}^{\mu \nu}=0$ lead to the following perturbation equations

$$
\begin{aligned}
& \dot{D}_{g}+3\left(c_{s}^{2}-w\right)\left(\frac{\dot{a}}{a}\right) D_{g}+(1+w) k V+3 w\left(\frac{\dot{a}}{a}\right) \Gamma=0, \\
& \dot{V}+\left(\frac{\dot{a}}{a}\right)\left(1-3 c_{s}^{2}\right) V=k\left(\Psi-3 c_{s}^{2} \Phi\right)+\frac{c_{s}^{2}}{1+w} D_{g}+\frac{w k}{1+w}\left[\Gamma-\frac{2}{3}\left(1-\frac{3 \kappa}{k^{2}}\right) \Pi\right], \\
& \dot{\Omega}_{i}+\left(1-3 c_{s}^{2}\right)\left(\frac{\dot{a}}{a}\right) \Omega_{i}=\frac{p}{2(\rho+p)}\left(k-\frac{2 \kappa}{k}\right) \Pi_{i}^{(V)}
\end{aligned}
$$

\section{SIMPLE APPLICATIONS}

We first discuss some simple applications which will be important for the CMB. We could of course also write $(2.55)$ in terms of $D$, but we shall just work with the relation

$$
D=D_{g}+3(1+w)\left(-\Phi+\left(\frac{\dot{a}}{a}\right) k^{-1} V\right) .
$$

\section{A. The pure dust fluid at $\kappa=0, \Lambda=0$}

We assume the dust to have $w=c_{s}^{2}=p=0$ and $\Pi=\Gamma=0$. The equations (2.55), (2.52) and (2.50) then reduce to

$$
\begin{array}{ll}
\dot{D}_{g}=-k V, & \text { (energy conservation eqn.) } \\
\dot{V}+\left(\frac{\dot{a}}{a}\right) V=k \Psi, & \text { (gravitational acceleration eqn.) } \\
\Phi=-\Psi & \\
-k^{2} \Psi=4 \pi G a^{2} \rho\left(D_{g}+3\left(\Psi+\left(\frac{\dot{a}}{a}\right) k^{-1} V\right)\right) . & \text { (Poisson eqn.) }
\end{array}
$$

In a pure dust universe $\rho \propto a^{-3} \Rightarrow(\dot{a} / a)^{2} \propto a^{-1}$, which is solved by $a \propto \eta^{2}$. The Einstein equations then give immediately $4 \pi G \rho a^{2}=3 / 2(\dot{a} / a)^{2}=6 / \eta^{2}$. Setting $k \eta=x$ and ${ }^{\prime}=d / d x$, the system $(3.2-3.5)$ then becomes

$$
\begin{aligned}
& D_{g}^{\prime}=-V, \\
& V^{\prime}+\frac{2}{x} V=\Psi, \\
& \frac{6}{x^{2}}\left(D_{g}+3\left(\Psi+\frac{2}{x} V\right)\right)=-\Psi .
\end{aligned}
$$

We use (3.8) to eliminate $\Psi$ and (3.6) to eliminate $D_{g}$, leading to

$$
\left(18+x^{2}\right) V^{\prime \prime}+\left(\frac{72}{x}+4 x\right) V^{\prime}-\left(\frac{72}{x^{2}}+4\right) V=0 .
$$

The general solution of Eq. (3.9) is

$$
V=V_{0} x+\frac{V_{1}}{x^{4}}
$$

with arbitrary constants $V_{0}$ and $V_{1}$. Since the perturbations are supposed to be small initially, they cannot diverge for $x \rightarrow 0$, and we have therefore to choose $V_{1}=0$ (the growing mode). Another way to argue is as follows: If the mode $V_{1}$ has to be small already at some early initial time $\eta_{\text {in }}$, it will be even much smaller at later times and may hence be neglected. The perturbation variables are then given by

$$
\begin{aligned}
& V=V_{0} x, \\
& D_{g}=-15 V_{0}-\frac{1}{2} V_{0} x^{2}, \\
& \Psi=3 V_{0} .
\end{aligned}
$$

The constancy of the gravitational potential $\Psi$ in a matter dominated universe and the growth of the density perturbations like the scale factor $a$ led Lifshitz to conclude 1946 [19] that pure gravitational instability cannot be the cause for structure formation: If we start from tiny thermal fluctuations of the order of $10^{-35}$, they can only grow to about $10^{-30}$ through this process during 
the matter dominated regime. Or, to put it differently, if we do not want to modify the process of structure formation, we need initial fluctuations of the order of at least $10^{-5}$. One possibility to create such fluctuations is due to quantum particle production in the classical gravitational field during inflation. The rapid expansion of the universe during inflation quickly transforms microscopic scales at which quantum fluctuations are important into cosmological scales where these fluctuations are then "frozen in" as classical perturbations in the energy density and the geometry.

We distinguish two regimes:

i) super-horizon, $x \ll 1$ where we have

$$
\begin{aligned}
& D_{g}=-15 V_{0}, \\
& \Psi=3 V_{0}, \\
& V=V_{0} x
\end{aligned}
$$

and $i$ ) sub-horizon, $x \gg 1$ where the solution is dominated by the terms

$$
\begin{aligned}
& V=V_{0} x, \\
& D_{g}=-\frac{1}{2} V_{0} x^{2}, \\
& \Psi=3 V_{0}=\text { const. }
\end{aligned}
$$

Note that for dust

$$
D=D_{g}+3 \Psi+\frac{6}{x} V=-\frac{1}{2} V_{0} x^{2} .
$$

In the variable $D$ the constant term has disappeared and we have $D \ll \Psi$ on super horizon scales, $x \ll 1$.

\section{B. The pure radiation fluid, $\kappa=0, \Lambda=0$}

In this limit we set $w=c_{s}^{2}=1 / 3$ and $\Pi=0$. We conclude from $\rho \propto a^{-4}$ that $a \propto \eta$ and $\Phi=-\Psi$, and the perturbation equations become (with the same notation as above):

$$
\begin{aligned}
& D_{g}^{\prime}=-\frac{4}{3} V, \\
& V^{\prime}=2 \Psi+\frac{1}{4} D_{g}, \\
& -2 x^{2} \Psi=3 D_{g}+12 \Psi+\frac{12}{x} V .
\end{aligned}
$$

The general solution of this system is

$$
\begin{aligned}
D_{g}= & D_{2}\left[\cos \left(\frac{x}{\sqrt{3}}\right)-2 \frac{\sqrt{3}}{x} \sin \left(\frac{x}{\sqrt{3}}\right)\right] \\
& +D_{1}\left[\sin \left(\frac{x}{\sqrt{3}}\right)+2 \frac{\sqrt{3}}{x} \cos \left(\frac{x}{\sqrt{3}}\right)\right], \\
V= & -\frac{3}{4} D_{g}^{\prime}, \\
\Psi= & \frac{-3 D_{g}-(12 / x) V}{12+2 x^{2}} .
\end{aligned}
$$

Again, regularity at $x=0$ requires $D_{1}=0$.

In the super-horizon, $x \ll 1$ regime we obtain

$$
\Psi=\Psi_{0}, \quad D_{g}=D_{0}-\frac{2}{3} V_{0} x^{2}, \quad V=V_{0} x
$$

with

$$
\begin{aligned}
& D_{0}=-6 \Psi_{0}=-D_{2}, \\
& V_{0}=\frac{1}{2} \Psi_{0}=-\frac{1}{12} D_{0} .
\end{aligned}
$$

On sub-horizon, $x \gg 1$ scales we find oscillating solutions with constant amplitude with a frequency of $1 / \sqrt{3}$ :

$$
\begin{aligned}
& V=V_{2} \sin \left(\frac{x}{\sqrt{3}}\right), \\
& D_{g}=D_{2} \cos \left(\frac{x}{\sqrt{3}}\right), \quad \Psi=-\frac{3}{2} x^{-2} D_{g}, \\
& D_{2}=\frac{4 V_{2}}{\sqrt{3}}
\end{aligned}
$$

Note that also for radiation perturbations

$$
D=-\frac{2}{3} V_{0} x^{2} \ll \Psi
$$

is small on super horizon scales, $x \ll 1$. The perturbation amplitude is given by the largest gauge invariant perturbation variable. We conclude therefore that perturbations outside the Hubble horizon are frozen to first order. Once they enter the horizon they start to collapse, but pressure resists the gravitational force and the radiation fluid starts to oscillate. The perturbations of the gravitational potential oscillate and decay like $1 / a^{2}$ inside the horizon.

\section{Adiabatic and isocurvature initial conditions for} a matter \& radiation fluid

In this section we want to investigate a system with a matter and a radiation component that are coupled only by gravity. The matter component acts therefore as dark matter, since it does not interact directly with the radiation.

Since the matter and radiation perturbations behave in the same way on super-horizon scales,

$$
D_{g}^{(r)}=A+B x^{2}, D_{g}^{(m)}=A^{\prime}+B^{\prime} x^{2}, V^{(r)} \propto V^{(m)} \propto x,
$$

we may require a constant relation between matter and radiation perturbations. As we have seen in the previous section, inside the horizon $(x>1)$ radiation perturbations start to oscillate while matter perturbations keep following a power law. On sub-horizon scales a constant ratio can thus no longer be maintained. There are two interesting possibilities: 


\section{R. DURRER}

\section{Adiabatic initial conditions}

Adiabaticity requires that matter and radiation perturbations are initially in perfect thermal equilibrium. This implies that their velocity fields agree (see below, section on Boltzmann equation!)

$$
V^{(r)}=V^{(m)},
$$

so that the energy flux in the two fluids is coupled initially.

Let us investigate the radiation solution in the matter dominated era, when the corresponding scale is already sub-horizon. Since $\Psi$ is dominated by the matter contribution, we have $\Psi \simeq$ const $=\Psi_{0}$. We neglect the (decaying) contribution from the sub-dominant radiation to $\Psi$. Energy-momentum conservation for radiation then gives

$$
\begin{aligned}
D_{g}^{(r) \prime} & =-\frac{4}{3} V^{(r)} \\
V^{(r) \prime} & =2 \Psi+\frac{1}{4} D_{g}^{(r)} .
\end{aligned}
$$

Now $\Psi$ is just a constant given by the matter perturbations, and it acts like a constant source term. The full solution of this system is then

$$
\begin{aligned}
D_{g}^{(r)} & =A \cos \left(\frac{x}{\sqrt{3}}\right)-\frac{4}{\sqrt{3}} B \sin \left(\frac{x}{\sqrt{3}}\right) \\
& -8 \Psi\left[\cos \left(\frac{x}{\sqrt{3}}\right)-1\right], \\
V^{(r)} & =B \cos \left(\frac{x}{\sqrt{3}}\right)+\frac{\sqrt{3}}{4} A \sin \left(\frac{x}{\sqrt{3}}\right) \\
& -2 \sqrt{3} \Psi \sin \left(\frac{x}{\sqrt{3}}\right) .
\end{aligned}
$$

Our adiabatic initial conditions require

$$
\lim _{x \rightarrow 0} \frac{V^{(r)}}{x}=V_{0}=\lim _{x \rightarrow 0} \frac{V^{(m)}}{x}<\infty .
$$

Therefore $B=0$ and $A=4 V_{0}-8 \Psi$. Using in addition $\Psi=3 V_{0}($ see $(3.19))$ we obtain

$$
\begin{aligned}
& D_{g}^{(r)}=-\frac{44}{3} \Psi \cos \left(\frac{x}{\sqrt{3}}\right)+8 \Psi, \\
& V^{(r)}=\frac{1}{\sqrt{3}} \Psi \sin \left(\frac{x}{\sqrt{3}}\right), \\
& D_{g}^{(m)}=-\Psi\left(5+\frac{1}{6} x^{2}\right), \\
& V^{(m)}=\frac{1}{3} \Psi x, \\
& \Psi=3 V_{0} .
\end{aligned}
$$

On super-horizon scales, $x \ll 1$ we have

$$
D_{g}^{(r)} \simeq-\frac{20}{3} \Psi, \quad V^{(r)} \simeq \frac{1}{3} x \Psi,
$$

note that $D_{g}^{(r)}=(4 / 3) D_{g}^{(m)}$ and $V^{(r)}=V^{(m)}$ for adiabatic initial conditions.

\section{Isocurvature initial conditions}

Here we want to solve the system (2.50) and (2.55) for dark matter and radiation under the condition that the metric perturbations vanish initially, i.e., $\Psi=0$,

$$
\begin{aligned}
\Psi & =-\frac{3}{2}\left(\frac{\dot{a}}{a}\right)^{2} k^{-2}\left[D_{g}+3(1+w) \Psi\right. \\
& \left.+3(1+w)\left(\frac{\dot{a}}{a}\right) k^{-1} V\right]=0 .
\end{aligned}
$$

In principle, we have four evolution and one constraint equations. We therefore have four constants to adjust. Condition (3.45), however, requires an entire function to vanish. This may be impossible. Let us nevertheless try:

If $\Psi=0$ the solutions of the radiation dominated equations are simply

$$
\begin{aligned}
& D_{g}^{(r)}=A \cos \left(\frac{x}{\sqrt{3}}\right)+B \sin \left(\frac{x}{\sqrt{3}}\right), \\
& V^{(r)}=\frac{\sqrt{3}}{4} A \sin \left(\frac{x}{\sqrt{3}}\right)-\frac{\sqrt{3}}{4} B \cos \left(\frac{x}{\sqrt{3}}\right) .
\end{aligned}
$$

For the matter perturbations we find

$$
\begin{array}{ll}
V^{(m)}=-\frac{V_{0}}{a}, \quad a \propto x^{\beta}, & 1 \leq \beta \leq 2, \\
D_{g}^{(m)}=C^{(m)}-\frac{V_{0}}{\beta-1} \frac{x}{a} \quad \text { if } \beta \neq 1, \\
D_{g}^{(m)}=C^{(m)}-V_{0} \log (x) \quad \text { if } \beta=1 .
\end{array}
$$

Here $\beta$ is the exponent of the scale factor $a \propto \eta^{\beta}$, hence $\beta=1$ in the radiation era and $\beta=2$ in the matter era. $\Psi=0$ implies with

$$
\begin{aligned}
& D_{g}=\frac{1}{\rho}\left(\rho_{r} D_{g}^{(r)}+\rho_{m} D_{g}^{(m)}\right) \\
& V=\frac{1}{\rho+p}\left(\left(\rho_{r}+p_{r}\right) V^{(r)}+\rho_{m} V^{(m)}\right)
\end{aligned}
$$

that

$$
\begin{aligned}
0 & =\frac{\rho_{r}}{\rho_{m}} D_{g}^{(r)}+D_{g}^{(m)} \\
& +\left(\frac{\dot{a}}{a}\right) k^{-1}\left[\frac{4 \rho_{r}}{\rho_{m}} V^{(r)}+3 V^{(m)}\right] .
\end{aligned}
$$

Since $V^{(m)} \propto 1 / a$ it can compensate, for small values of $x$, the term $\propto \cos (x / \sqrt{3})$ of $V^{(r)}$, which behaves like $1 / a$ as well, due to the pre-factor $\rho_{r} / \rho_{m}$. This term can also be compensated in $D_{g}^{(r)}$ by the term $V_{0} x / a$ of $D_{g}^{(m)}$. In the purely radiation dominated universe, the log-dependence of $D_{g}^{(m)}$ renders this compensation imperfect. However, there is no way to compensate $C^{(m)}$ or the term proportional to $A$. We therefore have to choose $A=C^{(m)}=0$ and 


$$
a \frac{\rho_{r}}{\rho_{m}} \frac{\sqrt{3}}{3} B=V_{0}, \quad B=\frac{\rho_{m}}{a \rho_{r}} \sqrt{3} V_{0}
$$

(The compensation of the smaller terms in $D_{g}^{(r)}$ and $D_{g}^{(m)}$ is only complete if $\beta \simeq 2$.)

With $c_{s}=1 / \sqrt{3}$ we find

$$
\begin{aligned}
& D_{g}^{(r)} \simeq \frac{\rho_{m}}{a \rho_{r} c_{s}} V_{0} \sin \left(c_{s} x\right), \quad \text { (isocurvature) } \\
& D_{g}^{(r)} \simeq \Psi\left(8-\frac{44}{3} \cos \left(c_{s} x\right)\right) . \quad \text { (adiabatic) }
\end{aligned}
$$

The CMB anisotropies, which we are going to determine in the next section, contain a term

$$
\frac{\Delta T}{T}\left(\mathbf{k}, \eta_{0}, \mathbf{n}\right)=\cdots+\frac{1}{4} D_{g}^{(r)}\left(\mathbf{k}, \eta_{\text {dec }}\right) e^{i \mathbf{k n}\left(\eta_{0}-\eta_{\text {dec }}\right)} \cdots
$$

On scales where this term dominates, the peaks in $D_{g}$ translate into peaks in the angular power spectrum of CMB anisotropies.

For isocurvature initial conditions, we find a first peak in $D_{g}$ at

$$
\begin{aligned}
x_{i}^{(1)} & =k_{i}^{(1)} \eta_{\mathrm{dec}}=\frac{1}{c_{s}} \frac{\pi}{2}, \\
\lambda_{i}^{(1)} & =\frac{\pi}{k_{i}^{(1)}}=2 c_{s} \eta_{\mathrm{dec}} \\
\vartheta_{i}^{(1)} & \simeq \frac{2 c_{s} \eta_{\mathrm{dec}}}{\chi\left(\eta_{0}-\eta_{\mathrm{dec}}\right)} .
\end{aligned}
$$

Here $\vartheta_{i}^{(1)}$ is the angle under which the comoving scale $\lambda_{i}^{(1)}$ at comoving distance $\eta_{0}-\eta_{\text {dec }}$ is seen. In the next section, we will expand the temperature fluctuations in terms of spherical harmonics. An fluctuation on the angular scale $\vartheta$ then shows up around the harmonic $\ell \sim \pi / \vartheta$. As an indication, we note that for $\Lambda=\kappa=0$, the harmonic of the first isocurvature peak is

$$
\ell_{i}^{(1)} \sim \pi / \vartheta_{i}^{(1)} \sim 110
$$

In the adiabatic case the first "peak" is at $k_{a}^{(1)}=0$.

Since $D_{g}^{(r)}$ is negative for small $x$, the first peaks are "expansion peaks", and due to the gravitational attraction of the baryons (which we have neglected in this simple argument) they are less pronounced than the second ("compression") peaks.

These second peaks are usually called the "first acoustic peak". (It is the first compression peak and we shall adopt the convention to call it the "first peak" mainly for consistency with the literature.) They correspond to wavelengths and angular scales

$$
\begin{aligned}
& \lambda_{i}^{(2)}= \frac{2}{3} c_{s} \eta_{\mathrm{dec}}, \quad \vartheta_{i}^{(2)} \simeq \frac{(2 / 3) c_{s} \eta_{\mathrm{dec}}}{\chi\left(\eta_{0}-\eta_{\mathrm{dec}}\right)}, \\
& \ell_{i}^{(2)} \sim 350 \quad(\text { isocurvature }) \\
& \lambda_{a}^{(2)}=c_{s} \eta_{\mathrm{dec}}, \quad \vartheta_{a}^{(2)} \simeq \frac{c_{s} \eta_{\mathrm{dec}}}{\chi\left(\eta_{0}-\eta_{\mathrm{dec}}\right)}, \\
& \ell_{a}^{(2)} \sim 200 \quad \text { (adiabatic) } .
\end{aligned}
$$

Here the indicated harmonic is the one obtained in the case $\Lambda=\kappa=0$, for a typical baryon density inferred from nucleosynthesis.

It is interesting to note that the distance between consecutive peaks is independent of the initial condition. It is given by

$$
\begin{aligned}
& \Delta k_{i}=k_{i}^{(2)}-k_{i}^{(1)}=\pi /\left(c_{s} \eta_{\mathrm{dec}}\right)=\Delta k_{a}, \\
& \Delta \vartheta=\frac{c_{s} \eta_{\mathrm{dec}}}{\chi\left(\eta_{0}-\eta_{\mathrm{dec}}\right)}, \quad \Delta \ell \sim 200 .
\end{aligned}
$$

Again, the numerical value indicated for $\Delta \ell$ corresponds to a universe with $\Lambda=\kappa=0$. The result is strongly dependent especially on $\kappa$. This is the reason why the measurement of the peak position (or better of the inter-peak distance) allows an accurate determination of curvature.

From our analysis we can draw the following important conclusions: For scales where the $D_{g}^{(r)}$-term dominates, the CMB anisotropies show a series of acoustic oscillations with spacing $\Delta k$, the position of the first significant peaks is at $k=k_{a / i}^{(2)}$, depending on the initial condition.

The spacing $\Delta k$ is independent of initial conditions. The angle $\Delta \vartheta$ onto which this scale is projected in the sky is determined entirely by the matter content and the geometry of the universe. According to our findings in Section I, $\vartheta$ will be larger if $\Omega_{\kappa}<0$ (positive curvature) and smaller if $\Omega_{\kappa}>0$ (see Fig. 3 ).

In our analysis we have neglected the presence of baryons, in order to obtain simple analytical results. Baryons have two effects: They lead to $(\rho+3 p)_{\mathrm{rad}+\mathrm{bar}}>$ 0 , and therefore to an enhancement of the compression peaks (the first, third, etc. acoustic peak). In addition, the baryons slightly decrease the sound speed $c_{s}$, increasing thereby $\Delta k$ and decreasing $\Delta \vartheta$.

Another point which we have neglected is the fact that the universe became matter dominated at $\eta_{\text {eq }}$, only shortly before decoupling: $\eta_{\text {dec }} \simeq 4 \eta_{\text {eq }}$, for $\Omega_{m}=1$. As we have seen, the gravitational potential on sub-horizon scales is decaying in the radiation dominated era. If the radiation dominated era is not very long before decoupling, the gravitational potential is still slightly decaying and free streaming photons fall into a deeper gravitational potential than they have to climb out of. This effect, called "early integrated Sachs-Wolfe effect" adds to the photon temperature fluctuations at scales which are only slightly larger than the position of the first acoustic peak for adiabatic perturbations. It therefore 'boosts' this peak and, at the same time, moves it to lightly larger scales (larger angles, lower spherical harmonics). Since $\eta_{\text {eq }} \propto h^{-2}$, the first acoustic peak is larger if $h$ is smaller.

A small Hubble parameter increases therefore the acoustic peaks. A similar effect is observed if a cosmological constant or negative curvature are present, since $\eta_{\text {eq }}$ is retarded in those cases.

The real universe contains not only photons and dark matter, but also neutrinos and baryons. It has actually be found recently [20] that this 4-fluid mixture allows five 
different modes which grow or stay constant. The adiabatic mode, the dark matter isocurvature mode which we have just discussed, a similar baryon isocurvature mode and two neutrino isocurvature modes. The most generic initial conditions which allow for arbitrary correlations between the different modes are very unpredictable. We can maybe just say that they lead to a first acoustic peak in the range of $150 \leq \ell^{(2)} \leq 350$ for a spatially flat universe. In the rest of this review, we only discuss adiabatic perturbations, which are by far the most studied, but it is important to keep in mind that all the results especially concerning the estimation of cosmological parameters is not valid if we allow for more generic initial conditions $[1,2]$.

\section{Vector perturbations of perfect fluids}

$$
\text { If } \Pi^{(V)}=0 \text { equation }(2.56) \text { implies }
$$

$$
\Omega \propto a^{3 c_{s}^{2}-1}
$$

For $\dot{p} / \dot{\rho}=c_{s}^{2} \leq 1 / 3$, this leads to a non-growing vorticity. The dynamical Einstein equation implies

$$
\sigma^{(V)} \propto a^{-2}
$$

and the constraint (2.51) reads (at early times, so we can neglect curvature)

$$
\Omega \sim x^{2} \sigma^{(V)}
$$

If perturbations are created in the very early universe on super-horizon scales (e.g., during an inflationary period), vector perturbations of the metric decay and become soon entirely negligible. Even if $\Omega_{i}$ remains constant in a radiation dominated universe, it has to be so small on relevant scales at formation $\left(x_{i n} \ll 1\right)$ that we may safely neglect it.

\section{Tensor perturbations}

The situation is different for tensor perturbations. Again we consider the perfect fluid case, $\Pi_{i j}^{(T)}=0$. There (2.54) implies (if $\kappa$ is negligible)

$$
H_{i j}^{\prime \prime}+\frac{2 \beta}{x} H_{i j}^{\prime}+H_{i j}=0
$$

with $\beta=1$ in the radiation dominated era and $\beta=2$ in the matter dominated era. The less decaying mode solution to Eq. (3.65) is $H_{i j}=e_{i j} x^{1 / 2-\beta} J_{1 / 2-\beta}(x)$, where $J_{\nu}$ denotes the Bessel function of order $\nu$ and $e_{i j}$ is a transverse traceless polarization tensor. This leads to

$$
\begin{aligned}
& H_{i j}=\text { const } \quad \text { for } x \ll 1, \\
& H_{i j}=\frac{1}{a} \quad \text { for } x \gtrsim 1 .
\end{aligned}
$$

\section{CMB ANISOTROPIES}

\section{A. Light-like geodesics}

After decoupling, $\eta>\eta_{\text {dec }}$, photons follow to a good approximation light-like geodesics. The temperature shift is then given by the energy shift of a given photon.

The unperturbed photon trajectory follows $\left(x^{\mu}\right) \equiv$ $\left(\eta, \mathbf{n}\left(\eta-\eta_{0}\right)+\mathbf{x}_{0}\right)$, where $\mathbf{x}_{0}$ is the photon position at time $\eta_{0}$ and $\mathbf{n}$ is the (parallel transported) photon direction. With respect to a geodesic basis $(\mathbf{e})_{i=1}^{3}$, the components of $\mathbf{n}$ are constant. If $\kappa=0$ we may choose $\mathbf{e}_{i}=\partial / \partial x^{i}$; if $\kappa \neq 0$ these vector fields are no longer parallel transported and therefore do not form a geodesic basis $\left(\nabla_{\mathbf{e}_{i}} \mathbf{e}_{j}=0\right)$.

Our metric is of the form

$$
d \bar{s}^{2}=a^{2} d s^{2}
$$

with

$$
\begin{aligned}
& d s^{2}=\left(\gamma_{\mu \nu}+h_{\mu \nu}\right) d x^{\mu} d x^{\nu}, \\
& \gamma_{00}=-1, \gamma_{i 0}=0, \gamma_{i j}=\gamma_{j i}
\end{aligned}
$$

as before.

We make use of the fact that light-like geodesics are conformally invariant. More precisely $d s^{2}$ and $d \bar{s}^{2}$ have the same light-like geodesics, only the corresponding affine parameters are different. Let us denote the two affine parameters by $\lambda$ and $\bar{\lambda}$ respectively, and the tangent vectors to the geodesic by

$$
n=\frac{d x}{d \lambda}, \quad \bar{n}=\frac{d x}{d \bar{\lambda}}, \quad n^{2}=\bar{n}^{2}=0, \quad n^{0}=1, \quad \mathbf{n}^{2}=1 .
$$

We set $n^{0}=1+\delta n^{0}$. The geodesic equation for the perturbed metric

$$
d s^{2}=\left(\gamma_{\mu \nu}+h_{\mu \nu}\right) d x^{\mu} d x^{\nu}
$$

yields, to first order,

$$
\frac{d}{d \lambda} \delta n^{\mu}=-\delta \Gamma_{\alpha \beta}^{\mu} n^{\alpha} n^{\beta}
$$

For the energy shift, we have to determine $\delta n^{0}$. Since $g^{0 \mu}=-1 \cdot \delta_{0 \mu}+$ first order, we obtain $\delta \Gamma_{\alpha \beta}^{0}=$ $-1 / 2\left(h_{\alpha 0 \mid \beta}+h_{\beta 0 \mid \alpha}-\dot{h}_{\alpha \beta}\right)$, so that

$$
\frac{d}{d \lambda} \delta n^{0}=h_{\alpha 0 \mid \beta} n^{\beta} n^{\alpha}-\frac{1}{2} \dot{h}_{\alpha \beta} n^{\alpha} n^{\beta} .
$$

Integrating this equation we use $h_{\alpha 0 \mid \beta} n^{\beta}=\frac{d}{d \lambda}\left(h_{\alpha 0} n^{\alpha}\right)$, so that the change of $n^{0}$ between some initial time $\eta_{i}$ and some final time $\eta_{f}$ is given by

$$
\left.\delta n^{0}\right|_{i} ^{f}=\left[h_{00}+h_{0 j} n^{j}\right]_{i}^{f}-\frac{1}{2} \int_{i}^{f} \dot{h}_{\mu \nu} n^{\mu} n^{\nu} d \lambda .
$$


On the other hand, the ratio of the energy of a photon measured by some observer at $t_{f}$ to the energy emitted at $t_{i}$ is

$$
\frac{E_{f}}{E_{i}}=\frac{(\bar{n} \cdot u)_{f}}{(\bar{n} \cdot u)_{i}}=\frac{T_{f}}{T_{i}} \frac{(n \cdot u)_{f}}{(n \cdot u)_{i}}
$$

where $u_{f}$ and $u_{i}$ are the four-velocities of the observer and emitter respectively, and the factor $T_{f} / T_{i}$ is the usual (unperturbed) redshift, which relates $n$ and $\bar{n}$. The velocity field of observer and emitter is given by

$$
u=(1-A) \partial_{\eta}+v^{i} \partial_{i}
$$

An observer measuring a temperature $T_{0}$ receives photons that were emitted at the time $\eta_{\mathrm{dec}}$ of decoupling of matter and radiation, at the fixed temperature $T_{\text {dec }}$. In first-order perturbation theory, we find the following relation between the unperturbed temperatures $T_{f}, T_{i}$, the measurable temperatures $T_{0}, T_{\mathrm{dec}}$, and the photon density perturbation:

$$
\frac{T_{f}}{T_{i}}=\frac{T_{0}}{T_{\mathrm{dec}}}\left(1-\frac{\delta T_{f}}{T_{f}}+\frac{\delta T_{i}}{T_{i}}\right)=\frac{T_{0}}{T_{\mathrm{dec}}}\left(1-\left.\frac{1}{4} \delta^{(r)}\right|_{i} ^{f}\right),
$$

where $\delta^{(r)}$ is the intrinsic density perturbation in the radiation and we used $\rho^{(r)} \propto T^{4}$ in the last equality. Inserting the above equation and Eq. (4.7) into Eq. (4.8), and using Eq. (2.19) for the definition of $h_{\mu \nu}$, one finds, after integration by parts [6] the following result for scalar perturbations:

$$
\begin{aligned}
\frac{E_{f}}{E_{i}} & =\frac{T_{0}}{T_{\mathrm{dec}}}\left\{1-\left[\frac{1}{4} D_{g}^{(r)}+V_{j}^{(b)} n^{j}+\Psi-\Phi\right]_{i}^{f}\right. \\
& \left.+\int_{i}^{f}(\dot{\Psi}-\dot{\Phi}) d \lambda\right\} .
\end{aligned}
$$

Here $D_{g}^{(r)}$ denotes the density perturbation in the radiation fluid, and $V^{(b)}$ is the peculiar velocity of the baryonic matter component (the emitter and observer of radiation). The final time values in the square bracket of Eq. (4.11) give rise only to monopole contributions and to the dipole due to our motion with respect to the CMB, and will be neglected in what follows.

Evaluating Eq. (4.11) at final time $\eta_{0}$ (today) and initial time $\eta_{\text {dec }}$, we obtain the temperature difference of photons coming from different directions $\mathbf{n}$ and $\mathbf{n}^{\prime}$

$$
\frac{\Delta T}{T} \equiv \frac{\delta T(\mathbf{n})}{T}-\frac{\delta T\left(\mathbf{n}^{\prime}\right)}{T}
$$

with temperature perturbation

$$
\begin{aligned}
\frac{\Delta T(\mathbf{n})}{T} & =\left[\frac{1}{4} D_{g}^{(r)}+V_{j}^{(b)} n^{j}+\Psi-\Phi\right]\left(\eta_{\mathrm{dec}}, \mathbf{x}_{\mathrm{dec}}\right) \\
& +\int_{\eta_{\mathrm{dec}}}^{\eta_{\mathrm{o}}}(\dot{\Psi}-\dot{\Phi})(\eta, \mathbf{x}(\eta)) d \eta
\end{aligned}
$$

where $\mathbf{x}(\eta)$ is the unperturbed photon position at time $\eta$ for an observer at $\mathbf{x}_{0}$, and $\mathbf{x}_{\mathrm{dec}}=\mathbf{x}\left(\eta_{\mathrm{dec}}\right)$ (If $\kappa=0$ we simply have $\mathbf{x}(\eta)=\mathbf{x}_{0}-\left(\eta_{0}-\eta\right) \mathbf{n}$.). The first term in Eq. (4.13) describes the intrinsic inhomogeneities on the surface of last scattering, due to acoustic oscillations prior to decoupling. Depending on the initial conditions, it can contribute significantly on super-horizon scales. This is especially important in the case of adiabatic initial conditions. As we have seen in Eq. (3.44), in a dust + radiation universe with $\Omega=1$, adiabatic initial conditions imply $D_{g}^{(r)}(k, \eta)=-20 / 3 \Psi(k, \eta)$ and $V^{(b)}=V^{(r)} \ll D_{g}^{(r)}$ for $k \eta \ll 1$. With $\Phi=-\Psi$ the the square bracket of Eq. (4.13) gives

$$
\left(\frac{\Delta T(\mathbf{n})}{T}\right)_{\text {adiabatic }}^{(\mathrm{OSW})}=\frac{1}{3} \Psi\left(\eta_{\mathrm{dec}}, \mathbf{x}_{\mathrm{dec}}\right)
$$

on super-horizon scales. The contribution to $\frac{\delta T}{T}$ from the last scattering surface on very large scales is called the 'ordinary Sachs-Wolfe effect' (OSW). It has been derived for the first time by Sachs and Wolfe [27]. For isocurvature perturbations, the initial condition $D_{g}^{(r)}(k, \eta) \rightarrow 0$ for $\eta \rightarrow 0$ is satisfied and the contribution of $D_{g}$ to the ordinary Sachs-Wolfe effect can be neglected

$$
\left(\frac{\Delta T(\mathbf{n})}{T}\right)_{\text {isocurvature }}^{(\mathrm{OsW})}=2 \Psi\left(\eta_{\mathrm{dec}}, \mathbf{x}_{\mathrm{dec}}\right)
$$

The second term in (4.13) describes the relative motions of emitter and observer. This is the Doppler contribution to the CMB anisotropies. It appears on the same angular scales as the acoustic term, and we thus call the sum of the acoustic and Doppler contributions "acoustic peaks".

The last two terms are due to the inhomogeneities in the spacetime geometry; the first contribution determines the change in the photon energy due to the difference of the gravitational potential at the position of emitter and observer. Together with the part contained in $D_{g}^{(r)}$ they represent the "ordinary" Sachs-Wolfe effect. The integral accounts for red-shift or blue-shift caused by the time dependence of the gravitational field along the path of the photon, and represents the so-called integrated Sachs-Wolfe (ISW) effect. In a $\Omega=1$, pure dust universe, the Bardeen potentials are constant and there is no integrated Sachs-Wolfe effect; the blue-shift which the photons acquire by falling into a gravitational potential is exactly canceled by the redshift induced by climbing out of it. This is no longer true in a universe with substantial radiation contribution, curvature or a cosmological constant.

The sum of the ordinary Sachs-Wolfe term and the integral is the full Sachs-Wolfe contribution (SW).

For vector perturbations $\delta^{(r)}$ and $A$ vanish and Eq. (4.8) leads to

$$
\begin{aligned}
\left(E_{f} / E_{i}\right)^{(V)} & =\left(a_{i} / a_{f}\right)\left[1-\left.V_{j}^{(m)} n^{j}\right|_{i} ^{f}\right. \\
& \left.+\int_{i}^{f} \dot{\sigma}_{j} n^{j} d \lambda\right] .
\end{aligned}
$$




\section{R. DURRER}

We obtain a Doppler term and a gravitational contribution. For tensor perturbations, i.e., gravitational waves, only the gravitational part remains:

$$
\left(E_{f} / E_{i}\right)^{(T)}=\left(a_{i} / a_{f}\right)\left[1-\int_{i}^{f} \dot{H}_{l j} n^{l} n^{j} d \lambda\right] .
$$

Equations (4.11), (4.14) and (4.15) are the manifestly gauge invariant results for the Sachs-Wolfe effect for scalar vector and tensor perturbations. Disregarding again the dipole contribution due to our proper motion, Eqs. (4.14), (4.15) imply the vector and tensor temperature fluctuations

$$
\begin{aligned}
\left(\frac{\Delta T(\mathbf{n})}{T}\right)^{(V)} & =V_{j}^{(m)}\left(\eta_{\mathrm{dec}}, \mathbf{x}_{\mathrm{dec}}\right) n^{j} \\
& +\int_{i}^{f} \dot{\sigma}_{j}(\eta, \mathbf{x}(\eta)) n^{j} d \lambda, \\
\left(\frac{\Delta T(\mathbf{n})}{T}\right)^{(T)} & =-\int_{i}^{f} \dot{H}_{l j}(\eta, \mathbf{x}(\eta)) n^{l} n^{j} d \lambda .
\end{aligned}
$$

Note that for models where initial fluctuations have been led down in the very early universe, vector perturbations are irrelevant as we have already pointed out. In this sense Eq. (4.16) is here mainly for completeness. However, in models where perturbations are sourced by some inherently inhomogeneous component (e.g., topological defects) vector perturbation can be important.

\section{B. Power spectra}

One of the basic tools to compare models of large scale structure with observations are power spectra. They are the "harmonic transforms" of the two point correlation functions. If the perturbations of the model under consideration are Gaussian (a relatively generic prediction from inflationary models), then the power spectra contain the full statistical information of the model.

One important power spectrum is the dark matter power spectrum,

$$
P_{D}(k)=\left\langle\left|D_{g}^{(m)}\left(\mathbf{k}, \eta_{0}\right)\right|^{2}\right\rangle
$$

where \langle\rangle indicates a statistical average over "initial conditions" in a given model. $P_{D}(k)$ is usually compared with the observed power spectrum of the galaxy distribution.
Another power spectrum is given by the velocity perturbations,

$$
P_{V}(k)=\left\langle\left|\mathbf{V}\left(\mathbf{k}, \eta_{0}\right)\right|^{2}\right\rangle \simeq H_{0}^{2} \Omega^{1.2} P_{D}(k) k^{-2} .
$$

For $\simeq$ we have used that $|k V|\left(\eta_{0}\right)=\dot{D}_{g}^{(m)}\left(\eta_{0}\right) \sim$ $H_{0} \Omega^{0.6} D_{g}$ on sub-horizon scales (see, e.g., [15]).

The power spectrum we are most interested in is the CMB anisotropy power spectrum. It is defined as follows: $\Delta T / T$ is a function of position $\mathbf{x}_{0}$, time $\eta_{0}$ and photon direction $\mathbf{n}$. We develop the $\mathbf{n}$-dependence in terms of spherical harmonics. We will suppress the argument $\eta_{0}$ and often also $\mathbf{x}_{0}$ in the following calculations. All results are for today $\left(\eta_{0}\right)$ and here $\left(\mathbf{x}_{0}\right)$. By statistical homogeneity expectation values are supposed to be independent of position. Furthermore, we assume that the process generating the initial perturbations is statistically isotropic. Then, the off-diagonal correlators of the expansion coefficients $a_{\ell m}$ vanish and we have

$$
\begin{aligned}
& \frac{\Delta T}{T}\left(\mathbf{x}_{0}, \mathbf{n}, \eta_{0}\right)=\sum_{\ell, m} a_{\ell m}\left(\mathbf{x}_{0}\right) Y_{\ell m}(\mathbf{n}), \\
& \left\langle a_{\ell m} \cdot a_{\ell^{\prime} m^{\prime}}^{*}\right\rangle=\delta_{\ell \ell^{\prime}} \delta_{m m^{\prime}} C_{\ell} .
\end{aligned}
$$

The $C_{\ell}$ 's are the CMB power spectrum. We assume that the perturbations are generated by a homogeneous and isotropic process, so that $C_{\ell}$ depends neither on $\mathbf{x}_{0}$ nor on $m$, and that $\left\langle a_{\ell m} \cdot a_{\ell^{\prime} m^{\prime}}^{*}\right\rangle$ vanishes for $\ell \neq \ell^{\prime}$ or $m \neq m^{\prime}$.

Let us, at this point insert a comment on the problem of cosmic variance: Even if our 'ergodic hypothesis' is correct and we may interchange ensemble and spatial averages, we cannot obtain very precise averages for measurements of large scale characteristics, due to the fact that we can observe only the universe around a given position. For example, let us assume that temperature fluctuations are Gaussian, as they are in most inflationary models. The functions $a_{\ell m}$ are then also Gaussian distributed, and we have a variance of

$$
\left.\left|\frac{1}{2 \ell+1} \sum_{m=-\ell}^{\ell}\right| a_{\ell m}\right|^{2}-C_{\ell}|=| C_{\ell}^{\mathrm{obs}}-C_{\ell} \mid=\frac{C_{\ell}}{2 \ell+1},
$$

on the average of the $2 \ell+1$ values $a_{\ell m}$ which can in principle be measured from one point with full sky coverage. For simplicity, we neglect the increase of the variance due to the fact that our own Milky Way blocks a portion of sky of about $20 \%$. Wick's theorem now gives

$$
\left\langle C_{\ell}^{2}\right\rangle-\left\langle C_{\ell}\right\rangle^{2}=\left\langle\left|a_{\ell m}\right|^{4}\right\rangle-\left\langle\left|a_{\ell m}\right|^{2}\right\rangle^{2}=2\left\langle\left|a_{\ell m}\right|^{2}\right\rangle^{2}
$$

For a given multipole $\ell$ we then expect a variance of the $C_{\ell}$ 's

$$
\frac{\sqrt{\left(C_{\ell}^{\text {obs }}\right)^{2}-C_{\ell}^{2}}}{C_{\ell}}=\sqrt{\frac{2}{2 \ell+1}},
$$

in real experiments, this 'cosmic variance' is in general much larger due to the limited sky coverage. 
The two point correlation function is related to the $C_{\ell}$ 's by

$$
\begin{aligned}
\left\langle\frac{\Delta T}{T}(\mathbf{n}) \frac{\Delta T}{T}\left(\mathbf{n}^{\prime}\right)\right\rangle_{\mathbf{n} \cdot \mathbf{n}^{\prime}=\mu} & =\sum_{\ell, \ell^{\prime}, m, m^{\prime}}\left\langle a_{\ell m} \cdot a_{\ell^{\prime} m^{\prime}}^{*}\right\rangle Y_{\ell m}(\mathbf{n}) Y_{\ell^{\prime} m^{\prime}}^{*}\left(\mathbf{n}^{\prime}\right) \\
& =\sum_{\ell} C_{\ell} \underbrace{\sum_{m=-\ell}^{\ell} Y_{\ell m}(\mathbf{n}) Y_{\ell m}^{*}\left(\mathbf{n}^{\prime}\right)}_{\frac{2 \ell+1}{4 \pi} P_{\ell}\left(\mathbf{n} \cdot \mathbf{n}^{\prime}\right)}=\frac{1}{4 \pi} \sum_{\ell}(2 \ell+1) C_{\ell} P_{\ell}(\mu),
\end{aligned}
$$

where we have used the addition theorem of spherical harmonics for the last equality. The $P_{\ell}$ 's are the Legendre polynomials.

Clearly the $a_{l m}$ 's from scalar, vector and tensor perturbations are uncorrelated,

$$
\left\langle a_{\ell m}^{(S)} a_{\ell^{\prime} m^{\prime}}^{(V)}\right\rangle=\left\langle a_{\ell m}^{(S)} a_{\ell^{\prime} m^{\prime}}^{(T)}\right\rangle=\left\langle a_{\ell m}^{(V)} a_{\ell^{\prime} m^{\prime}}^{(T)}\right\rangle=0
$$

Since vector perturbations decay, their contributions, the $C_{\ell}^{(V)}$, are negligible in models where initial perturbations have been laid down very early, e.g., after an inflationary period. Tensor perturbations are constant on super-horizon scales and perform damped oscillations once they enter the horizon.

Let us first discuss in somewhat more detail scalar perturbations. We specialize to the case $\kappa=0$ for simplicity. We suppose the initial perturbations to be given by a spectrum,

$$
\left\langle|\Psi|^{2}\right\rangle k^{3}=A^{2} k^{n-1} \eta_{0}^{n-1}
$$

We multiply by the constant $\eta_{0}^{n-1}$, the actual comoving size of the horizon, in order to keep $A$ dimensionless for all values of $n$. $A$ then represents the amplitude of metric perturbations at horizon scale today, $k=1 / \eta_{0}$.

On super-horizon scales we have, for adiabatic perturbations:

$$
\frac{1}{4} D_{g}^{(r)}=-\frac{5}{3} \Psi+\mathcal{O}\left(x^{2}\right), \quad V^{(b)}=V^{(r)}=\mathcal{O}(x)
$$

The dominant contribution on super-horizon scales (neglecting the integrated Sachs-Wolfe effect $\int \dot{\Phi}-\dot{\Psi}$ ) is then

$$
\frac{\Delta T}{T}\left(\mathbf{x}_{0}, \mathbf{n}, \eta_{0}\right)=\frac{1}{3} \Psi\left(x_{\mathrm{dec}}, \eta_{\mathrm{dec}}\right)
$$

The Fourier transform of (4.26) gives

$$
\frac{\Delta T}{T}\left(\mathbf{k}, \mathbf{n}, \eta_{0}\right)=\frac{1}{3} \Psi\left(k, \eta_{\mathrm{dec}}\right) \cdot e^{i \mathbf{k n}\left(\eta_{\mathrm{o}}-\eta_{\mathrm{dec}}\right)} .
$$

Using the decomposition

$$
e^{i \mathbf{k n}\left(\eta_{0}-\eta_{\mathrm{dec}}\right)}=\sum_{\ell=0}^{\infty}(2 \ell+1) i^{\ell} j_{\ell}\left(k\left(\eta_{0}-\eta_{\mathrm{dec}}\right)\right) P_{\ell}(\widehat{\mathbf{k}} \cdot \mathbf{n})
$$

where $j_{\ell}$ are the spherical Bessel functions, we obtain

$$
\begin{aligned}
& \left\langle\frac{\Delta T}{T}\left(\mathbf{x}_{0}, \mathbf{n}, \eta_{0}\right) \frac{\Delta T}{T}\left(\mathbf{x}_{0}, \mathbf{n}^{\prime}, \eta_{0}\right)\right\rangle=\frac{1}{V} \int d^{3} x_{0}\left\langle\frac{\Delta T}{T}\left(\mathbf{x}_{0}, \mathbf{n}, \eta_{0}\right) \frac{\Delta T}{T}\left(\mathbf{x}_{0}, \mathbf{n}^{\prime}, \eta_{0}\right)\right\rangle \\
& =\frac{1}{(2 \pi)^{3}} \int d^{3} k\left\langle\frac{\Delta T}{T}\left(\mathbf{k}, \mathbf{n}, \eta_{0}\right)\left(\frac{\Delta T}{T}\right)^{*}\left(\mathbf{k}, \mathbf{n}^{\prime}, \eta_{0}\right)\right\rangle \\
& =\frac{1}{(2 \pi)^{3}} \int d^{3} k\left\langle|\Psi|^{2}\right\rangle \sum_{\ell, \ell^{\prime}=0}^{\infty}(2 \ell+1)\left(2 \ell^{\prime}+1\right) j_{\ell}\left(k\left(\eta_{0}-\eta_{\mathrm{dec}}\right)\right) j_{\ell^{\prime}}\left(k\left(\eta_{0}-\eta_{\mathrm{dec}}\right)\right) i^{\ell-\ell^{\prime}} P_{\ell}(\hat{\mathbf{k}} \cdot \mathbf{n}) P_{\ell^{\prime}}\left(\hat{\mathbf{k}} \cdot \mathbf{n}^{\prime}\right)
\end{aligned}
$$

Inserting $P_{\ell}(\hat{\mathbf{k n}})=\frac{4 \pi}{2 \ell+1} \sum_{m} Y_{\ell m}^{*}(\hat{\mathbf{k}}) Y_{\ell m}(\mathbf{n})$ and $P_{\ell^{\prime}}\left(\hat{\mathbf{k}} \mathbf{n}^{\prime}\right)=\frac{4 \pi}{2 \ell^{\prime}+1} \sum_{m^{\prime}} Y_{\ell^{\prime} m^{\prime}}^{*}(\hat{\mathbf{k}}) Y_{\ell^{\prime} m^{\prime}}\left(\mathbf{n}^{\prime}\right)$, integration over the directions $d \Omega_{\hat{k}}$ gives $\delta_{\ell \ell^{\prime}} \delta_{m m^{\prime}} \sum_{m} Y_{\ell m}^{*}(\mathbf{n}) Y_{\ell m}\left(\mathbf{n}^{\prime}\right)$. Using as well $\sum_{m} Y_{\ell m}^{*}(\mathbf{n}) Y_{\ell m}\left(\mathbf{n}^{\prime}\right)=\frac{2 \ell+1}{4 \pi} P_{\ell}(\mu)$, where $\mu=\mathbf{n} \cdot \mathbf{n}^{\prime}$, we find 


\section{R. DURRER}

$$
\left\langle\frac{\Delta T}{T}\left(\mathbf{x}_{0}, \mathbf{n}, \eta_{0}\right) \frac{\Delta T}{T}\left(\mathbf{x}_{0}, \mathbf{n}^{\prime}, \eta_{0}\right)\right\rangle_{\mathbf{n n}^{\prime}=\mu}=\sum_{\ell} \frac{2 \ell+1}{4 \pi} P_{\ell}(\mu) \frac{2}{\pi} \int \frac{d k}{k}\left\langle\frac{1}{9}|\Psi|^{2}\right\rangle k^{3} j_{\ell}^{2}\left(k\left(\eta_{0}-\eta_{\mathrm{dec}}\right)\right) .
$$

Comparing this equation with Eq. (4.22) we obtain for adiabatic perturbations on scales $2 \leq \ell \ll \chi\left(\eta_{0}-\eta_{\text {dec }}\right) / \eta_{\text {dec }}$ $\sim 100$

$$
C_{\ell}^{(\mathrm{SW})} \simeq C_{\ell}^{(\mathrm{OSW})} \simeq \frac{2}{\pi} \int_{0}^{\infty} \frac{d k}{k}\left\langle\left|\frac{1}{3} \Psi\right|^{2}\right\rangle k^{3} j_{\ell}^{2}\left(k\left(\eta_{0}-\eta_{\mathrm{dec}}\right)\right)
$$

If $\Psi$ is a pure power law and we set $k\left(\eta_{0}-\eta_{\text {dec }}\right) \sim k \eta_{0}$, the integral $(4.30)$ can be performed analytically. For the ansatz (4.24) one finds for $-3<n<3$

$$
C_{\ell}^{(\mathrm{SW})}=\frac{A^{2}}{9} \frac{\Gamma(3-n) \Gamma\left(\ell-\frac{1}{2}+\frac{n}{2}\right)}{2^{3-n} \Gamma^{2}\left(2-\frac{n}{2}\right) \Gamma\left(\ell+\frac{5}{2}-\frac{n}{2}\right)} .
$$

Of special interest is the scale invariant spectrum, $n=1$. This spectrum with a time and scale independent gravitational potential has first been investigated by Harrison and by Zel'dovich [29]. It is therefore called the Harrison-Zel'dovich spectrum. It leads to

$$
\ell(\ell+1) C_{\ell}^{(\mathrm{SW})}=\mathrm{const} \simeq\left\langle\left(\frac{\Delta T}{T}\left(\vartheta_{\ell}\right)\right)^{2}\right\rangle, \quad \vartheta_{\ell} \equiv \pi / \ell .
$$

This is precisely (within the accuracy of the experiment) the behavior observed by the DMR experiment aboard COBE [8].

Inflationary models predict very generically a HZ spectrum (up to small corrections). The DMR discovery has therefore been regarded as a great success, if not a proof, of inflation. There are however other models like topological defects [31-33] or certain string cosmology models [34] which also predict scale-invariant, i.e., Harrison Zel'dovich spectra of fluctuations. These models do however not belong to the class investigated here, since in these models perturbations are induced by seeds which evolve non-linearly in time.

For isocurvature perturbations, the main contribution on large scales comes from the integrated Sachs-Wolfe effect and (4.30) is replaced by

$$
C_{\ell}^{(\mathrm{ISW})} \simeq \frac{8}{\pi} \int \frac{d k}{k} k^{3}\left\langle\left|\int_{\eta_{\mathrm{dec}}}^{\eta_{0}} \dot{\Psi}(k, \eta) j_{\ell}^{2}\left(k\left(\eta_{0}-\eta\right)\right) d \eta\right|^{2}\right\rangle .
$$

Inside the horizon $\Psi$ is roughly constant (matter dominated). Using the ansatz (4.24) for $\Psi$ inside the horizon and setting the integral in $(4.33) \sim 2 \Psi(k, \eta=1 / k) j_{\ell}^{2}\left(k \eta_{0}\right)$, we obtain again (4.31), but with $A^{2} / 9$ replaced by $4 A^{2}$. The Sachs-Wolfe temperature anisotropies coming from isocurvature perturbations are therefore about a factor of 6 times larger than those coming from adiabatic perturbations.

On smaller scales, $\ell \gtrsim 100$ the contribution to $\Delta T / T$ is usually dominated by acoustic oscillations, the first two terms in Eq. (4.13). Instead of (4.33) we then obtain

$$
C_{\ell}^{(\mathrm{AC})} \simeq \frac{2}{\pi} \int_{0}^{\infty} \frac{d k}{k} k^{3}\left\langle\left|\frac{1}{4} D_{g}^{(r)}\left(k, \eta_{\mathrm{dec}}\right) j_{\ell}\left(k \eta_{0}\right)+V^{(r)}\left(k, \eta_{\mathrm{dec}}\right) j_{\ell}^{\prime}\left(k \eta_{0}\right)\right|^{2}\right\rangle .
$$

On sub-horizon scales $D_{g}^{(r)}$ and $V^{(r)}$ are oscillating like sine or cosine waves depending on the initial conditions. Correspondingly the $C_{\ell}^{(A C)}$ will show peaks and minima. On very small scales they are damped by the photon diffusion which takes place during the recombination process (see next section).

For gravitational waves (tensor fluctuations), a formula analogous to (4.31) can be derived (see appendix),

$$
C_{\ell}^{(T)}=\frac{2}{\pi} \int d k k^{2}\left\langle\left|\int_{\eta_{\mathrm{dec}}}^{\eta_{0}} d \eta \dot{H}(\eta, k) \frac{j_{\ell}\left(k\left(\eta_{0}-\eta\right)\right)}{\left(k\left(\eta_{0}-\eta\right)\right)^{2}}\right|^{2}\right\rangle \frac{(\ell+2) !}{(\ell-2) !} .
$$

To a very crude approximation we may assume $\dot{H}=0$ on super-horizon scales and $\int d \eta \dot{H} j_{\ell}\left(k\left(\eta_{0}-\eta\right)\right) \sim H(\eta=$ $1 / k) j_{\ell}\left(k \eta_{0}\right)$. For a pure power law,

$$
k^{3}\left\langle|H(k, \eta=1 / k)|^{2}\right\rangle=A_{T}^{2} k^{n_{T}} \eta_{0}^{-n_{T}},
$$






Fig. 5. A COBE normalized sample adiabatic (solid line) and isocurvature (dashed line) CMB anisotropy spectrum, $\ell(\ell+1) C_{\ell}$, are shown on the top panel. The quantity shown in the bottom panel is the ratio of temperature fluctuations for fixed value of $A$ (from Kanazawa et al. [35]).

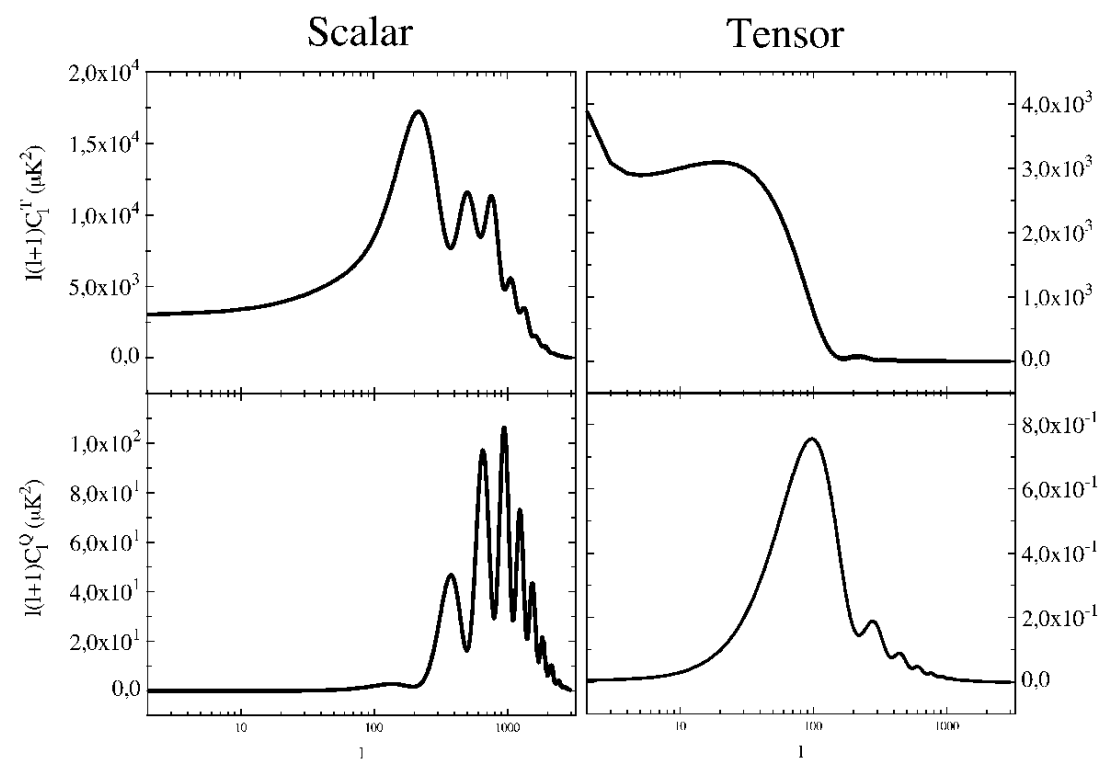

Fig. 6. Adiabatic scalar and tensor CMB anisotropy spectra are shown (top panels). The bottom panels show the corresponding polarization spectra (see Section IV.D). (from [25]).

this gives

$$
C_{\ell}^{(T)} \simeq \frac{2}{\pi} \frac{(\ell+2) !}{(\ell-2) !} A_{T}^{2} \int \frac{d x}{x} x^{n_{T}} \frac{j_{\ell}^{2}(x)}{x^{4}}=\frac{(\ell+2) !}{(\ell-2) !} A_{T}^{2} \frac{\Gamma\left(6-n_{T}\right) \Gamma\left(\ell-2+\frac{n_{T}}{2}\right)}{2^{6-n_{T}} \Gamma^{2}\left(\frac{7}{2}-n_{T}\right) \Gamma\left(\ell+4-\frac{n_{T}}{2}\right)} .
$$




\section{R. DURRER}

For a scale invariant spectrum $\left(n_{T}=0\right)$ this results in

$$
\ell(\ell+1) C_{\ell}^{(T)} \simeq \frac{\ell(\ell+1)}{(\ell+3)(\ell-2)} A_{T}^{2} \frac{8}{15 \pi} .
$$

The singularity at $\ell=2$ in this crude approximation is not real, but there is some enhancement of $\ell(\ell+1) C_{\ell}^{(T)}$ at $\ell \sim 2$.

Since tensor perturbations decay on sub-horizon scales, $\ell \gtrsim 60$, they are not very sensitive to cosmological parameters.

Again, inflationary models (and topological defects) predict a scale invariant spectrum of tensor fluctuations $\left(n_{T} \sim 0\right)$.

On very small angular scales, $\ell \geq 800$, fluctuations are damped by collisional damping (Silk damping). This effect has to be discussed with the Boltzmann equation for photons derived in the next section.

\section{The Boltzmann equation}

\section{Elements of the derivation}

When particles are not very tightly coupled, the fluid approximation breaks down and they have to be described by a Boltzmann equation,

$$
p^{\mu} \partial_{\mu} f-\Gamma_{\alpha \beta}^{i} p^{\alpha} p^{\beta} \frac{\partial f}{\partial p^{i}}=C[f] .
$$

$C[f]$ is a collision integral which describes the interactions with other matter components. The left hand side of (4.39) just requires the particles to move along geodesics in the absence of collisions.

Let us first consider the situation where collisions are negligible, $C[f]=0$. The unperturbed Boltzmann equation implies that $f$ be a function of $v=a p$ only. Setting $f=\bar{f}(v)+F(\eta, \mathbf{x}, v, \mathbf{n})$, where $\mathbf{n}$ denotes the momentum directions, leads then to the perturbation equation

$$
\begin{aligned}
\partial_{\eta} F- & n^{i} \partial_{i} F-\Gamma_{j k}^{(S) i} n^{j} n^{k} \frac{\partial F}{\partial n^{i}} \\
= & v \frac{d \bar{f}}{d v}\left[n^{i} A_{, i}-n^{i} n^{j}\left(B_{i \mid j}-\dot{H}_{i j}\right)+H_{L}\right] .
\end{aligned}
$$

Here $\Gamma_{j k}^{(S) i}$ are the Christoffel symbols of the space of constant curvature $\kappa$.

To derive $(4.40)$, we have used $p^{2}=0$. The Liouville equation for particles with non-vanishing mass can be found in Ref. [6].

The ansatz

$$
f(x, \mathbf{p})=\bar{f}\left(\frac{g^{(3)}(\mathbf{p}, \mathbf{p})^{\frac{1}{2}}}{T(x, \mathbf{n})}\right)=\bar{f}\left(\frac{\bar{T} v}{T(x, \mathbf{n})}\right)
$$

with $T(x, \mathbf{n})=\bar{T}(\eta)+\Delta T(x, \mathbf{n})$ leads to

$$
f=\bar{f}-v \frac{d \bar{f}}{d v} \frac{\Delta T}{\bar{T}}
$$

Integrating (4.40) over photon energies, we can also write

$$
\frac{\Delta T}{T}=\frac{1}{4} \imath
$$

where $\imath$ is the brightness perturbation,

$$
\imath=\frac{4 \pi}{\bar{\rho} a^{4}} \int_{0}^{\infty} F v^{3} d v
$$

Setting $F=-v \frac{d \bar{f}}{d v} \frac{\Delta T}{T}$, we find

$$
\begin{aligned}
\partial_{\eta}\left(\frac{\Delta T}{T}\right) & +n^{i} \partial_{i}\left(\frac{\Delta T}{T}\right)-\Gamma_{j k}^{(S) i} n^{j} n^{k} \frac{\partial\left(\frac{\Delta T}{T}\right)}{\partial n^{i}} \\
& =-\left[n^{i} A, i-\left(B_{i \mid j}-\dot{H}_{i j}\right) n^{i} n^{j}+H_{L}\right]
\end{aligned}
$$

The fact that gravitational perturbations of Liouville's equation can be cast purely in temperature perturbations of the original distribution is not astonishing. This is just an expression of gravity being "achromatic", i.e., independent of the photon energy.

We now decompose (4.45) into scalar, vector and tensor components. Even though $\Delta T / T$ is just a function, it can be represented in the form

$$
\frac{\Delta T}{T}(\mathbf{x}, \mathbf{n})=\sum_{\ell=0}^{\infty} \alpha_{i_{1}, \ldots, i_{\ell}}(\mathbf{x}) n^{i_{1}} \cdots n^{i_{\ell}}
$$

where the $\alpha_{i_{1}, \ldots, i_{\ell}}$ are symmetric traceless tensor fields that contain scalar, vector, 2-tensor and in principle also higher tensor components. Since spin components larger than 2 are not sourced by the right hand side of equation (4.46) and since they are suppressed at early times, when collisions are important, we neglect them here.

For the scalar contribution to $\Delta T / T$ we obtain from (4.46)

$$
\begin{aligned}
& \partial_{\eta}\left(\frac{\Delta T}{T}\right)^{(S)}+k \mu\left(\frac{\Delta T}{T}\right)^{(S)}-\Gamma_{j k}^{(S) i} n^{j} n^{k} \frac{\partial\left(\frac{\Delta T}{T}\right)^{(S)}}{\partial n^{i}} \\
& =-\left[k \mu A+\mu^{2} k^{2}\left(B-\dot{H}_{T}\right)+H_{L}+\frac{1}{3} k^{2} \dot{H}_{T}\right],
\end{aligned}
$$

where we have introduced the "direction cosine" $\mu$ defined by $n^{i} Y,_{i}=k \mu Y$. Note that in flat space, $\kappa=0$, we have just $\mu=i \hat{\boldsymbol{k}} \cdot \boldsymbol{n}$.

This equation is not manifestly gauge-invariant. However, setting

$$
\mathcal{M}^{(S)}=\left(\frac{\Delta T}{T}\right)^{(S)}+H_{L}+\frac{1}{3} k^{2} H_{T}+k \mu(\dot{H}-B),
$$

it reduces to

$$
\partial_{\eta} \mathcal{M}^{(S)}+k \mu \mathcal{M}^{(S)}-\Gamma_{j k}^{(S) i} n^{j} n^{k} \frac{\partial \mathcal{M}^{(S)}}{\partial n^{i}}=k \mu(\Phi-\Psi),
$$


where $\Phi$ and $\Psi$ are the Bardeen potentials. If $n^{j}$ are components with respect to a geodesic basis (or $\kappa=0$ ), the third term on the left hand side of Eq. (4.49) vanishes. For simplicity we now concentrate on the case $\kappa=0$. We can then integrate the equation and obtain

$$
\begin{aligned}
& \mathcal{M}^{(S)}\left(\eta_{0}, \mathbf{n}, \mathbf{k}\right)=\exp \left[i \mathbf{k} \cdot \mathbf{n}\left(\eta_{\text {in }}-\eta_{0}\right)\right] \mathcal{M}^{(S)}\left(\eta_{\text {in }}, \mathbf{n}, \mathbf{k}\right) \\
& +\int_{\eta_{\text {in }}}^{\eta_{0}} i \exp \left[i \mathbf{k} \cdot \mathbf{n}\left(\eta-\eta_{0}\right)\right] \mathbf{n} \cdot \mathbf{k}(\Phi-\Psi) d \eta
\end{aligned}
$$

Integration by parts and neglecting the monopole term $(\Phi-\Psi)\left(\eta_{0}\right)$, leads to

$$
\begin{aligned}
\mathcal{M}^{(S)}( & \left.\eta_{0}, \mathbf{n}, \mathbf{k}\right)=\exp \left[i \mathbf{k} \cdot \mathbf{n}\left(\eta_{\text {in }}-\eta_{0}\right)\right] \\
& \times\left[\mathcal{M}^{(S)}\left(\eta_{\text {in }}, \mathbf{n}, \mathbf{k}\right)+(\Phi-\Psi)\left(\eta_{\text {in }}, \mathbf{k}\right)\right] \\
& -\int_{\eta_{\text {in }}}^{\eta_{0}} \exp \left[i \mathbf{k} \cdot \mathbf{n}\left(\eta-\eta_{0}\right)\right](\dot{\Phi}-\dot{\Psi}) d \eta
\end{aligned}
$$

Comparing this equation with (4.13), we see again that $\mathcal{M}^{(S)}=\left(\frac{\Delta T}{T}\right)^{(S)}$ (up to gauge dependent monopole and dipole contributions) if the initial condition is

$$
\mathcal{M}^{(S)}\left(\eta_{\text {in }}, \mathbf{n}, \mathbf{k}\right)=\frac{1}{4} D_{g}^{(r)}\left(\eta_{\text {in }}, \mathbf{k}\right)+\mathbf{n} \cdot \mathbf{k} V^{(b)}\left(\eta_{\text {in }}, \mathbf{k}\right),
$$

which is equivalent to require that $\mathcal{M}^{(S)}\left(\eta_{\text {in }}\right)$ has no higher than first moments. As we shall see below, this assumption is quite reasonable since collisions suppress the build up of higher moments before recombination.

Since the right hand side of (4.49) is gauge invariant, the left hand side must be so as well and we conclude that $\mathcal{M}^{(S)}$ is a gauge-invariant variable (a direct proof of this, analyzing the gauge transformation properties of the distribution function, can be found in Ref. [6]).

$\mathcal{M}^{(S)}$ used in this work coincides with the scalar temperature fluctuations up a to a gauge dependent monopole and dipole contribution. In other work [48] the gauge invariant variable $\Theta \equiv \mathcal{M}^{(S)}-\Phi$ has been used. Since $\Phi$ is independent of the photon direction $\mathbf{n}$ this difference in the definition shows up only in the monopole, $C_{0}$.

The vector and tensor parts of $\Delta T / T$ are gaugeinvariant by themselves and we denote them by $\mathcal{M}^{(V)}$ and $\mathcal{M}^{(T)}$ for consistency. In the absence of collisions and with vanishing spatial curvature, they satisfy the equations

$$
\begin{aligned}
& \dot{\mathcal{M}}^{(V)}+i \mu k \mathcal{M}^{(V)}=-i n^{\ell} n^{m} k_{\ell} \sigma_{m}^{(V)} \\
& \dot{\mathcal{M}}^{(T)}+i \mu k \mathcal{M}^{(T)}=-i n^{\ell} n^{m} \dot{H}_{m \ell}
\end{aligned}
$$

The components of the energy momentum tensor are obtained by integrating the second moments of the distribution function over the mass shell,

$$
T^{\mu \nu}=\int_{P_{m}(x)} p^{\mu} p^{\nu} f(p, x) \frac{p^{2} d p d \Omega_{\hat{\mathrm{p}}}}{p^{0}},
$$

where $\Omega_{\hat{\mathrm{p}}}$ denotes the angular integration over momentum directions. One finds for $\kappa=0$ and setting $\mu=\mathbf{n} \cdot \hat{\mathbf{k}}$ :

$$
\begin{aligned}
D_{g}^{(r)} & =\frac{1}{\pi} \int \mathcal{M}^{(S)} d \Omega, \\
V^{(r)} & =\frac{3 i}{4 \pi} \int \mu \mathcal{M}^{(S)} d \Omega, \\
\Pi^{(r)} & =\frac{9}{2 \pi} \int\left(\mu^{2}-\frac{1}{3}\right) \mathcal{M}^{(S)} d \Omega, \\
\Gamma^{(r)} & =0, \\
V_{i}^{(V)} & =\frac{1}{4 \pi} \int n_{i} \mathcal{M}^{(V)} d \Omega, \\
\Pi_{j}^{(V)} & =\frac{6}{\pi} \int \mu n_{j} \mathcal{M}^{(V)} d \Omega, \\
\Pi_{i j}^{(T)} & =\frac{3}{\pi} \int n_{i} n_{j} \mathcal{M}^{(T)} d \Omega .
\end{aligned}
$$

Let us now turn to the collision term. At recombination (when the fluid description of radiation breaks down) the temperature is $\sim 0.4 \mathrm{eV}$. The electrons and nuclei are non-relativistic and the dominant collision process is non-relativistic Thomson scattering. Since collisions are important only before and during recombination, where curvature effects are entirely negligible, we set $\kappa=0$ in the reminder of this section.

The collision term is given by

$$
C[f]=\frac{d f_{+}}{d \eta}-\frac{d f_{-}}{d \eta},
$$

where $f_{+}$and $f_{-}$denote the distribution of photons scattered into respectively out of the beam due to Compton scattering.

In the matter (baryon/electron) rest frame, which we indicate by a prime, we know

$$
\frac{d f_{+}^{\prime}}{d t^{\prime}}(p, \mathbf{n})=\frac{\sigma_{T} n_{e}}{4 \pi} \int f^{\prime}\left(p^{\prime}, \mathbf{n}^{\prime}\right) \omega\left(\mathbf{n}, \mathbf{n}^{\prime}\right) d \Omega^{\prime},
$$

where $n_{e}$ denotes the number density of free electrons, $\sigma_{T}$ is the Thomson cross section, and $\omega$ is the normalized angular dependence of the Thomson cross section:

$$
\omega\left(\mathbf{n}, \mathbf{n}^{\prime}\right)=3 / 4\left[1+\left(\mathbf{n} \cdot \mathbf{n}^{\prime}\right)^{2}\right]=1+\frac{3}{4} n_{i j} n_{i j}^{\prime}
$$

with

$$
n_{i j} \equiv n_{i} n_{j}-\frac{1}{3} \delta_{i j}
$$

In the baryon rest frame which moves with four velocity $u$, the photon energy is given by

$$
p^{\prime}=p_{\mu} u^{\mu} \text {. }
$$

We denote by $p$ the photon energy with respect to a tetrad adapted to the slicing of spacetime into $\{\eta=$ constant\} hyper-surfaces:

$$
p=p_{\mu} n^{\mu}, \quad \text { with } \quad n=a^{-1}\left[(1-A) \partial_{\eta}+B^{i} \partial_{i}\right] .
$$

The unit vector $n$ is the normal to the hyper-surfaces of constant time. The lapse function and the shift vector of 


\section{R. DURRER}

the slicing are given by $\alpha=a(1+A)$ and $\boldsymbol{\beta}=-B^{i} \partial_{i}$. In first order,

$$
p_{0}=a p(1+A)-a p n_{i} B^{i},
$$

and in zeroth order, clearly,

$$
p_{i}=a p n_{i}
$$

Furthermore, the baryon four-velocity has the form $u^{0}=$ $a^{-1}(1-A), u^{i}=u^{0} v^{i}$ up to first order. This yields

$$
p^{\prime}=p_{\mu} u^{\mu}=p\left(1+n_{i}\left(v^{i}-B^{i}\right)\right) .
$$

Using this identity and performing the integration over photon energies, we find

$$
\begin{aligned}
\rho_{r} \frac{d \iota_{+}(n)}{d t^{\prime}} & =\rho_{r} \sigma_{T} n_{e}\left[1+4 n_{i}\left(v^{i}-B^{i}\right)\right. \\
& \left.+\frac{1}{4 \pi} \int \iota\left(n^{\prime}\right) \omega\left(n, n^{\prime}\right) d \Omega^{\prime}\right]
\end{aligned}
$$

The distribution of photons scattered out of the beam, has the well known form (see, e.g., Lifshitz and Pitajewski [1983])

$$
\frac{d f_{-}}{d t^{\prime}}=\sigma_{T} n_{e} f^{\prime}\left(p^{\prime}, \mathbf{n}\right)
$$

so that we finally obtain

$$
\begin{aligned}
C^{\prime} & =\frac{4 \pi}{\rho_{r} a^{4}} \int d p\left(\frac{d f_{+}}{d t^{\prime}}-\frac{d f_{-}}{d t^{\prime}}\right) p^{3} \\
& =\sigma_{T} n_{e}\left[\delta_{r}-\iota+4 n_{i}\left(v^{i}-B^{i}\right)\right. \\
& \left.+\frac{3}{16 \pi} n_{i j} \int \iota\left(n^{\prime}\right) n_{i j}^{\prime} d \Omega^{\prime}\right]
\end{aligned}
$$

where $\delta_{r}=(1 / 4 \pi) \int \iota(n) d \Omega$ is the photon energy density perturbation.

Using the definitions of the gauge-invariant variables $\mathcal{M}^{(S)}$ and $V^{(b)}$ for the photon brightness perturbation and the baryon velocity potential, we can write $C^{\prime}$ in gauge-invariant form

$C^{\prime}=4 \sigma_{T} n_{e}\left[\frac{1}{4} D_{g}^{(r)}-\mathcal{M}^{(S)}+n^{i} V_{i}^{(b)}+\frac{1}{2} n_{i j} M^{i j}\right]$,

with

$$
D_{g}^{(r)}=(1 / \pi) \int \mathcal{M}^{(S)} d \Omega
$$

and

$$
M^{i j} \equiv \frac{3}{8 \pi} \int \mathcal{M}^{(S)}\left(n^{\prime}\right) n_{i j}^{\prime} d \Omega^{\prime}
$$

Since the term in square brackets of (4.63) is already first order we can set $d t^{\prime}=d t$ which yields $C=\frac{d t^{\prime}}{d \eta} C^{\prime}=$ $\frac{d t}{d \eta} C^{\prime}=a C^{\prime}$. The Boltzmann equation for scalar perturbations expressed in terms of the gauge invariant variable $\mathcal{M}^{(S)}$ then becomes

$$
\begin{aligned}
\dot{\mathcal{M}}^{(S)} & +n^{i} \partial_{i} \mathcal{M}^{(S)}=n^{i} \partial_{i}(\Phi-\Psi)+a \sigma_{T} n_{e}\left[\frac{1}{4} D_{g}^{(r)}\right. \\
& \left.-\mathcal{M}^{(S)}-n^{i} \partial_{i} V^{(b)}+\frac{1}{2} n_{i j} M^{i j}\right]
\end{aligned}
$$

For vector and tensor perturbations we obtain in the same way

$$
\begin{aligned}
\dot{\mathcal{M}}^{(V)} & +i \mu k \mathcal{M}^{(V)}=-n^{i} n^{j} \sigma_{i \mid j} \\
& +a \sigma_{T} n_{e}\left[n^{i} V_{i}^{(V b)}+\frac{1}{2} n^{i j} M_{i j}^{(V)}-\mathcal{M}^{(V)}\right] \\
\dot{\mathcal{M}}^{(T)} & +i \mu k \mathcal{M}^{(T)}=-n^{i} n^{j} \dot{H}_{i j} \\
& +a \sigma_{T} n_{e}\left[n^{i j} M_{i j}^{(T)}-\mathcal{M}^{(T)}\right]
\end{aligned}
$$

\section{The tight coupling limit}

Before recombination, when $n_{e} \simeq \rho_{b} / m_{p}$,

$$
\begin{aligned}
\eta_{T} \equiv \frac{1}{a \sigma_{T} n_{e}} \sim & \frac{10}{\Omega_{b} h}(1+z)^{-\frac{3}{2}} \eta \ll \eta, \\
& z_{\mathrm{eq}} \gtrsim z \gtrsim z_{\mathrm{dec}}, \\
& \sim \frac{10}{\Omega_{b} h}\left(1+z_{\mathrm{eq}}\right)^{-\frac{1}{2}}(1+z)^{-1} \eta, \\
& z \gtrsim z_{\mathrm{eq}} .
\end{aligned}
$$

To lowest order in $\eta_{T}$, collisions force the photon distribution to be of the form

$$
\mathcal{M}^{(S)}=\frac{1}{4} D_{g}+n^{i} V_{i}^{(b)}+\frac{1}{2} n^{i j} M_{i j}
$$

the building up of higher moments is strongly suppressed by collisions.

During recombination, the number density of free electrons, $n_{e}$, decreases rapidly and the collision term becomes less and less important. Higher moments in the photon distribution develop by free streaming.

The collision term $C\left[\mathcal{M}^{(S)}\right]$ of equation (4.64) also appears in the equation of motion of the baryons as a drag. The Thomson drag force is given by

$$
F_{j}=\frac{\rho_{r}}{4 \pi} \int C\left[\mathcal{M}^{(S)}\right] n_{j} d \Omega=\frac{-4 a \sigma_{T} n_{e} \rho_{r}}{3}\left(M_{j}+V_{i}^{(b)}\right),
$$

with

$$
M_{j}=\frac{3 i}{4 \pi} \int n_{j} \mathcal{M}^{(S)} d \Omega .
$$

This yields the following scalar baryon equation of motion in an ionized plasma

$$
\dot{V}^{(b)}+(\dot{a} / a) V^{(b)}=k \Psi-\frac{4 a \sigma_{T} n_{e} \rho_{r}}{3 \rho_{b}}\left(-\hat{k}^{j} M_{j}+V^{(b)}\right),
$$

where we have added the drag force to the second eq. of (2.55) with $w=c_{s}^{2}=0$.

We now want to discuss equations $(4.64,4.71)$ in the limit of very many collisions. The comoving photon mean 
free path is given by $\eta_{T}=l_{T}=\left(a \sigma_{T} n_{e}\right)^{-1}$. In lowest order $\eta_{T} / \eta$ and $l_{T} / \lambda[13], \mathcal{M}^{(S)}$ is given by (4.69), and Eq. (4.71) implies

$$
-\hat{k}^{j} M_{j}+V^{(b)}=0 .
$$

Inserting the solution (4.69) in the Boltzmann equation (4.64) and integrating over directions this implies

$$
k V^{(b)}=k^{j} M_{j}=k V^{(r)}=\frac{-3}{4} \dot{D}_{g}^{(r)},
$$

Implying especially $V^{(b)}=V^{(r)} \equiv V$. Eq. (4.72) is equivalent to the energy conservation equation (2.55) for radiation. Using also (2.55) for baryons, $w=0$, we obtain

$$
\dot{D}_{g}^{(r)}=\frac{-4 k}{3} V^{(b)}=\frac{4}{3} \dot{D}_{g}^{(b)} .
$$

This shows that entropy per baryon is conserved, $\Gamma=0$. Before recombination, when the collisions are sufficiently frequent, baryons and photons are adiabatically coupled. Inserting (4.69) in (4.64) we find up to first order in $\eta_{T}$

$$
\begin{aligned}
\mathcal{M}^{(S)} & =D_{g}^{(r)}-4 i n^{j} k_{j} V+\frac{1}{2} n_{i j} M^{i j} \\
& -\eta_{T}\left[\dot{D}_{g}^{(r)}-4 i n^{j} k_{j} \dot{V}+\frac{1}{2} n_{i j} \dot{M}^{i j}\right. \\
& +i n^{j} k_{j} D_{g}^{(r)}+4 n^{i} n^{j} k_{i} k_{j} V \\
& \left.+\frac{i}{2} n^{i} n_{m j} k_{i} M^{m j}-i 4 n^{j} k_{j}(\Phi-\Psi)\right] .
\end{aligned}
$$

Using (4.73) to calculate the drag force yields

$$
F_{j}=i k_{j}\left(\rho_{r} / 3\right)\left[4 \dot{V}-D_{g}^{(r)}+4(\Phi-\Psi)\right] .
$$

Inserting $F_{j}$ in (4.71), we obtain

$$
\begin{aligned}
& \left(\rho_{b}+(4 / 3) \rho_{r}\right) \dot{V}+\rho_{b}(\dot{a} / a) V=\left(\rho_{r} / 3\right) D_{g}^{(r)} \\
& \quad+\left(\rho_{b}+(4 / 3) \rho_{r}\right) \Psi-\left(4 \rho_{r} / 3\right) \Phi .
\end{aligned}
$$

This is equivalent to momentum conservation, the second equation of (2.55) for $\rho=\rho_{b}+\rho_{r}, p=\rho_{r} / 3$ and $\Gamma=\Pi=0$, if we use

$$
D_{g}^{(r)}=(4 / 3) D_{g}^{(b)} \quad \text { and } \quad D_{g}=\frac{\rho_{r} D_{g}^{(r)}+\rho_{b} D_{g}^{(b)}}{\rho_{b}+\rho_{r}}
$$

In this limit therefore, baryons and photons behave like a single fluid with density $\rho=\rho_{r}+\rho_{b}$ and pressure $p=\rho_{r} / 3$.

From (2.55) we can derive a second order equation for $D_{g}$. This equation can be simplified if expressed in terms of the variable $D$ related by $(3.1)$. We obtain

$$
\begin{gathered}
\ddot{D}+c_{s}^{2} k^{2} D+\left(1+3 c_{s}^{2}-6 w\right)(\dot{a} / a) \dot{D}-3[w(\ddot{a} / a) \\
\left.-(\dot{a} / a)^{2}\left(3\left(c_{s}^{2}-w\right)-(1 / 2)(1+w)\right)\right] D=0 .
\end{gathered}
$$

For small wavelengths (sub-horizon), which are however sufficiently large for the fluid approximation to be valid, $1 / \eta_{T} \gg c_{s} k \gg 1 / \eta$, we may drop the term in square brackets. The ansatz $D(t)=A(t) \exp \left(-i \int k c_{s} d t\right)$ then eliminates the term of order $c_{s}^{2} k^{2}$. For the terms of order $c_{s} k / \eta$ we obtain the equation

$$
2 \dot{A} / A+\left(1+3 c_{s}^{2}-6 w\right)(\dot{a} / a)+\dot{c_{s}} / c_{s}=0 .
$$

For the case $c_{s}^{2}=w=$ const. This equation is solved by $A \propto(k \eta)^{1-\nu}$ with $\nu=2 /(3 w+1)$, i.e., the short wave limit. In our situation we have

$$
\begin{aligned}
& w=\frac{\rho_{r}}{3\left(\rho_{r}+\rho_{b}\right)}, \\
& c_{s}^{2}=\frac{\dot{\rho_{r}}}{3\left(\dot{\rho}_{r}+\dot{\rho}_{b}\right)}=\frac{(4 / 3) \rho_{r}}{4 \rho_{r}+3 \rho_{b}}, \\
& \dot{c}_{s} / c_{s}=-3 / 2(\dot{a} / a) \frac{\rho_{b}}{4 \rho_{r}+3 \rho_{b}} .
\end{aligned}
$$

Using all this, one finds that

$$
A=\left(\frac{\rho_{b}+(4 / 3) \rho_{r}}{c_{s}\left(\rho_{r}+\rho_{b}\right)^{2} a^{4}}\right)^{1 / 2}=\left(\frac{\rho+p}{c_{s} \rho^{2} a^{4}}\right)^{1 / 2}
$$

solves (4.74) exactly, so that we finally obtain the approximate solution for the tightly coupled matter radiation fluid on sub-horizon scales

$$
D(t) \propto\left(\frac{\rho+p}{c_{s} \rho^{2} a^{4}}\right)^{1 / 2} \exp \left(-i k \int c_{s} d \eta\right) .
$$

Note that this short wave approximation is only valid in the limit $\eta \gg 1 /\left(c_{s} k\right)$, thus the limit to the matter dominated regime, $c_{s} \rightarrow 0$ cannot be performed. In the limit to the radiation dominated regime, $c_{s}^{2} \rightarrow 1 / 3$ and $\rho \propto a^{-4}$ we recover the acoustic waves with constant amplitude which we have already found in the last subsection. But also in this limit, we still need matter to ensure $\eta_{T}=1 /\left(a \sigma_{T} n_{e}\right) \ll \eta$. In the opposite case, $\eta_{T} \gg \eta$, radiation does not behave like an ideal fluid but it becomes collisionless and has to be treated with Liouville's equation ((4.64) without the collision term).

\section{Damping by photon diffusion}

In this subsection we discuss the Boltzmann equation in the next order, $\left(\eta_{T} / \eta\right)^{2}$. In this order we will obtain the damping of fluctuations in an ionized plasma due to the finiteness of the mean free path; the non-perfect coupling. We follow the treatment by Peebles [21] (using our gauge-invariant approach instead of synchronous gauge). Again we consider Eqs. (4.64) and (4.71), but since we are mainly interested in collisions which take place on time scales $\eta_{T} \ll \eta$, we neglect gravitational effects and the time dependence of the coefficients. We can then look for solutions of the form

$$
V \propto \mathcal{M}^{(S)} \propto \exp (i(\mathbf{k} \cdot \mathbf{x}-\omega \eta))
$$

In (4.64) and (4.71) this yields (neglecting also the angular dependence of Compton scattering, i.e., the term $\left.n_{i j} M^{i j}\right)$ 


\section{R. DURRER}

$$
\mathcal{M}^{(S)}=\frac{1}{4} \frac{D_{g}^{(r)}-4 i \mathbf{k} \cdot \mathbf{n} V}{1-i \eta_{T}(\omega-\mathbf{k} \cdot \mathbf{n})}
$$

and

$$
\eta_{T} \mathbf{k} \omega V=\left(4 \rho_{r} / 3 \rho_{b}\right)(i \mathbf{k} V+\mathbf{M}),
$$

with $\mathbf{M}=(3 / 4 \pi) \int \mathbf{n} \mathcal{M}^{(S)} d \Omega$. Integrating (4.64) over angles, one obtains $\dot{D}_{g}^{(r)}+(i / 3) k_{j} M^{i}=0$. With our ansatz therefore $\mathbf{k} \cdot \mathbf{M}=3 \omega D_{g}^{(r)}$. Using this after scalar multiplication of (4.77) with $\mathbf{k}$, we find, setting $R=$ $3 \rho_{b} / 4 \rho_{r}$,

$$
V=\frac{(3 / 4) \omega D_{g}^{(r)}}{\eta_{T} k^{2} R \omega-i k^{2}} .
$$

Inserting this result for $V$ in (4.76) leads to

$$
\mathcal{M}^{(S)}=\frac{D_{g}^{(r)}}{4} \frac{1+\frac{3 \mu \omega / k}{1-i \eta_{T} \omega R}}{1-i \eta_{T}(\omega-k \mu)},
$$

where we have set $\mu=\hat{\mathbf{k}} \cdot \mathbf{n}$. This is the result of Peebles [21], where the same calculation is performed in synchronous gauge. Like there (§92), one obtains in lowest non-vanishing order $\omega \eta_{T}$ the following dispersion relation: Using

$$
\frac{1}{2} \int_{-1}^{1} \mathcal{M}^{(S)} d \mu=\frac{D_{g}^{(r)}}{4},
$$

which yields

$$
1=\frac{1}{2} \int_{-1}^{1} \frac{1+\frac{3 \mu \omega / k}{1-i \eta_{T} \omega R}}{1-i \eta_{T}(\omega-k \mu)} d \mu
$$

one finds

$$
\omega=\omega_{0}-i \gamma
$$

with

$$
\omega_{0}=k /[3(1+R)]^{1 / 2}
$$

and

$$
\gamma=\left(k^{2} \eta_{T} / 6\right) \frac{R^{2}+\frac{4}{5}(R+1)}{(R+1)^{2}} .
$$

In the baryon dominated regime, $R \geq 1$, therefore

$$
\gamma \approx k^{2} \eta_{T} / 6
$$

(If the angular dependence of Thompson scattering is not neglected, the term $\frac{4}{5}(R+1)$ in Eq. (IV C 3 ) becomes $\frac{8}{9}(R+1)$. If also polarization is taken into account, one obtains $\frac{16}{15}(R+1)$.)

Posing $k_{\text {damp }} \eta_{T} / 6=1$, this leads to a damping scale $\lambda_{\text {damp }} \sim \eta_{T}\left(\eta_{\text {dec }}\right) / 2$, which is projected in the microwave sky to an angle

$$
\vartheta_{\mathrm{damp}} \sim \frac{\eta_{T}\left(\eta_{\mathrm{dec}}\right)}{2 \chi\left(\eta_{0}-\eta_{\mathrm{dec}}\right)} .
$$

For $\kappa=0$ this corresponds to a few arc minutes and to the harmonic number

$$
\ell_{\mathrm{damp}}=\pi / \vartheta_{\mathrm{damp}} \simeq \frac{\pi \eta_{0}}{20 \eta_{T}\left(\eta_{\mathrm{dec}}\right)} \simeq \frac{\left(1+z_{\mathrm{dec}}\right)^{2}}{20} \Omega_{b} h .
$$

This estimate is very crude since we are using the approximation for $\eta_{T}$ from the tight coupling regime just where coupling stops to be tight, and we assume an arbitrary value of $n_{e} \sim 0.1 n_{b}$ at the moment of decoupling. Both these errors enhance the value of $\ell_{\text {damp }}$ somewhat. Numerical results give

$$
\ell_{\text {damp }} \sim 800-1000
$$

in a $\kappa=0$ universe. In open (closed) universes, this scale (which of course also depends on $\Omega_{b}$ ) is moved to larger (lower) $\ell$ due to projection. A reasonable approximation for the damping harmonic is

$$
\ell_{\text {damp }} \sim 1000\left(\frac{\Omega_{b} h}{0.02\left(1-\Omega_{\kappa}\right)^{1 / 2}}\right) .
$$

Temperature fluctuations on smaller scales, $\ell>\ell_{\text {damp }}$ are exponentially damped by photon diffusion.

\section{Polarization and moment expansion}

Thomson scattering is not isotropic. And what is more, for a non-isotropic photon distribution it depends on the polarization: Even if the incident photon beam is unpolarized, the scattered beam will be, unless the incident distribution is perfectly isotropic. In the previous section we have neglected this effect by summing over initial polarizations and averaging over final polarizations. Now we want to derive the difference in the Boltzmann equation taking into account polarization.

Polarization is usually characterized by means of the Stokes parameters [22-24].

For a harmonic electromagnetic wave with electric field

$$
\mathbf{E}(\mathbf{x}, t)=\left(\varepsilon_{1} E_{1}+\varepsilon_{2} E_{2}\right) e^{i \omega(\mathbf{n} \mathbf{x}-t)},
$$

where $\mathbf{n}, \varepsilon_{1}$ and $\varepsilon_{2}$ form an orthonormal basis and the complex field amplitudes are parameterized as $E_{j}=$ $a_{j} e^{i \delta_{j}}$, the Stokes parameters are given by

$$
\begin{aligned}
& I=a_{1}^{2}+a_{2}^{2}, \\
& Q=a_{1}^{2}-a_{2}^{2}, \\
& U=2 a_{1} a_{2} \cos \left(\delta_{2}-\delta_{1}\right), \\
& V=2 a_{1} a_{2} \sin \left(\delta_{2}-\delta_{1}\right) .
\end{aligned}
$$

$I$ is the intensity of the wave (whose perturbation $\imath$ has been introduced in the previous section), while $Q$ is a measure of the strength of linear polarization (the ratio of the principal axis of the polarization ellipse). $V$ measures circular polarization which is not generated by 
Thomson scattering and therefore $V$ vanishes if the initial circular polarization vanishes (which we assume). $U$ is then determined via the identity $I^{2}=Q^{2}+U^{2}$.

Since $Q$ vanishes in the background, to first order it obeys the unperturbed Boltzmann equation,

$$
\partial_{\eta} Q+i n^{j} k_{j} Q-\Gamma_{j k}^{(S) i} n^{j} n^{k} \frac{\partial Q}{\partial n^{i}}=C[Q],
$$

where $C$ is the collision integral. The same type of equation, with a somewhat different collision integral is satisfied by $U$. The collision integral for $V$ does not couple to $I, Q$ or $U$ and hence $V \equiv 0$ is a consistent solution.

An explicit derivation of the following Boltzmann hierarchy including polarization is presented in Appendix III A. Here we just repeat the necessary definitions and the results.

The brightness anisotropy $\mathcal{M}$ and the non-vanishing Stokes parameters $Q$ and $U$ can be expanded as

$$
\mathcal{M}(\eta, \mathbf{k}, \mathbf{n})=\sum_{\ell} \sum_{m=-2}^{2} \mathcal{M}_{\ell}^{(m)}(\eta, k)_{0} G_{\ell}^{m}(\mathbf{n})
$$

The $B$-mode vanishes for scalar perturbations, ${ }^{0} B_{l} \equiv 0$

$$
\begin{aligned}
Q(\eta, \mathbf{k}, \mathbf{n}) & \pm i U(\eta, \mathbf{k}, \mathbf{n})=\sum_{\ell} \sum_{m=-2}^{2}\left(E_{\ell}^{(m)}\right. \\
& \left. \pm i B_{\ell}^{(m)}\right)_{2} G_{\ell}^{m}(\mathbf{n})
\end{aligned}
$$

The special functions ${ }_{s} G_{\ell}^{m}$ are described in Appendix III A. The coefficients $m=0, m= \pm 1$ and $m= \pm 2$ describe the scalar $(S)$, vector $(V)$ and tensor $(T)$ components respectively. The Boltzmann equation for the coefficients $X_{\ell}^{(m)}$ is given by

$$
\begin{aligned}
& \dot{\mathcal{M}}_{\ell}^{(m)}-k\left[\frac{0 \kappa_{\ell}^{m}}{2 \ell-1} \mathcal{M}_{\ell-1}^{(m)}-\frac{0 \kappa_{\ell+1}^{m}}{2 \ell+3} \mathcal{M}_{\ell+1}^{(m)}\right]=-n_{e} \sigma_{T} a \mathcal{M}_{\ell}^{(m)}+S_{\ell}^{(m)}, \quad(\ell \geq m), \\
& \dot{E}_{\ell}^{(m)}-k\left[\frac{2 \kappa_{\ell}^{m}}{2 \ell-1} E_{\ell-1}^{(m)}-\frac{2 m}{\ell(\ell+1)} B_{\ell}^{(m)}-\frac{2 \kappa_{\ell+1}^{m}}{2 \ell+3} E_{\ell+1}^{(m)}\right]=-n_{e} \sigma_{T} a\left[E_{\ell}^{(m)}+\sqrt{6} C^{(m)} \delta_{\ell, 2},\right. \\
& \dot{B}_{\ell}^{(m)}-k\left[\frac{2 \kappa_{\ell}^{m}}{2 \ell-1} B_{\ell-1}^{(m)}+\frac{2 m}{\ell(\ell+1)} E_{\ell}^{(m)}-\frac{2 \kappa_{\ell+1}^{m}}{2 \ell+3} B_{\ell+1}^{(m)}\right]=-n_{e} \sigma_{T} a B_{\ell}^{(m)} .
\end{aligned}
$$

where we set

$$
\begin{aligned}
& S_{0}^{(0)}=n_{e} \sigma_{T} a \mathcal{M}_{0}^{(0)}, \quad S_{1}^{(0)}=n_{e} \sigma_{T} a 4 V_{b}+4 k(\Psi-\Phi), \quad S_{2}^{(0)}=n_{e} \sigma_{T} a C^{(0)} \\
& S_{1}^{(1)}=n_{e} \sigma_{T} a 4 \omega_{b}, \quad S_{2}^{(1)}=n_{e} \sigma_{T} a C^{(1)}+4 \Sigma, \quad S_{2}^{(2)}=n_{e} \sigma_{T} a C^{(2)}+4 \dot{H}
\end{aligned}
$$

and $C^{(m)}=\frac{1}{10}\left[\mathcal{M}_{2}^{(m)}-\sqrt{6} E_{2}^{(m)}\right]$. The coupling coefficients are

$$
{ }_{s} \kappa_{\ell}^{m}=\sqrt{\frac{\left(\ell^{2}-m^{2}\right)\left(\ell^{2}-s^{2}\right)}{\ell^{2}}} .
$$

The CMB temperature and polarization power spectra are given in terms of the expansion coefficients $\mathcal{M}_{\ell}^{(m)}$, $E_{\ell}^{(m)}$ and $B_{\ell}^{(m)}$ as

$$
(2 \ell+1)^{2} C_{\ell}^{X Y(m)}=\frac{n_{m}}{8 \pi} \int k^{2} d k X_{\ell}^{(m)} Y_{\ell}^{(m) *},
$$

where $n_{m}=1$ for $m=0$ and $n_{m}=2$ for $m=1,2$, accounting for the number of modes. Since $B$ is parity odd, the only non-vanishing cross correlation spectrum is $C^{\mathrm{TE}}$.

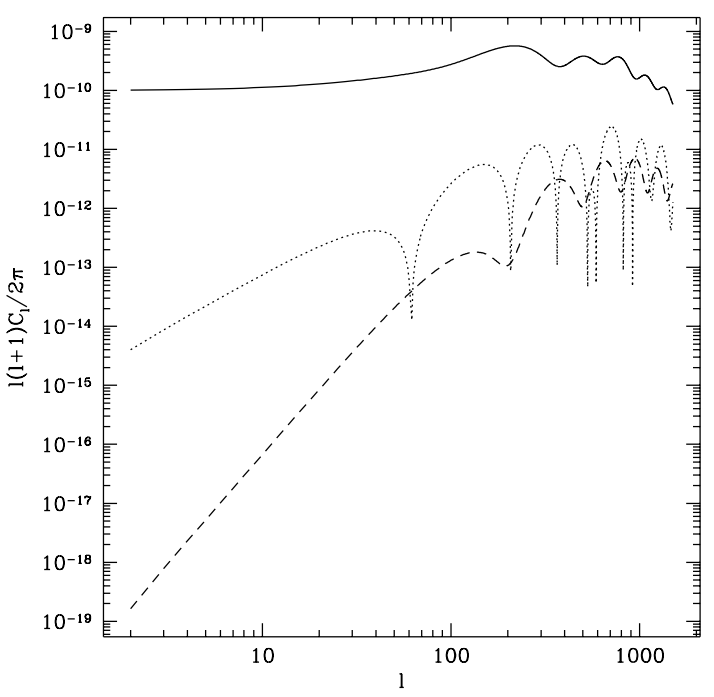

Fig. 7. The temperature anisotropy (solid), the polarization (dashed) and their correlation (dotted) are shown for a purely scalar standard CMD model. 
The Boltzmann hierarchy presented above can be solved numerically with publicly available fast numerical codes like CMBfast [37] or CAMCODE [38]. This enables us to compute the CMB anisotropy and polarization spectra for many different values of cosmological parameters, and compare them with present data.

\section{E. Parameter estimation}

In the last paragraph of this section of this section we make some general remarks about the dependence of the CMB anisotropy spectrum on different parameters and about degeneracies. We start by enumerating the relevant physical processes.

\section{Physical processes}

- Before recombination, photons and baryons form a tightly coupled fluid which performs acoustic oscillations on sub-horizon scales.

- Depending on the initial conditions, these oscillations are sine waves (isocurvature case) or cosine waves (adiabatic case).

- After recombination, the photons move along perturbed geodesics, only influenced by the metric perturbations.

- Vector perturbations of the metric decay as $a^{-2}$ after creation and their effects on CMB anisotropies are negligible for models where initial fluctuations are created early, e.g., during an inflationary phase. This is different for models which constantly seed fluctuations in the geometry, e.g., topological defects.

- Tensor perturbations of the metric have constant amplitude on super-horizon scales and perform damped oscillations $\propto a^{-1}$ once they enter the horizon.

- Scalar perturbations of the metric are roughly constant if they enter the horizon only after the time of matter and radiation equality. On scales which enter the horizon before equality they are damped by a factor $\left(z_{\text {eq }} / z_{\text {in }}\right)^{2}$, where $z_{\text {eq }}$ and $z_{\text {in }}$ are the redshift of equality and of horizon crossing, respectively.

- Perturbations on small scales, $k \gtrsim k_{T} \simeq$ $\left(\Omega_{b} h / 20\right)\left(z_{\mathrm{dec}}+1\right)^{2} H_{0}$ are exponentially damped by collisional damping during recombination (Silk damping).

\section{Scale dependence}

- On large scales (larger than the horizon scale at recombination, $\ell \lesssim \ell_{H} \simeq \pi / \vartheta_{H}$, with $\vartheta_{H}=$ $\eta_{\text {dec }} / \chi\left(\eta_{0}-\eta_{\text {dec }}\right)$, perturbations are dominated by gravitational effects: Inflationary models typically lead to $k^{3}\left\langle|\Psi-\Phi|^{2}\left(k, \eta_{\text {dec }}\right)\right\rangle \simeq$ const and $k^{3}\left\langle H^{2}\right\rangle \simeq$ const on these scales. This implies a flat "Harrison-Zel'dovich" spectrum,

$$
\left(\frac{\Delta T}{T}\right)^{2}\left(\vartheta_{\ell}\right) \simeq \ell(\ell+1) C_{\ell} \simeq \text { const }, \quad \vartheta_{\ell}=\frac{\pi}{\ell} .
$$

- On intermediate scales, $\ell_{H}<\ell<\ell_{\text {damp }} \sim 800$, CMB anisotropies mainly reflect the acoustic oscillations of the photon/baryon plasma prior to recombination. The position of the first peak is severely affected by initial conditions (adiabatic or isocurvature). For $\kappa=0$, the first contraction peak is at about $\ell_{1}^{(a)} \sim 200$ if the initial conditions are adiabatic, while the first contraction peak is at $\ell_{1}^{(i)} \sim 350$ for isocurvature initial conditions. The amplitude of and the distance between the peaks depend strongly on cosmological parameters.

- On small scales, $\ell_{\text {damp }}<\ell$, fluctuations are collisionally damped during recombination ("Silk damping"). The damping scale depends mainly on $\Omega_{b} h$ and $\Omega$.

\section{The main influence of cosmological parameters}

- Curvature, $h^{2} \Omega_{\kappa}$ :

- Mainly affects the inter-peak distance, $\Delta \ell$, and, for given initial conditions, the position of the first peak. Positive curvature lowers $\Delta \ell$ while negative curvature enhances it.

- Curvature also leads to an integrated SachsWolfe contribution which is especially important for $\kappa>0$ at very low $\ell$. Overall, this leads to some enhancement of the Sachs-Wolfe contribution and therefore (after normalization to the COBE measurements) to somewhat lower acoustic peaks.

- Baryon density, $\rho_{b}=\Omega_{b} h^{2} \cdot 10^{-29} \mathrm{~g} / \mathrm{cm}^{3}$ :

- A high baryonic density enhances the compression peaks and decreases the expansion peaks via the self-gravity of the baryons.

- It also reduces the damping scale, $\lambda_{T}=$ $1 /\left(a_{\mathrm{dec}} \sigma_{T} n_{\ell}\left(\eta_{\mathrm{dec}}\right)\right)$, leading to an increase in $\ell_{\text {damp }}$.

- Baryons decrease the plasma sound velocity, $c_{s}=1 / 3\left(1+\dot{\rho}_{b} / \dot{\rho}_{\gamma}\right)^{-1}$, and hence prolongs the oscillation period. This increases the spacing between acoustic peaks. 
- Cosmological Constant, $\Lambda=\frac{\Omega_{\Lambda} h^{2}}{8 \pi G} 10^{-29} \mathrm{~g} / \mathrm{cm}^{3}$ :

The presence of a cosmological constant at fixed $\Omega_{\text {tot }}=\Omega_{m}+\Omega_{\Lambda}$ delays the epoch of equal matter and radiation. During the radiation dominated era, the gravitational potential is not constant, but decays as soon as a given scale enters the horizon. If $\eta_{\text {eq }} \sim \eta_{\text {dec }}$ this induces an integrated Sachs-Wolfe (ISW) contribution which boosts mainly the first acoustic peak. $\Omega_{\Lambda}$ also boosts the late integrated Sachs-Wolfe contribution.

- Hubble Parameter, $H_{0}=100 \mathrm{~h} \mathrm{~km} /(\mathrm{s} \cdot \mathrm{Mpc})$ : The influence of the Hubble parameter is complicated and depends sensitively on the variables which are kept fixed during its variation $\left(\Omega_{0}\right.$ or $\omega_{\bullet}=h^{2} \Omega_{\text {。 }}$. As one example of its influence: for fixed curvature and cosmological constant, lowering the Hubble parameter also delays the epoch of equal matter and radiation, $\eta_{\text {eq }} \rightarrow \eta_{\text {dec }}$, since

$$
z_{\mathrm{eq}}+1=\frac{\Omega_{m}}{\Omega_{\mathrm{rad}}} \simeq 2.4 \cdot 10^{4} \Omega_{m} h^{2}
$$

Therefore the same type of ISW contribution as for $\Lambda$-models boosts the first acoustic peak.

\section{- Initial conditions:}



- A tensor contribution enhances the large scales fluctuations but not the acoustic peaks, thereby lowering their relative amplitude.

- A "blue" fluctuation spectrum, $n>1$, enhances fluctuations on smaller scales and raises thereby the acoustic peaks.

\section{Degeneracy}

One important issue in determining cosmological parameters from CMB anisotropy measurements is the choise of good variables, which requires physical insight in how anisotropies are influenced. As we have argued before, the Hubble parameter, $h$ is not a good variable since its influence is very complicated. It enters the cosmic densities $\rho_{\bullet} \propto \Omega_{\bullet} h^{2}$ and the length scales like $\eta_{e q}$ or $\eta_{\text {dec }}$. Another limitation for parameter estimation from CMB anisotropies is degeneracy. We illustrate here just one example. As we have discussed in Section 3, the position of the first acoustic peak only depends on the sound horizon, $\tau_{s}=\int^{\eta_{\mathrm{dec}}} c_{s} d \eta$ and the angular diameter distance to the last scattering surface, $\chi\left(\eta_{0}-\eta_{\text {dec }}\right)$. The distance between subsequent peaks in the CMB power spectrum is proportional to

$$
\Delta \ell=\frac{\chi\left(\eta_{0}-\eta_{\mathrm{dec}}\right)}{\tau_{s}}
$$

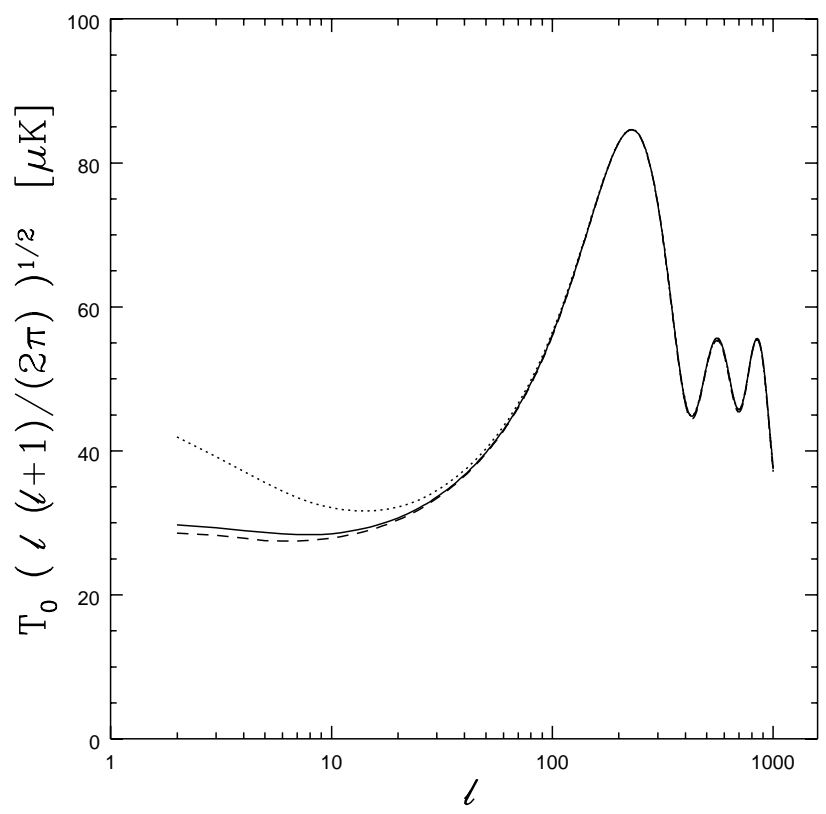

Fig. 8. Left: The lines of constant $R$ are shown in the $\Omega_{\Lambda}-\Omega_{m}$ plane. The values $\Omega_{\Lambda}, \Omega_{m}$ for which the CMB anisotropy spectra are shown right are indicated as black dots. Right: Three CMB anisotropy spectra with different values of $\Omega_{\Lambda}$ and $\Omega_{m}$ but fixed $R$ are shown. For $\ell \gtrsim 50$ these spectra are clearly degenerate. The solid line represents a flat model, while the dotted line corresponds to a closed and the dashed line to an open universe. 


\section{R. DURRER}

In Fig. 8 (left panel) we show lines of constant $R=$ $\Delta \ell / \Delta \ell_{0}$ in the $\Omega_{m}-\Omega_{\Lambda}$ plane. Here $\Delta \ell_{0}=\Delta \ell_{0}\left(\Omega_{\Lambda}=\right.$ $\Omega_{k}=0$ ) is the value of $\Delta \ell$ in a spatially flat universe with vanishing cosmological constant. To the right the CMB anisotropy spectra for scalar perturbations with fixed index $n=1$ and fixed values of the matter density $\omega_{m}$ and the baryon density $\omega_{b}$. But the cosmological constant and $h$ vary, so that $\Omega_{\Lambda}$ and $\Omega_{m}$ correspond to the values indicated by bullets on the left panel. Clearly, for $\ell>50$ these spectra are perfectly degenerate. On the other hand, due to cosmic variance, the low $\ell$ CMB anisotropies will never be known to very good accuracy so that this degeneracy cannot be lifted by CMB anisotropy observations alone. Additional data like the supernova type Ia measurements, observations of the galaxy distribution (large scale structure) or CMB polarization are needed.

There are also other degeneracies like the optical depth to reionization and the tensor contribution or the scalar spectral index and the tensor contribution. The important lesson to learn is that even if the very stringent model assumptions are correct, we still need other data to measure cosmological parameters and especially we will only feel comfortable with a sufficient amount of redundancy.

\section{OBSERVATIONS AND RESULTS}

In this short, final section we want to discuss briefly the experimental situation which is very much in flow and may have changed considerably already at the moment when this review appears. It has been clear for a long time that, if initial fluctuations have led to the formation of large scale structure by gravitational instability, they should have induced fluctuations in the cosmic microwave background [27,28]. Before spring 1992, however, only the dipole anisotropy had been detected $[16,17]$. Its value is $[7]$

$$
\left\langle\left(\frac{\Delta T}{T}\right)^{2}\right\rangle^{\text {dipole }}=(1.528 \pm 0.004) \times 10^{-6}
$$

After many upper limits, the DMR experiment aboard the COBE satellite measured for the first time convincingly positive anisotropies [8]. It found

$$
\left\langle(\Delta T)^{2}\right\rangle(\theta) \sim(30 \mu \mathrm{K})^{2}
$$

on all angular scales $\theta \geq 7^{\circ}$. Many more positive measurements have been performed since then. In Fig. 9 we just show the COBE DMR results [39] together with the three most recent experiments, BOOMERANG [40], MAXIMA-1 [41] and DASI [42]


Fig. 9. The measured temperature anisotropies, $\ell(\ell+1) C_{\ell}$ are shown in a lin-lin plot (left) and in a log-lin plot (right) with the theoretical curve from a standard, adiabatic cold dark matter model. The data points shown are those from COBE DMR (solid, low $\ell$ ), BOOMERANG (solid), DASI (dashed) and MAXIMA-1 (dotted). 
As one sees in this figure, present data agrees very well with a simple flat model of purely scalar, scale invariant, $n_{s}=1$, adiabatic fluctuations with cosmological parameter $\omega_{b}=0.02, \Omega_{\kappa}=0, \Omega_{\Lambda}=0.7, h=0.65$ which are also preferred from other cosmological data. However, the error-bars are still considerable.

The experiments can be split into three classes: Satellite experiments, balloon-borne experiments and ground based experiments. The technical and economical advantages of ground based experiments are obvious. Their main problem is atmospheric fluctuation. This can be reduced by two methods:

- Choose a very high altitude and very cold site, e.g., the south pole. Several experiments like SP, Python and White Dish have chosen this site.

- Measure anisotropies on small scales, preferably by interferometry (DASI, CAT, VSA, Jodrell Bank).

Balloon-borne experiments flying at about $40 \mathrm{~km}$ altitude have less problems with the Earth atmosphere but they face the following difficulties:

- They are limited in weight.

- They cannot be manipulated at will in flight.

- They have a rather short duration.

- To secure all the data taken on the balloon, they have to be recovered intact.

Yet the advantages of overcoming the atmosphere are so significant that many groups have chosen this approach, like, e.g., MAXIMA-1, TopHat, etc. The BOOMERANG experiment combines the two advantages of a cold site and balloon altitude. It has performed a long-duration flight (10 days) on the south pole in December 1998.

The third possibility are satellite experiments. They avoid atmospheric problems altogether, but this solution is very expensive. So far two satellite experiments have been launched: COBE in 1989 (NASA mission) and MAP in June 2001 (Microwave Anisotropy Probe, a NASA MIDEX mission), one more is planned: PLANCK, an ESA medium size mission of the "Horizon 2000" program, to be launched in 2007.

As I am writing this lines, MAP has safely arrived at its destination, the Lagrange point L2 of the sun-earth system. It will perform measurements at five frequencies in the range from 22 to $90 \mathrm{GHz}$. The instruments of PLANCK will operate at nine frequencies, covering 20 to $800 \mathrm{GHz}$. At low frequencies (below $100 \mathrm{GHz}$ ) radio receivers are used (so called "HEMTs", high electron mobility transistors) while the high frequency instruments are bolometers. Recent progress in detector technologies should enable the two new satellites to perform significantly better than COBE - the radio receivers of PLANCK, e.g., are supposed to be 1000 times more sensitive than the ones used for COBE, and the angular resolution has improved from seven degrees to four arc minutes. For more details also on other experiments see:

\section{- http://astro.estec.esa.nl/PLAIICK \\ - http://map.gsfc.nasa.gov \\ - http://н日н.gsfc.nasa.gov/astro/cobe/cobehome.html

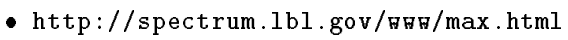 \\ - http://oberon.roma1.infn.it/boomerang/}

I finish this short section with Table 1 which shows the ranges for the cosmological parameters $\Omega_{\text {tot }}=1-$ $\Omega_{\kappa}, h^{2} \Omega_{b}$ and $n_{s}$ as determined purely by CMB anisotropies. Except for the last reference, a purely scalar spectrum of adiabatic fluctuations is assumed. The parameter estimation process also assumes 'weak priors' on the values of other cosmological observables, like, e.g., that the age of the Universe be larger than 10 Gyrs. or $0.4<h<0.9$. I do not comment this table much further but refer the reader to the original literature and many improved papers on this subject which will appear shortly.

\begin{tabular}{|c|c|c|c|c|c|}
\hline Ref. & Data & $\Omega_{\text {tot }}$ & $\Omega_{b} h^{2}$ & $n_{s}$ & errors \\
\hline$[43]$ & BOOM and DMR data & $1.02_{-0.05}^{+0.06}$ & $0.022_{-0.003}^{+0.004}$ & $0.96_{-0.09}^{+0.1}$ & $1-\sigma$ errors \\
{$[44]$} & DASI and DMR data & $1.05_{-0.06}^{+0.06}$ & $0.022_{-0.004}^{+0.004}$ & $1.01_{-0.07}^{+0.09}$ & $1-\sigma$ errors \\
{$[45]$} & MAX and DMR data & $0.90_{-0.16}^{+0.18}$ & $0.0325_{-0.0125}^{+0.0125}$ & $0.99_{-0.14}^{+0.14}$ & $2-\sigma$ errors \\
& $\begin{array}{c}\text { all data, no priors } \\
\text { allows also tensor mode }\end{array}$ & $1.06_{-0.13}^{+0.59}$ & $0.02_{-0.01}^{+0.06}$ & $0.93_{-0.16}^{+0.75}$ & $2-\sigma$ errors \\
\hline
\end{tabular}

Table 1. Some results from parameter estimations from recent CMB data alone. The errors given are formal 1 or $2-\sigma$ errors which assume the underlying model to be correct and no systematic problems in the data. They are obtained by marginalization or maximization over all other model parameters. 


\section{R. DURRER}

Clearly, the results shown in Table 1 are very consistent. It is interesting to note, how the upper limit on the scalar spectral index deteriorates if one allows for a tensor component. This is one of the degeneracies in the CMB data which can be broken by including large scale structure data in the analysis (see [46]). Other cosmological parameters are not well constrained by CMB data alone. However, if CMB data is combined with SN1a and large scale structure data, the error bars are significantly reduced and evidence for a non-vanishing cosmological constant $\Omega_{\Lambda} \sim 0.7$ becomes very strong (see $[43,44,46]$ ).

\section{ACKNOWLEDGMENT}

It is a pleasure to thank Pedro Ferreira, Roman Juszkiewicz, Martin Kunz, Alessandro Melchiorri, Bo- hdan Novosyadlyj and Norbert Straumann for stimulating discussions. I am also very grateful to Martin Kunz for his help with the preparation of this manuscript. Finally, thanks for hospitality go to the Institute for Advanced Study in Princeton, where this review was terminated.

\section{APPENDIX A: THE $C_{\ell}$ 'S FROM GRAVITATIONAL WAVES}

We consider metric perturbations which are produced by some isotropic random process (for example during inflation). After production, they evolve according to a deterministic equation of motion. By reasons of isotropy and due to symmetry, the correlation functions of $h_{i j}(\mathbf{k}, \eta)$ have to be of the form

$$
\begin{aligned}
& \left\langle h_{i j}(\mathbf{k}, \eta) h_{l m}^{*}\left(\mathbf{k}, \eta^{\prime}\right)\right\rangle=\left[k_{i} k_{j} k_{l} k_{m} H_{1}\left(k, \eta, \eta^{\prime}\right)+\left(k_{i} k_{l} \delta_{j m}+k_{i} k_{m} \delta_{j l}+k_{j} k_{l} \delta_{i m}+k_{j} k_{m} \delta_{i l}\right) H_{2}\left(k, \eta, \eta^{\prime}\right)\right. \\
& \left.+k_{i} k_{j} \delta_{l m} H_{3}\left(k, \eta, \eta^{\prime}\right)+k_{l} k_{m} \delta_{i j} H_{3}^{*}\left(k, \eta^{\prime}, \eta\right)+\delta_{i j} \delta_{l m} H_{4}\left(k, \eta, \eta^{\prime}\right)+\left(\delta_{i l} \delta_{j m}+\delta_{i m} \delta_{j l}\right) H_{5}\left(k, \eta, \eta^{\prime}\right)\right] .
\end{aligned}
$$

Here the functions $H_{a}$ are functions of the modulus $k=|\mathbf{k}|$ only. Furthermore, all of them except $H_{3}$ are hermitian in $\eta$ and $\eta^{\prime}$. This is the most general ansatz for an isotropic correlation tensor satisfying the required symmetries. To project out the tensorial part of this correlation tensor we act on $h_{i j}$ it with the tensor projection operator,

$$
T_{i j}^{m n}=P_{i}^{m} P_{j}^{n}-(1 / 2) P_{i j} P^{m n} \quad \text { with } \quad P_{i j}=\delta_{i j}-\hat{k}_{i} \hat{k}_{j} .
$$

This yields

$$
\begin{aligned}
\left\langle h_{i j}^{(T)}(\mathbf{k}, \eta) h_{l m}^{(T) *}\left(\mathbf{k}, \eta^{\prime}\right)\right\rangle & =H_{5}\left(k, \eta, \eta^{\prime}\right)\left[\delta_{i l} \delta_{j m}+\delta_{i m} \delta_{j l}-\delta_{i j} \delta_{l m}+k^{-2}\left(\delta_{i j} k_{l} k_{m}\right.\right. \\
& \left.\left.+\delta_{l m} k_{i} k_{j}-\delta_{i l} k_{j} k_{m}-\delta_{i m} k_{l} k_{j}-\delta_{j l} k_{i} k_{m}-\delta_{j m} k_{l} k_{i}\right)+k^{-4} k_{i} k_{j} k_{l} k_{m}\right] .
\end{aligned}
$$

From Eq. (4.17), we then obtain

$$
\begin{aligned}
\left\langle\frac{\Delta T}{T}(\mathbf{n}) \frac{\Delta T}{T}\left(\mathbf{n}^{\prime}\right)\right\rangle & \equiv \frac{1}{V} \int d^{3} x\left(\frac{\Delta T}{T}(\mathbf{n}, \mathbf{x}) \frac{\Delta T}{T}\left(\mathbf{n}^{\prime}, \mathbf{x}\right)\right) \\
& =\left(\frac{1}{2 \pi}\right)^{3} \int k^{2} d k d \Omega_{\hat{\mathbf{k}}} \int_{\eta_{d e c}}^{\eta_{0}} d \eta \int_{\eta_{d e c}}^{\eta_{0}} d \eta^{\prime} \exp \left(i \mathbf{k} \cdot \mathbf{n}\left(\eta_{0}-\eta\right)\right) \exp \left(-i \mathbf{k} \cdot \mathbf{n}\left(\eta_{0}-\eta^{\prime}\right)\right) \\
& \times\left[\left\langle\dot{h}_{i j}^{(T)}(\eta, \mathbf{k}) \dot{h}_{l m}^{(T) *}\left(\eta^{\prime}, \mathbf{k}\right)\right\rangle n_{i} n_{j} n_{l}^{\prime} n_{m}^{\prime}\right] .
\end{aligned}
$$

Here $d \Omega_{\hat{\mathbf{k}}}$ denotes the integral over directions in $\mathbf{k}$ space. We use the normalization of the Fourier transform

$$
\hat{f}(\mathbf{k})=\frac{1}{\sqrt{V}} \int d^{3} x \exp (i \mathbf{x} \cdot \mathbf{k}) f(\mathbf{x}), \quad f(\mathbf{x})=\frac{1}{(2 \pi)^{3}} \int d^{3} k \exp (-i \mathbf{x} \cdot \mathbf{k}) \hat{f}(\mathbf{k}),
$$

where $V$ is an (arbitrary) normalization volume.

We now introduce the form (A.3) of $\left\langle h^{(T)} h^{(T)}\right\rangle$. We further make use of the assumption that the perturbations have been created at some early epoch, e.g., during an inflationary phase, after which they evolved deterministically. The function $H_{5}\left(k, \eta, \eta^{\prime}\right)$ is thus a product of the form

$$
H_{5}\left(k, \eta, \eta^{\prime}\right)=H(k, \eta) \cdot H^{*}\left(k, \eta^{\prime}\right) .
$$

Introducing this in Eq. (A.4) yields 


$$
\begin{aligned}
\left\langle\frac{\Delta T}{T}(\mathbf{n}) \frac{\Delta T}{T}\left(\mathbf{n}^{\prime}\right)\right\rangle & =\left(\frac{1}{2 \pi}\right)^{3} \int k^{2} d k d \Omega_{\hat{\mathbf{k}}}\left[\left(\mathbf{n} \cdot \mathbf{n}^{\prime}\right)^{2}-1+\mu^{\prime 2}+\mu^{2}-4 \mu \mu^{\prime}\left(\mathbf{n} \cdot \mathbf{n}^{\prime}\right)+\mu^{2} \mu^{\prime 2}\right] \\
& \times \int_{\eta_{\mathrm{dec}}}^{\eta_{\mathrm{o}}} d \eta \int_{\eta_{\mathrm{d} e c}}^{\eta_{0}} d \eta^{\prime}\left[\dot{H}(k, \eta) \dot{H}^{*}\left(k, \eta^{\prime}\right) \exp \left(i k \mu\left(\eta_{0}-\eta\right)\right) \exp \left(-i k \mu^{\prime}\left(\eta_{0}-\eta^{\prime}\right)\right)\right],
\end{aligned}
$$

where $\mu=(n \cdot \hat{\mathbf{k}})$ and $\mu^{\prime}=\left(n^{\prime} \cdot \hat{\mathbf{k}}\right)$. To proceed, we use the identity [47]

$$
\exp \left(\left(i k \mu\left(\eta_{0}-\eta\right)\right)=\sum_{r=0}^{\infty}(2 r+1) i^{r} j_{r}\left(k\left(\eta_{0}-\eta\right)\right) P_{r}(\mu) .\right.
$$

Here $j_{r}$ denotes the spherical Bessel function of order $r$ and $P_{r}$ is the Legendre polynomial of degree $r$.

Furthermore, we replace each factor of $\mu$ in Eq. (A.6) by a derivative of the exponential $\exp \left(i k \mu\left(\eta_{0}-\eta\right)\right)$ with respect to $k\left(\eta_{0}-\eta\right)$ and correspondingly with $\mu^{\prime}$. We then obtain

$$
\begin{aligned}
\left\langle\frac{\Delta T}{T}(\mathbf{n}) \frac{\Delta T}{T}\left(\mathbf{n}^{\prime}\right)\right\rangle & =\left(\frac{1}{2 \pi}\right)^{3} \sum_{r, r^{\prime}}(2 r+1)\left(2 r^{\prime}+1\right) i^{\left(r-r^{\prime}\right)} \int k^{2} d k d \Omega_{\hat{\mathbf{k}}} P_{r}(\mu) P_{r^{\prime}}\left(\mu^{\prime}\right) \\
& \times\left[2\left(\mathbf{n} \cdot \mathbf{n}^{\prime}\right)^{2} \int d \eta d \eta^{\prime} j_{r}\left(k\left(\eta_{0}-\eta\right)\right) j_{r^{\prime}}\left(k\left(\eta_{0}-\eta^{\prime}\right)\right) \dot{H}(k, \eta) \dot{H}^{*}\left(k, \eta^{\prime}\right)\right. \\
& -\int d \eta d \eta^{\prime}\left[j_{r}\left(k\left(\eta_{0}-\eta\right)\right) j_{r^{\prime}}\left(k\left(\eta_{0}-\eta^{\prime}\right)\right)+j_{r}^{\prime \prime}\left(k\left(\eta_{0}-\eta\right)\right) j_{r^{\prime}}\left(k\left(\eta_{0}-\eta^{\prime}\right)\right)\right. \\
& \left.+j_{r}\left(k\left(\eta_{0}-\eta\right)\right) j_{r^{\prime}}^{\prime \prime}\left(k\left(\eta_{0}-\eta^{\prime}\right)\right)-j_{r}^{\prime \prime}\left(k\left(\eta_{0}-\eta\right)\right) j_{r^{\prime}}^{\prime \prime}\left(k\left(\eta_{0}-\eta^{\prime}\right)\right)\right] \dot{H}(k, \eta) \dot{H}^{*}\left(k, \eta^{\prime}\right) \\
& \left.-4\left(\mathbf{n} \cdot \mathbf{n}^{\prime}\right) \int d \eta d \eta^{\prime} j_{r}^{\prime}\left(k\left(\eta_{0}-\eta\right)\right) j_{r^{\prime}}^{\prime}\left(k\left(\eta_{0}-\eta^{\prime}\right)\right) \dot{H}(k, \eta) \dot{H}^{*}\left(k, \eta^{\prime}\right)\right] .
\end{aligned}
$$

Here only the Legendre polynomials, $P_{r}(\mu)$ and $P_{r^{\prime}}\left(\mu^{\prime}\right)$ depend on the direction $\hat{\mathbf{k}}$. To perform the integration $d \Omega_{\hat{\mathbf{k}}}$, we use the addition theorem for the spherical harmonics $Y_{r s}$,

$$
P_{r}(\mu)=\frac{4 \pi}{(2 r+1)} \sum_{s=-r}^{r} Y_{r s}(\mathbf{n}) Y_{r s}^{*}(\hat{\mathbf{k}})
$$

The orthogonality of the spherical harmonics then yields

$$
(2 r+1)\left(2 r^{\prime}+1\right) \int d \Omega_{\hat{\mathbf{k}}} P_{r}(\mu) P_{r^{\prime}}\left(\mu^{\prime}\right)=16 \pi^{2} \delta_{r r^{\prime}} \sum_{s=-r}^{r} Y_{r s}(\mathbf{n}) Y_{r s}^{*}\left(\mathbf{n}^{\prime}\right)=4 \pi \delta_{r r^{\prime}} P_{r}\left(\mathbf{n} \cdot \mathbf{n}^{\prime}\right)
$$

In Eq. (A.8) the integration over $d \Omega_{\hat{\mathbf{k}}}$ then leads to terms of the form $\left(\mathbf{n} \cdot \mathbf{n}^{\prime}\right) P_{r}\left(\mathbf{n} \cdot \mathbf{n}^{\prime}\right)$ and $\left(\mathbf{n} \cdot \mathbf{n}^{\prime}\right)^{2} P_{r}\left(\mathbf{n} \cdot \mathbf{n}^{\prime}\right)$. To reduce them, we use

$$
x P_{r}(x)=\frac{r+1}{2 r+1} P_{r+1}+\frac{r}{2 r+1} P_{r-1}
$$

Applying this and its iteration for $x^{2} P_{r}(x)$, we obtain

$$
\begin{aligned}
\left\langle\frac{\Delta T}{T}(\mathbf{n}) \frac{\Delta T^{*}}{T}\left(\mathbf{n}^{\prime}\right)\right\rangle= & \frac{1}{2 \pi^{2}} \sum_{r}(2 r+1) \int k^{2} d k \int d \eta d \eta^{\prime} \dot{H}(k, \eta) \dot{H}^{*}\left(k, \eta^{\prime}\right) \\
& \times\left\{\left[\frac{2(r+1)(r+2)}{(2 r+1)(2 r+3)} P_{r+2}+\frac{1}{(2 r-1)(2 r+3)} P_{r}+\frac{2 r(r-1)}{(2 r-1)(2 r+1)} P_{r-2}\right]\right. \\
& \times j_{r}\left(k\left(\eta_{0}-\eta\right)\right) j_{r}\left(k\left(\eta_{0}-\eta^{\prime}\right)\right)-P_{r}\left[j _ { r } \left(k\left(\eta_{0}-\eta\right) j_{r}^{\prime \prime}\left(k\left(\eta_{0}-\eta^{\prime}\right)\right)\right.\right. \\
& \left.+j_{r}\left(k\left(\eta_{0}-\eta^{\prime}\right)\right) j_{r}^{\prime \prime}\left(k\left(\eta_{0}-\eta\right)\right)-j_{r}^{\prime \prime}\left(k\left(\eta_{0}-\eta\right)\right) j_{r^{\prime}}^{\prime \prime}\left(k\left(\eta_{0}-\eta^{\prime}\right)\right)\right] \\
& \left.-4\left[\frac{r+1}{2 r+1} P_{r+1}+\frac{r}{2 r+1} P_{r-1}\right] j_{r}^{\prime}\left(k\left(\eta_{0}-\eta\right)\right) j_{r}^{\prime}\left(k\left(\eta_{0}-\eta^{\prime}\right)\right)\right\},
\end{aligned}
$$

where the argument of the Legendre polynomials, $\mathbf{n} \cdot \mathbf{n}^{\prime}$, has been suppressed. Using the relations

$$
j_{r}^{\prime}=-\frac{r+1}{2 r+1} j_{r+1}+\frac{r}{2 r+1} j_{r-1}
$$




\section{R. DURRER}

for Bessel functions, and its iteration for $j^{\prime \prime}$, we can rewrite Eq. (A.12) in terms of the Bessel functions $j_{r-2}$ to $j_{r+2}$.

We now insert the definition of $C_{\ell}$ :

$$
\left\langle\frac{\Delta T}{T}(\mathbf{n}) \cdot \frac{\Delta T}{T}\left(\mathbf{n}^{\prime}\right)\right\rangle_{\left(\mathbf{n} \cdot \mathbf{n}^{\prime}\right)=\cos \theta}=\frac{1}{4 \pi} \Sigma_{\ell}(2 \ell+1) C_{\ell} P_{\ell}(\cos \theta),
$$

and compare the coefficients in Eqs. (A.12) and (A.14). We obtain the somewhat lengthy expression

$$
\begin{aligned}
C_{\ell} & =\frac{2}{\pi} \int d k k^{2} \int d \eta d \eta^{\prime} \dot{H}(k, \eta) \dot{H}^{*}\left(k, \eta^{\prime}\right)\left\{j_{l}\left(k\left(\eta_{0}-\eta\right)\right) j_{l}\left(k\left(\eta_{0}-\eta^{\prime}\right)\right)\right. \\
& \times\left(\frac{1}{(2 \ell-1)(2 \ell+3)}+\frac{2\left(2 \ell^{2}+2 \ell-1\right)}{(2 \ell-1)(2 \ell+3)}+\frac{\left(2 \ell^{2}+2 \ell-1\right)^{2}}{(2 \ell-1)^{2}(2 \ell+3)^{2}}-\frac{4 \ell^{3}}{(2 \ell-1)^{2}(2 \ell+1)}-\frac{4(\ell+1)^{3}}{(2 \ell+1)(2 \ell+3)^{2}}\right) \\
& -\left[j_{\ell}\left(k\left(\eta_{0}-\eta\right)\right) j_{\ell+2}\left(k\left(\eta_{0}-\eta^{\prime}\right)\right)+j_{\ell+2}\left(k\left(\eta_{0}-\eta\right)\right) j_{\ell}\left(k\left(\eta_{0}-\eta^{\prime}\right)\right)\right] \\
& \times \frac{1}{2 l+1}\left(\frac{2(\ell+2)(\ell+1)\left(2 \ell^{2}+2 \ell-1\right)}{(2 \ell-1)(2 \ell+3)^{2}}+\frac{2(\ell+1)(\ell+2)}{(2 \ell+3)}-\frac{8(\ell+1)^{2}(\ell+2)}{(2 \ell+3)^{2}}\right) \\
& -\left[j_{\ell}\left(k\left(\eta_{0}-\eta\right)\right) j_{\ell-2}\left(k\left(\eta_{0}-\eta^{\prime}\right)\right)+j_{\ell-2}\left(k\left(\eta_{0}-\eta\right)\right) j_{\ell}\left(k\left(\eta_{0}-\eta^{\prime}\right)\right)\right] \\
& \times \frac{1}{2 l+1}\left(\frac{2 \ell(\ell-1)\left(2 \ell^{2}+2 \ell-1\right)}{(2 \ell-1)^{2}(2 \ell+3)}+\frac{2 \ell(\ell-1)}{(2 \ell-1)(2}-\frac{8 \ell^{2}(\ell-1)}{(2 \ell-1)^{2}}\right) \\
& +j_{\ell+2}\left(k\left(\eta_{0}-\eta\right)\right) j_{\ell+2}\left(k\left(\eta_{0}-\eta^{\prime}\right)\right)\left(\frac{2(\ell+2)(\ell+1)}{(2 \ell+1)(2 \ell+3)}-\frac{4(\ell+1)(\ell+2)^{2}}{(2 \ell+1)(2 \ell+3)^{2}}+\frac{(\ell+1)^{2}(\ell+2)^{2}}{(2 \ell+1)^{2}(2 \ell+3)^{2}}\right) \\
& \left.+j_{\ell-2}\left(k\left(\eta_{0}-\eta\right)\right) j_{\ell-2}\left(k\left(\eta_{0}-\eta^{\prime}\right)\right)\left(\frac{2 \ell(\ell-1)}{(2 \ell-1)(2 \ell+1)}-\frac{4 \ell(\ell-1)^{2}}{(2 \ell-1)^{2}(2 \ell+1)}+\frac{\ell^{2}(\ell-1)^{2}}{(2 \ell-1)^{2}(2 \ell+1)^{2}}\right)\right\}
\end{aligned}
$$

An analysis of the coefficient of each term reveals that the curly bracket in this expression is just

$$
\{\cdots\}=\ell(\ell-1)(\ell+1)(\ell+2)\left(\frac{j_{\ell}\left(k\left(\eta_{0}-\eta\right)\right)}{\left(k\left(\eta_{0}-\eta\right)\right)^{2}}\right)^{2}
$$

and the result is equivalent to

$$
C_{\ell}=\frac{2}{\pi} \int d k k^{2}|I(\ell, k)|^{2} \ell(\ell-1)(\ell+1)(\ell+2),
$$

with

$$
I(\ell, k)=\int_{\eta_{d e c}}^{\eta_{0}} d \eta \dot{H}(\eta, k) \frac{j_{\ell}\left(\left(k\left(\eta_{0}-\eta\right)\right)\right.}{\left(k\left(\eta_{0}-\eta\right)\right)^{2}} .
$$

\section{APPENDIX B: BOLTZMANN EQUATION AND POLARIZATION}

In this appendix we derive the Boltzmann equation taking into account polarization, and we write it as a hierarchy of equations using an orthonormal expansion in the space of photon directions. Up to the collision term, the Eqs. (4.64), (4.66) and (4.67) are still valid. We first re-derive the collision term taking into account the polarization dependence of Thomson scattering.

Just before the process of recombination during which the fluid description of radiation breaks down, the temperature is $\sim 0.4 \mathrm{eV}$. The electrons and nuclei are nonrelativistic and the dominant collision process is nonrelativistic Thomson scattering.
Thomson scattering depends on the polarization of the incoming radiation field. We describe the polarization state of the radiation field by the Stokes parameters $[22,24,25,23]$ :

For a harmonic electro-magnetic wave with electric field

$$
\mathbf{E}(\mathbf{x}, t)=\left(\boldsymbol{\epsilon}_{1} E_{1}+\boldsymbol{\epsilon}_{2} E_{2}\right) e^{i p \mathbf{n} \cdot \mathbf{x}-i \omega t}
$$

where $\mathbf{n}, \boldsymbol{\epsilon}_{1}$ and $\boldsymbol{\epsilon}_{2}$ form an orthonormal basis and the complex field amplitudes are parameterized as $E_{j}=$ $a_{j} e^{i \delta_{j}}$, the Stokes parameters are given by

$$
\begin{aligned}
& I=a_{1}^{2}+a_{2}^{2}, \\
& Q=a_{1}^{2}-a_{2}^{2}, \\
& U=2 a_{1} a_{2} \cos \left(\delta_{2}-\delta_{1}\right), \\
& V=2 a_{1} a_{2} \sin \left(\delta_{2}-\delta_{1}\right) .
\end{aligned}
$$

$I$ is the intensity of the wave (whose perturbation $\mathcal{M}$ has interested us so far), while $Q$ is a measure of the strength of linear polarization (the ratio of the principal axis of the polarization ellipse). $U$ and $V$ give phase information (the orientation of the ellipse). For nonrelativistic Thomson scattering $V$ is completely decoupled and (since it vanishes at early times) is therefore never generated.

As $Q$ and $U$ vanish in the background, perturbations cannot couple to them (since such terms are 2nd order), and the Liouville equations for $Q$ and $U$ become (neglecting scattering and spatial curvature)

$$
\partial_{\eta}(Q ; U)+i n^{\ell} k_{\ell}(Q ; U)=0 .
$$


The differential cross section of Thomson scattering for a photon with incident polarization $\epsilon_{(i)}$ scattering into the outgoing polarization $\boldsymbol{\epsilon}_{(s)} \equiv \boldsymbol{\epsilon}^{\prime}$ is [22]

$$
\frac{d \sigma}{d \Omega}=\frac{3}{8 \pi} \sigma_{T}\left|\epsilon_{(s)}^{*} \epsilon_{(i)}\right|^{2}
$$

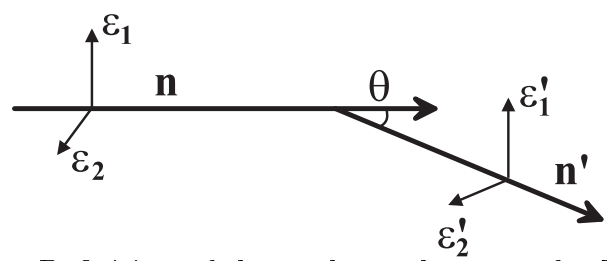

Fig. 10. Definition of the angles and vectors for Thomson scattering in the $\left(\mathbf{n}, \boldsymbol{\epsilon}_{2}\right)$ plane.



Fig. 11. Definition of the angles and vectors for Thomson scattering in the general case. The polarization vectors are oriented like in figure 10.

It is often convenient to introduce the two 'partial' intensities $I_{1} \equiv a_{1}^{2}=(I+Q) / 2$ and $I_{2} \equiv a_{2}^{2}=(I-Q) / 2$. A wave scattered in the $\left(\mathbf{n}, \boldsymbol{\epsilon}_{2}\right)$ plane (see figure 10) by an angle $\theta$ has the intensities

$$
\begin{aligned}
& I_{1}^{(s)}=\frac{3 \sigma_{T}}{8 \pi} I_{1}^{(i)}, \\
& I_{2}^{(s)}=\frac{3 \sigma_{T}}{8 \pi} I_{2}^{(i)} \cos ^{2} \theta,
\end{aligned}
$$

or, expressed in terms of the Stokes parameters,

$$
\left(\begin{array}{c}
I^{(s)} \\
Q^{(s)}
\end{array}\right)=\frac{3 \sigma_{T}}{16 \pi}\left(\begin{array}{cc}
1+\cos ^{2} \theta & \sin ^{2} \theta \\
\sin ^{2} \theta & 1+\cos ^{2} \theta
\end{array}\right)\left(\begin{array}{c}
I^{(i)} \\
Q^{(i)}
\end{array}\right) .
$$

A rotation in the $\left(\boldsymbol{\epsilon}_{1}, \boldsymbol{\epsilon}_{2}\right)$ plane doesn't change the intensity of the wave, but it changes $Q$ and $U$ to

$$
\begin{aligned}
& Q^{\prime}=Q \cos (2 \phi)+U \sin (2 \phi), \\
& U^{\prime}=-U \sin (2 \phi)+Q \cos (2 \phi) .
\end{aligned}
$$

To determine the cross section that a given 'initial' wave $\left(I^{(i)}, Q^{(i)}, U^{(i)}\right)$ propagating in direction $\mathbf{n}$ be scattered into a wave $\left(I^{(s)}, Q^{(s)}, U^{(s)}\right)$ with direction $\mathbf{n}^{\prime}$, we need to go through the following steps (we will use the plane $(\boldsymbol{y}, \boldsymbol{z})$ as reference plane, see figure (11) for definitions of the angles and vectors):

1. Rotate around $\mathbf{n}$ such that the plane $\left(\mathbf{n}, \mathbf{n}^{\prime}\right)$ turns into the plane $(\mathbf{n} \boldsymbol{z})$. One needs to apply the rotation (B.10,B.11) for $\phi=\alpha$ to the Stokes parameters.

2. Rotate the new plane $\left(\mathbf{n}, \mathbf{n}^{\prime}\right)$ around $\boldsymbol{z}$ into the reference plane $(\boldsymbol{y}, \boldsymbol{z})$. This operation does not influence the incoming Stokes parameters.

3. Now we are in the known case of (B.8) and (B.9). Hence we can apply the scattering matrix.

4. We then rotate the scattering plane back around $\boldsymbol{z}$ into the old $\left(\boldsymbol{z}, \mathbf{n}^{\prime}\right)$ plane. This does not change the scattered Stokes parameters.

5. Finally we rotate around $\mathbf{n}^{\prime}$ by the angle $\alpha^{\prime}$ to reach the original state. To do this, we have to apply the rotation matrix (B.10,B.11) again, but now for $\phi=\alpha^{\prime}$.

Following the steps outlined above, we recover the scattering matrix in the basis $\left(I_{1}, I_{2}, U\right)$ given in equations (B.13)-(B.16) (see also [23]). $V$ is completely decoupled from the other parameters and follows an evolution which is independent of the rest. Hence by starting with $V\left(t \ll t_{\mathrm{dec}}\right)=0$ it will stay zero and can be neglected. The scattering matrix $P$, which determines the (non vanishing) scattered Stokes parameters from the initial ones,

$$
\left(\begin{array}{c}
I_{1}^{(s)} \\
I_{2}^{(s)} \\
U^{(s)}
\end{array}\right)=\frac{\sigma_{T}}{4 \pi} P\left(\begin{array}{c}
I_{1}^{(i)} \\
I_{2}^{(i)} \\
U^{(i)}
\end{array}\right)
$$

is then given by

$$
P=\left[P^{(0)}+\sqrt{1-\mu^{2}} \sqrt{1-\mu^{2}} P^{(1)}+P^{(2)}\right],
$$

where

$$
P^{(0)}=\frac{3}{4}\left(\begin{array}{ccc}
2\left(1-\mu^{2}\right)\left(1-\mu^{\prime 2}\right)+\mu^{2} \mu^{\prime 2} & \mu^{2} & 0 \\
\mu^{\prime 2} & 1 & 0 \\
0 & 0 & 0
\end{array}\right),
$$




\section{R. DURRER}

$$
\begin{gathered}
P^{(1)}=\frac{3}{4}\left(\begin{array}{ccc}
4 \mu \mu^{\prime} \cos \left(\phi^{\prime}-\phi\right) & 0 & 2 \mu \sin \left(\phi^{\prime}-\phi\right) \\
0 & 0 & 0 \\
-4 \mu^{\prime} \sin \left(\phi^{\prime}-\phi\right) & 0 & 2 \cos \left(\phi^{\prime}-\phi\right)
\end{array}\right), \\
P^{(2)}=\frac{3}{4}\left(\begin{array}{ccc}
\mu^{2} \mu^{2} \cos \left[2\left(\phi^{\prime}-\phi\right)\right] & -\mu^{2} \cos \left[2\left(\phi^{\prime}-\phi\right)\right] & \mu^{2} \mu^{\prime} \sin \left[2\left(\phi^{\prime}-\phi\right)\right] \\
-\mu^{\prime 2} \cos \left[2\left(\phi^{\prime}-\phi\right)\right] & \cos \left[2\left(\phi^{\prime}-\phi\right)\right] & -\mu^{\prime} \sin \left[2\left(\phi^{\prime}-\phi\right)\right] \\
-2 \mu \mu^{\prime 2} \sin \left[2\left(\phi^{\prime}-\phi\right)\right] & 2 \mu \sin \left[2\left(\phi^{\prime}-\phi\right)\right] & 2 \mu \mu^{\prime} \cos \left[2\left(\phi^{\prime}-\phi\right)\right]
\end{array}\right) .
\end{gathered}
$$

As we are in an isotropic situation, we will perform all the calculations in a special coordinate system with $\mathbf{k} \| \hat{z}$ and $\mathbf{n}, \mathbf{n}^{\prime}$ as in Fig. 11. Clearly the results are independent of this coordinate choice.

The matrix $R$ connecting $\left(I_{1}, I_{2}, U\right)$ with $(I, Q, U)$ is given by

$$
\left(\begin{array}{l}
I_{1} \\
I_{2} \\
U
\end{array}\right)=\left(\begin{array}{c}
1 / 2(I+Q) \\
1 / 2(I-Q) \\
U
\end{array}\right)=\frac{1}{2}\left(\begin{array}{rrr}
1 & 1 & 0 \\
1 & -1 & 0 \\
0 & 0 & 2
\end{array}\right)\left(\begin{array}{c}
I \\
Q \\
U
\end{array}\right) \equiv R\left(\begin{array}{c}
I \\
Q \\
U
\end{array}\right)
$$

To calculate the collision term including polarization, we change into the $\left(I_{1}, I_{2}\right)$ basis. For each of the two intensities $\lambda \in\{1,2\}$ we then have the collision term given by

$$
C\left[f^{(\lambda)}\right]=\frac{d f_{+}^{(\lambda)}}{d \eta}-\frac{d f_{-}^{(\lambda)}}{d \eta}
$$

where $f_{+}^{(\lambda)}$ and $f_{-}^{(\lambda)}$ denote the distribution of photons in the polarization state $\lambda$ scattered into respectively out of the beam due to Compton scattering.

In the matter (baryon/electron) rest frame, which we indicate by a prime, we know that

$$
\frac{d f_{+}^{(\lambda) \prime}}{d t^{\prime}}(p, \mathbf{n})=\frac{\sigma_{T} n_{e}}{4 \pi} \int f^{(\delta) \prime}\left(p^{\prime}, \mathbf{n}^{\prime}\right) P_{\delta}^{\lambda}\left(\mathbf{n}, \mathbf{n}^{\prime}\right) d \Omega^{\prime},
$$

where $n_{e}$ denotes the electron number density and $P_{\delta}^{\lambda}$ is the $2 \times 2$ upper left corner of the normalized Thomson scattering matrix (B.13). In the baryon rest frame which moves with four velocity $u$, the photon energy is given by

$$
p^{\prime}=p_{\mu} u^{\mu}
$$

We denote the photon energy with respect to a tetrad adapted to the slicing of space-time into $\{\eta=$ constant $\}$ hyper-surfaces by $p$ :

$$
p=p_{\mu} n^{\mu}, \quad \text { with } \quad n=a^{-1}\left[(1-A) \partial_{\eta}+B^{i} \partial_{i}\right]
$$

The lapse function and the shift vector of the slicing are given by $\alpha=a(1+A)$ and $\boldsymbol{\beta}=-B^{i} \partial_{i}$. In first order,

$$
p_{0}=a p(1+A)-a p n_{i} B^{i}
$$

and to zeroth order $p_{i}=a p n_{i}$. Furthermore, the baryon four-velocity has the form $u^{0}=a^{-1}(1-A), \quad u^{i}=u^{0} v^{i}$ up to first order. This yields

$$
p^{\prime}=p_{\mu} u^{\mu}=p\left(1+n_{i}\left(v^{i}-B^{i}\right)\right)
$$

Using this identity and performing the integration over photon energies, we obtain

$$
\rho_{\gamma} \frac{d \iota_{+}^{(\lambda)}(\mathbf{n})}{d \eta^{\prime}}=a \rho_{\gamma} \sigma_{T} n_{e}\left[1+4 n_{i}\left(v^{i}-B^{i}\right)+\frac{1}{4 \pi} \int \iota^{(\delta)}\left(\mathbf{n}^{\prime}\right) P_{\delta}^{\lambda}\left(\mathbf{n}, \mathbf{n}^{\prime}\right) d \Omega^{\prime}\right]
$$

Photons which are scattered leave the beam, with the probability given by the Thomson cross section (see, e.g., [26])

$$
\frac{d f_{-}^{(\lambda)}}{d t^{\prime}}=\sigma_{T} n_{e} f^{(\lambda) \prime}\left(p^{\prime}, \mathbf{n}\right)
$$

so that we finally have 


$$
C^{(\lambda) \prime}=\frac{4 \pi}{\rho_{\gamma} a^{4}} \int d p\left(\frac{d f_{+}^{(\lambda)}}{d t^{\prime}}-\frac{d f_{-}^{(\lambda)}}{d t^{\prime}}\right) p^{3}=\frac{1}{2} \sigma_{T} n_{e}\left[4 n_{i}\left(v^{i}-B^{i}\right)-\iota^{(\lambda)}+\frac{1}{4 \pi} \int \iota^{(\delta)}\left(\mathbf{n}^{\prime}\right) P_{\delta}^{\lambda}\left(\mathbf{n}, \mathbf{n}^{\prime}\right) d \Omega^{\prime}\right]
$$

By setting $C^{(\mathcal{M})}=C^{(1)}+C^{(2)}$ and $C^{(Q)}=C^{(1)}-C^{(2)}$ we transform the collision integral back to the normal Stokes parameters. The term for $U$ has the same form as the one for $Q$, just with the corresponding matrix elements in the integral. The Boltzmann equation then finally becomes (setting $\mathcal{E} \equiv(\mathcal{M}, Q, U)$ and for the flat case, $\kappa=0)$ :

$$
\begin{gathered}
\dot{\mathcal{M}}+i \mu k \mathcal{M}=4 i \mu k\left(\Phi-\Psi+n^{m} \Sigma_{m}^{(V)}\right)+4 n^{\ell} n^{m} \dot{H}_{m \ell}+a \sigma_{T} n_{e}\left[-\mathcal{M}-4 i \mu V_{b}+4 n^{\ell} \omega_{b, \ell}+\int d \Omega^{\prime} P_{1}^{\alpha} \mathcal{E}_{\alpha}^{\prime}\right], \\
\dot{Q}+i \mu k Q=a \sigma_{T} n_{e}\left[-Q+\int d \Omega^{\prime} P_{2}^{\alpha} \mathcal{E}_{\alpha}^{\prime}\right], \\
\dot{U}+i \mu k U=a \sigma_{T} n_{e}\left[-U+\int d \Omega^{\prime} P_{3}^{\alpha} \mathcal{E}_{\alpha}^{\prime}\right],
\end{gathered}
$$

where we have to use the scattering matrix transformed into the $(\mathcal{M}, Q, U)$ basis,

$$
\begin{aligned}
& P=P_{S}+P_{V}+P_{T} \\
& P_{S}=R^{-1} P^{(0)} R=\frac{3}{8}\left(\begin{array}{ccc}
3-\mu^{2}-\mu^{\prime 2}+3 \mu^{2} \mu^{2} & \left(1-3 \mu^{2}\right)\left(1-\mu^{\prime 2}\right) & 0 \\
\left(1-\mu^{2}\right)\left(1-3 \mu^{\prime 2}\right) & 3\left(1-\mu^{2}\right)\left(1-\mu^{\prime 2}\right) & 0 \\
0 & 0 & 0
\end{array}\right), \\
& P_{V}=\sqrt{1-\mu^{2}} \sqrt{1-\mu^{\prime 2}} R^{-1} P^{(1)} R=\frac{3}{2} \sqrt{1-\mu^{2}} \sqrt{1-\mu^{\prime 2}}\left(\begin{array}{ccc}
\mu \mu^{\prime} C & \mu \mu^{\prime} C & -\mu S \\
\mu \mu^{\prime} C & \mu \mu^{\prime} C & -\mu S \\
\mu^{\prime} S & \mu^{\prime} S & C
\end{array}\right), \\
& P_{T}=R^{-1} P^{(2)} R=\frac{3}{8}\left(\begin{array}{ccc}
\left(1-\mu^{2}\right)\left(1-\mu^{\prime 2}\right) C_{T} & -\left(1-\mu^{2}\right)\left(1+\mu^{\prime 2}\right) C_{T} & 2\left(1-\mu^{2}\right) \mu^{\prime} S_{T} \\
-\left(1+\mu^{2}\right)\left(1-\mu^{\prime 2}\right) C_{T} & \left(1+\mu^{2}\right)\left(1+\mu^{\prime 2}\right) C_{T} & -2\left(1+\mu^{2}\right) \mu^{\prime} S_{T} \\
-2 \mu\left(1-\mu^{\prime 2}\right) S_{T} & 2 \mu\left(1+\mu^{\prime 2}\right) S_{T} & 4 \mu \mu^{\prime} C_{T}
\end{array}\right)
\end{aligned}
$$

with $C=\cos \left(\phi-\phi^{\prime}\right), S=\sin \left(\phi-\phi^{\prime}\right)$ and $C_{T}=\cos \left(2\left(\phi-\phi^{\prime}\right)\right), S_{T}=\sin \left(2\left(\phi-\phi^{\prime}\right)\right)$. The parts $P_{S}, P_{V}, P_{T}$ of $P$ describe the scattering of the scalar, vector and tensor contribution to $\mathcal{E}$ respectively.

The functions $\mathcal{M}, Q$ and $U$ depend on the wave vector $\mathbf{k}$, the photon direction $\mathbf{n}$ and conformal time $\eta$. We choose for each $\mathbf{k}$-mode a reference system with $z$-axis parallel to $\mathbf{k}$. For scalar perturbations we achieve in this way azimuthal symmetry — the right-hand side of the Boltzmann equation and therefore also the brightness $\mathcal{M}^{(S)}$ depend only on $\mu=(\hat{\mathbf{k}} \cdot \mathbf{n})$ and can be expanded in Legendre polynomials. The right-hand side of the Boltzmann equation also determines the azimuthal dependence of vector and tensor perturbations. One can continue with this approach, but the resulting equations for $Q$ and $U$ and especially also their power spectra depend explicitly on the coordinate system. Therefore, we prefer an approach which is inherently covariant under rotation.

\section{A. Electric and magnetic polarization}

The Stokes parameters explicitly depend on the coordinate system, and Eqs. (B.21) and (B.22) transform rather complicated under rotations of the coordinate system. A method to characterize CMB polarization due to nonrelativistic Thomson scattering which is more convenient than the Stokes parameters since its transformation properties are very simple has been developed some years ago [51-53,55,56]. A detailed derivation of this method goes beyond the scope of this report. Here we just repeat the definitions and the main results. We set

$$
\mathcal{T}=(\mathcal{M}, Q+i U, Q-i U)
$$

In terms of this vector the collision integral above can we written (in vector form) as

$$
\boldsymbol{C}[\mathcal{T}]=a \sigma_{T} n_{e}\left[-\boldsymbol{T}+\left(\frac{1}{4 \pi} \int \mathrm{d} \Omega^{\prime} \mathcal{M}^{\prime}+\left(\mathbf{n} \cdot \mathbf{v}_{b}\right), 0,0\right)+\frac{1}{10} \sum_{m=-2}^{2} \int \mathrm{d} \Omega^{\prime} P^{(m)}\left(\mathbf{n}, \mathbf{n}^{\prime}\right) \mathcal{T}^{\prime}\right]
$$

From Eqs. (B.13) to (B.17) one can determine the scattering matrix for the vector $\mathcal{T}$ 


\section{R. DURRER}

$$
P^{(m)}=\left(\begin{array}{ccc}
Y_{2}^{m \prime} Y_{2}^{m} & -\sqrt{\frac{3}{2}}{ }_{2} Y_{2}^{m \prime} Y_{2}^{m} & -\sqrt{\frac{3}{2}}-2 Y_{2}^{m \prime} Y_{2}^{m} \\
-\sqrt{6} Y_{2}^{m \prime}{ }_{2} Y_{2}^{m} & 3{ }_{2} Y_{2}^{m \prime}{ }_{2} Y_{2}^{m} & 3{ }_{-2} Y_{2}^{m \prime}{ }_{2} Y_{2}^{m} \\
-\sqrt{6} Y_{2}^{m \prime}{ }_{-2} Y_{2}^{m} & 3{ }_{2} Y_{2}^{m \prime}{ }_{-2} Y_{2}^{m} & 3{ }_{-2} Y_{2}^{m \prime}{ }_{2} Y_{2}^{m}
\end{array}\right)
$$

where ${ }_{s} Y_{l}^{m \prime}={ }_{s} Y_{l}^{m *}\left(n^{\prime}\right)$ and ${ }_{s} Y_{l}^{m}$ are the spin-weighted spherical harmonics [54,55].

We now decompose the Fourier components of the temperature anisotropy $\mathcal{M}$ and the polarization variables $E$ and $B$ as

$$
\begin{aligned}
& \mathcal{M}=\sum_{\ell} \sum_{m=-2}^{2} \mathcal{M}_{\ell}^{(m)}{ }_{0} G_{\ell}^{m} \\
& Q \pm i U=\sum_{\ell} \sum_{m=-2}^{2}\left(E_{\ell}^{(m)} \pm i B_{\ell}^{(m)}\right)_{2} G_{\ell}^{m}(\mathbf{n})
\end{aligned}
$$

Here $m=0$ is the scalar mode, $m= \pm 1$ are the vector and $m= \pm 2$ are the tensor modes. The functions ${ }_{s} G_{\ell}^{m}$ are closely related to the spin weighted harmonics ${ }_{s} Y_{\ell}^{m}$ :

$$
{ }_{s} G_{\ell}^{m}(\mathbf{n})=(-i)^{\ell} \sqrt{\frac{4 \pi}{2 \ell+1}}{ }_{s} Y_{\ell}^{m}(\mathbf{n})
$$

The evolution equations in term of these variables can be given in the following compact form [56]

$$
\begin{aligned}
& \dot{\mathcal{M}}_{\ell}^{(m)}-k\left[\frac{{ }_{0} \kappa_{\ell}^{m}}{2 \ell-1} \mathcal{M}_{\ell-1}^{(m)}-\frac{0 \kappa_{\ell+1}^{m}}{2 \ell+3} \mathcal{M}_{\ell+1}^{(m)}\right]=-n_{e} \sigma_{T} a \mathcal{M}_{\ell}^{(m)}+S_{\ell}^{(m)}, \quad(\ell \geq m), \\
& \dot{E}_{\ell}^{(m)}-k\left[\frac{2 \kappa_{\ell}^{m}}{2 \ell-1} E_{\ell-1}^{(m)}-\frac{2 m}{\ell(\ell+1)} B_{\ell}^{(m)}-\frac{2 \kappa_{\ell+1}^{m}}{2 \ell+3} E_{\ell+1}^{(m)}\right]=-n_{e} \sigma_{T} a\left[E_{\ell}^{(m)}+\sqrt{6} C^{(m)} \delta_{\ell, 2},\right. \\
& \dot{B}_{\ell}^{(m)}-k\left[\frac{{ }_{2} \kappa_{\ell}^{m}}{2 \ell-1} B_{\ell-1}^{(m)}+\frac{2 m}{\ell(\ell+1)} E_{\ell}^{(m)}-\frac{{ }_{2} \kappa_{\ell+1}^{m}}{2 \ell+3} B_{\ell+1}^{(m)}\right]=-n_{e} \sigma_{T} a B_{\ell}^{(m)},
\end{aligned}
$$

where we have set

$$
\begin{array}{lll}
S_{0}^{(0)}=n_{e} \sigma_{T} a \mathcal{M}_{0}^{(0)}, & S_{1}^{(0)}=n_{e} \sigma_{T} a 4 V_{b}+4 k(\Psi-\Phi), & S_{2}^{(0)}=n_{e} \sigma_{T} a C^{(0)} \\
S_{1}^{(1)}=n_{e} \sigma_{T} a 4 \omega_{b}, & S_{2}^{(1)}=n_{e} \sigma_{T} a C^{(1)}+4 k \Sigma, & S_{2}^{(2)}=n_{e} \sigma_{T} a C^{(2)}+4 \dot{H}
\end{array}
$$

and $C^{(m)}=\frac{1}{10}\left[\mathcal{M}_{2}^{(m)}-\sqrt{6} E_{2}^{(m)}\right]$. The coupling coefficients are

$$
{ }_{s} \kappa_{\ell}^{m}=\sqrt{\frac{\left(\ell^{2}-m^{2}\right)\left(\ell^{2}-s^{2}\right)}{\ell^{2}}} .
$$

Note that for scalar perturbations, $m=0, B$-polarization is not sourced and we have $B_{\ell}^{(0)} \equiv 0$.

Finally we want to connect the intensities $\mathcal{M}_{\ell}^{(m)}$ with the more familiar expansion of the scalar $(S)$, vector $(V)$ and tensor $(T)$ contributions to the brightness function in terms of Legendre polynomials. Usually one sets

$$
\mathcal{M}=\mathcal{M}^{(S)}+\mathcal{M}^{(V)}+\mathcal{M}^{(T)} .
$$

Here $\mathcal{M}^{(S)}$ only depends on $\mu=(\mathbf{n} \cdot \mathbf{k}) / k$ and the $\mathbf{n}$-dependence of $\mathcal{M}^{(V)}$ and $\mathcal{M}^{(T)}$ can be written as

$$
\begin{aligned}
& \mathcal{M}^{(V)}(\mu, \phi)=\sqrt{1-\mu^{2}}\left[\mathcal{M}_{1}^{(V)}(\mu) \cos \phi+\mathcal{M}_{2}^{(V)}(\mu) \sin \phi\right], \\
& \mathcal{M}^{(T)}(\mu, \phi)=\left(1-\mu^{2}\right)\left[\mathcal{M}_{+}^{(T)} \cos (2 \phi)+\mathcal{M}_{\times}^{(T)} \sin (2 \phi)\right],
\end{aligned}
$$

where $\phi$ is the azimuthal angle in the plane normal to $\mathbf{k}$. Each of the functions $\mathcal{M}_{\bullet}^{(S, V, T)}(\mu)$ is now expanded in Legendre polynomials

$$
\mathcal{M}_{\bullet}^{(S, V, T)}=\sum_{\ell}(-i)^{\ell}(2 \ell+1) \sigma_{\bullet, \ell}^{(S, V, T)} P_{\ell}(\mu)
$$

The coefficients $\sigma_{\bullet}^{(S, V, T)}$ are then related to $\mathcal{M}_{\ell}^{(m)}$ via the identities 


$$
\begin{aligned}
& \mathcal{M}_{\ell}^{(0)}=(2 \ell+1) \sigma_{\ell}^{(S)}, \\
& \mathcal{M}_{\ell}^{( \pm 1)}=\sqrt{\ell(\ell+1)}\left[\sigma_{2, \ell+1}^{(V)} \pm i \sigma_{1, \ell+1}^{(V)}+\sigma_{1, \ell-1}^{(V)} \pm i \sigma_{1, \ell-1}^{(V)}\right], \\
& \mathcal{M}_{\ell}^{( \pm 2)}=-\sqrt{\frac{(\ell+2) !}{(\ell-2) !}}\left[\frac{1}{2 \ell+3} \sigma_{\uparrow \downarrow, \ell+2}^{(T)}+\frac{2(2 \ell+1)}{(2 \ell-1)(2 \ell+3)} \sigma_{\uparrow \downarrow, \ell}^{(T)}+\frac{1}{2 \ell-1} \sigma_{\uparrow \downarrow, \ell-2}^{(T)}\right],
\end{aligned}
$$

where

$$
\sigma_{\uparrow \downarrow, \ell}=\sigma_{+\ell} \mp i \sigma_{\times \ell}
$$

We do not repeat this correspondence for the Stokes parameters $Q$ and $U$ since it is rather complicated and not very useful as it depends on the coordinate system chosen.

\section{B. Power spectra}

In the previous appendix and in Section 4 we have derived the expression for the CMB anisotropy power spectrum for scalar and tensor perturbations. Here we give the general expression for scalar, vector and tensor fluctuations, polarizations and cross correlations. To make contact with the results derived before, one has to use Eqs. (B.37,B.38) and (B.40) and neglect the collision term in the Boltzmann equation.

We expand the present CMB anisotropies and polarization in spherical harmonics: $\Delta T\left(\mathbf{n}, \eta_{0}\right) / T_{0}=$ $\sum_{\ell m} a_{\ell m} Y_{\ell}^{m}(\mathbf{n})$. The coefficients $a_{\ell m}$ are random variables with zero mean and rotationally invariant variances, $C_{\ell} \equiv\left\langle\left|a_{\ell m}\right|^{2}\right\rangle$. The correlation function of the anisotropy pattern then has the standard expression:

$$
\left\langle\frac{\delta T}{T_{0}}\left(\mathbf{n}_{1}\right) \frac{\delta T}{T_{0}}\left(\mathbf{n}_{2}\right)\right\rangle=\frac{1}{4 \pi} \sum_{\ell}(2 \ell+1) C_{\ell} P_{\ell}(\cos \theta),
$$

where $\cos \theta=\mathbf{n}_{1} \cdot \mathbf{n}_{2}$ and $\langle\cdots\rangle$ denotes ensemble average. We use the Fourier transform normalization

$$
\hat{f}(\mathbf{k})=\frac{1}{V} \int f(\mathbf{x}) \exp (i \mathbf{k} \cdot \mathbf{x}) d^{3} x
$$

with some normalization volume $V$. Using statistical homogeneity we have

$$
\left\langle\frac{\delta T}{T_{0}}\left(\mathbf{n}_{1}\right) \frac{\delta T}{T_{0}}\left(\mathbf{n}_{2}\right)\right\rangle=\frac{1}{V} \int d^{3} x\left\langle\frac{\delta T}{T_{0}}\left(\mathbf{x}, \mathbf{n}_{1}\right) \frac{\delta T}{T_{0}}\left(\mathbf{x}, \mathbf{n}_{2}\right)\right\rangle=\frac{1}{(2 \pi)^{3}} \int d^{3} k\left\langle\frac{\delta T}{T_{0}}\left(\mathbf{k}, \mathbf{n}_{1}\right) \frac{\delta T}{T_{0}}\left(\mathbf{k}, \mathbf{n}_{2}\right)\right\rangle .
$$

Inserting our ansatz (B.37) for $\frac{\delta T}{T_{0}}=\frac{1}{4} \mathcal{M}$, and using the addition theorem for spherical harmonics, $P_{\ell}\left(\mathbf{n}_{1} \cdot \mathbf{n}_{2}\right)=\frac{4 \pi}{2 \ell+1} \sum_{m} Y_{\ell m}^{*}\left(\mathbf{n}_{1}\right) Y_{\ell m}\left(\mathbf{n}_{2}\right)$, we find

$$
\begin{aligned}
\left\langle\frac{\delta T}{T_{0}}\left(\mathbf{n}_{1}\right) \frac{\delta T}{T_{0}}\left(\mathbf{n}_{2}\right)\right\rangle & =\frac{1}{8 \pi} \sum_{\ell, \ell^{\prime}, m, m^{\prime}}(-1)^{\left(\ell-\ell^{\prime}\right)} Y_{\ell m}\left(n_{1}\right) Y_{\ell^{\prime} m^{\prime}}^{*}\left(n_{2}\right) \int k^{2} d k d \Omega_{\hat{\mathbf{k}}} Y_{\ell m}^{*}(\hat{\mathbf{k}}) Y_{\ell^{\prime} m^{\prime}}(\hat{\mathbf{k}})\left\langle\sigma_{\ell} \sigma_{\ell^{\prime}}^{*}\right\rangle(k) \\
& =\frac{1}{32 \pi^{2}} \sum_{\ell}(2 \ell+1) P_{\ell}\left(\mathbf{n}_{1} \cdot \mathbf{n}_{2}\right) \int k^{2} d k\left\langle\sigma_{\ell} \sigma_{\ell}^{*}\right\rangle(k),
\end{aligned}
$$

from which we conclude

$$
C_{\ell}^{\mathcal{M M},(S)}=\frac{1}{8 \pi} \int k^{2} d k\left\langle\left|\sigma_{\ell}^{(S)}\left(t_{0}, k\right)\right|^{2}\right\rangle
$$

where the superscript $(S)$ indicates that Eq. (B.45) gives the contribution from scalar perturbations and $\mathcal{M M}$ means that it is the contribution to the intensity perturbation.

The $Q Q, U U, \mathcal{M} Q, \mathcal{M} U$ and $Q U$ correlators depend with the Stokes parameters on the particular coordinate system chosen. It is much more convenient to express the polarization power spectra in terms of the variables $E$ and $B$ which are independent of the coordinate system. Furthermore, since $B$ is parity odd, its correlators with $\mathcal{M}$ and $E$ vanishes. One finds the simple general expression [56]

$$
(2 \ell+1)^{2} C_{\ell}^{X Y(m)}=\frac{n_{m}}{8 \pi} \int k^{2} d k X_{\ell}^{(m)} Y_{\ell}^{(m) *}
$$

where $n_{m}=1$ for $m=0$ and $n_{m}=2$ for $m=1,2$, accounting for the number of modes. Here $X$ and $Y$ run over $\mathcal{M}, E$ and $B$. 
[1] M. Bucher, K. Moodley and N. Turok, preprint astroph/0012141 (2000).

[2] R. Trotta, A. Riazuelo and R. Durrer, Phys. Rev. Lett. 87, 231301 (2001).

[3] J. M. Stewart and M. Walker, Proc. R. Soc. London A 341, 49 (1974).

[4] J. Bardeen, Phys. Rev. D 22, 1882 (1980).

[5] H. Kodama, M. Sasaki, Prog. Theor. Phys. Suppl 78, 1 (1984).

[6] R. Durrer, Fund. of Cosmic Physics 15, 209 (1994).

[7] Particle Data Group, Europ. Phys. J. C 3, 128 (1998).

[8] G. F. Smoot et al., Astrophys. J. 396, L1 (1992).

[9] R. R. Caldwell, R. Dave, P. J. Steinhardt, Phys. Rev. Lett. 80, 1582 (1998).

[10] We will use units with $\hbar=c=k_{\mathrm{B}}=1$ throughout this report. The Stefan-Boltzmann constant is then given by $a_{\mathrm{SB}}=\pi^{2} k_{\mathrm{B}}^{4} /\left(60 \hbar^{3} c^{2}\right)=\pi^{2} / 60$.

[11] Cosmic Background Explorer, NASA satellite launched 1990.

[12] $Y_{i j}^{(V)} \sigma^{(V)}$ is the shear of the hyper-surfaces of constant time.

[13] Here $\lambda$ is a typical size of a perturbation. For a given Fourier mode $k$, it is $\lambda \sim \pi / k$.

[14] T. Padmanabhan, Structure formation in the universe, (Cambridge University Press, 1993).

[15] P. J. E. Peebles, Principles of Physical Cosmology, (Princeton University Press, 1993).

[16] E. K. Conklin, Nature 222, 971 (1969).

[17] P. S. Henry, Nature 231, 516 (1971).

[18] M. Bruni, P. Dunsby, G. Ellis, Astrophys. J 395, 34 (1992).

[19] E. M. Lifshitz, Sov. J. Phys. (JETP) 10, 116 (1946).

[20] M. Bucher, K. Moodley, N. Turok, Phys. Rev. D 62 083508 (2000).

[21] P. J. E. Peebles, Large Scale Structure of the Universe (Princeton University Press, 1980).

[22] J. D. Jackson, Electrodynamics, (Wiley \& Sons, New York, 1962, 1975).

[23] S. Chandrasekhar, Radiative Transfer, (Dover Publications, New York, 1960).

[24] A. Kosowsky, Ann. Phys. 246, 49 (1996).

[25] A. Melchiorri, N. Vittorio, in: Proceedings of the NATO Advanced Study Institute 1996, Strasbourg, preprint astro-ph/9610029, (1996).

[26] E. M. Lifshitz, L. P. Pitajewski, Lehrbuch der Theoretischen Physik, Band X (Akademie Verlag Berlin, 1983).

[27] R. K. Sachs, A. M. Wolfe, Astrophys. J. 147, 73 (1967).

[28] A. G. Doroshkevich, Ya. B. Zel'dovich, R. A. Sunyaev, Sov. Astron. 22, 523 (1978).
[29] E. Harrison, Phys. Rev. D 12726 (1970); Ya. B. Zel'dovich, Mont. Not. R. Astron. Soc. 160, P1 (1972).

[30] R. Durrer, A. Gangui, M. Sakellariadou, Phys. Rev. Lett. 76, 579 (1996).

[31] U. Pen, D. Spergel, N. Turok, Phys Rev. D 49, 692 (1994).

[32] R. Durrer, Z. Zhou, Phys. Rev. D 53, 5394 (1996).

[33] B. Allen, R. R. Caldwell, S. Dodelson, L. Knox, E. P. S. Shellard, A. Stebbins, Phys. Rev. Lett. 79, 2624 (1997).

[34] R. Durrer, M. Gasperini, M. Sakellariadou, G. Veneziano, Phys. Rev. D 59, 043511 (1999)

[35] T. Kanazawa, M. Kawasaki, N. Sugiyama, T. Yanagida, Prog. Theor. Phys. 1001055 (1998).

[36] P. J. E. Peebles, Astrophys. J. 510, 531 (1999).

[37] U. Seljak, M. Zaldarriaga, Astrophys. J. 496, 437 (1996).

[38] A. Lewis, A. Challinor, A. Lasenby, Astrophys. J. 538, $473(2000)$.

[39] C. L. Bennett et al., Astrophys. J. Lett. 464, L1 (1996).

[40] C. B. Netterfield et al., preprint astro-ph/0104460 (2001).

[41] A. T. Lee et al., preprint astro-ph/0104459 (2001).

[42] N. W. Halverson et al., preprint astro-ph/0104489 (2001).

[43] P. de Bernardis et al., preprint astro-ph/0105296 (2001).

[44] C. Pryke, N. W. Halverson, E. M. Leitch, J. Kovac, J. E. Carlstrom, W. L. Holzapfel, M. Dragovan, preprint astro-ph/0104490 (2001).

[45] R. Stompor et al., preprint astro-ph/0105062 (2001).

[46] X. Wang, M. Tegmark, M. Zaldarriaga, preprint astroph/0105091 (2001).

[47] M. Abramowitz, I. Stegun, Handbook of Mathematical Functions (Dover Publications, New York, 1972).

[48] W. Hu, N. Sugiyama, Phys. Rev. D 51, 2599 (1995), and references therein.

[49] J. M. Stewart, Non-Equilibrium Relativistic Kinetic Theory, (Springer Lecture Notes in Physics, Vol. 10, edited by J. Ehlers, K. Hepp and H.A. Wienmüller, 1971).

[50] J. P. Uzan, Class. Quantum. Gravit. 15, 1063, (1998).

[51] U. Seljak, M. Zaldarriaga, Phys. Rev. Lett. 78, 2054 (1997).

[52] M. Zaldarriaga, U. Seljak, Phys. Rev. D 55, 1830 (1997).

[53] M. Kamionkowski, A. Kosowsky, A. Stebbins, Phys. Rev. Lett. 782058 (1997).

[54] E. Newman, R. Penrose, J. Math. Phys. 7, 863 (1966).

[55] M. Kamionkowski, A. Kosowsky, A. Stebbins, Phys. Rev. D 55, 7368 (1997).

[56] W. Hu, U. Seljak, M. White, M. Zaldarriaga, Phys. Rev. D 57, 3290 (1998). 


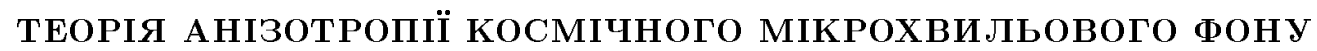

\author{
Р. Дюрер \\ Інститут теоретичнӧ̈ фізики Женевського універитету \\ вул. Е. Ансермет, 24, 1211 Женева 4, ІІбейцаріл; \\ Факультет природничих наук Інституту перспективних досліджень \\ Алея Айнштайна, Прінстон, NJ 08540, CIIIA
}

\begin{abstract}
Зроблено огляд теорії анізотропії космічного мікрохвильового фону, який є оновленою версією курсу лекцій, прочитаних навесні 1999 р. в "troisieme cycle de la Suisse Romande”. Подано вступ „оо калібрувальноінваріантної теорії космологічних збурень і в цьому контексті розглянуто анізотропію мікрохвильового фону. Отримано прості аналітичні апроксимапії положень акустичних піків адіябатичних і ізотермічних збурень. Обговорено загасання Сілка в аналітичному підході. Завершують огляд короткий опис сучасного стану спостережень, оңнки космологічних параметрів та заключне обговорення. У Додатках виведено повну систему диференпійних рівнянь для анізотропії космічного мікрохвильового фону та його поляризапї, яка необхідна в числовому пі,дході.
\end{abstract}

